\title{
A functional analysis of a signaling center of the insect head
}
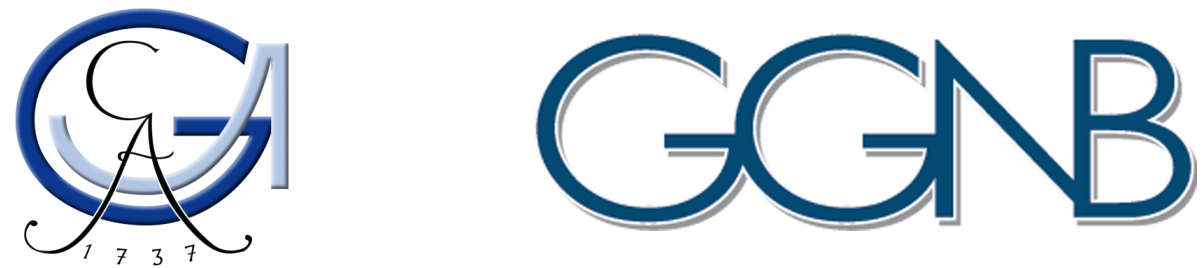

\author{
Georg Oberhofer \\ born in Innichen (Italy)
}

Department of Delopmental Biology

Georg-August-University Göttingen

A thesis submitted for the degree of PhilosophiceDoctor (Ph.D.)

December, 2013 
Member of the Thesis Committee (Reviewer): Prof. Dr. Gregor Bucher Department of Developmental Biology, University Göttingen

Member of the Thesis Committee (Reviewer): Prof. Dr. Andreas Wodarz Department of Anatomy and Cell Biology, Universitätsmedizin Göttingen

Member of the Thesis Committee: Prof. Dr. Steven Johnsen Department of Tumor Biology, Universitätsklinikum Hamburg-Eppendorf

Day of the defense:

Signature from head of $\mathrm{PhD}$ committee: 


\section{Declaration}

I herewith declare that I have produced this thesis without the prohibited assistance of third parties and without making use of aids other than those specified; notions taken over directly or indirectly from other sources have been identified as such. This thesis has not previously been presented in identical or similar form to any other German or foreign examination board.

Göttingen, 31 December 2013 


\begin{abstract}
Most of our knowledge on embryonic development comes from the fruit fly Drosophila melanogaster. An elaborate genetic model explains the segmentation process in the trunk. However, the anterior head region is patterned in a different way, and many details are not well understood so far. This work analyzes the genetic regulations that govern anterior head patterning in the the red flour beetle Tribolium castaneum.

Especially, functional analysis with RNAi against crucial components of the Wnt and hedgehog signaling pathways is used to reveal the genetic interactions of these. In addition, the combination of reverse genetics with next generation sequencing is applied to identify downstream genetic components of these signaling pathways and to discriminate between anterior and posterior targets in the early germ band.

With this work I propose a new model for the patterning of the antennal segment in Tribolium based on the cross regulation of head-gap genes, gap-genes and segment polarity genes. The RNAseq approach successfully identified target genes of the Wnt and hedgehog pathways, as confirmed via in situ hybridization, showing the great potential of this method. Finally, this study reveals an unexpected essential role of Tc-senseless in hindgut establishment.
\end{abstract}


Meiner Großmutter

Anna Bodner 


\section{Acknowledgements}

First of all I'd like to thank my supervisor Dr. Gregor Bucher. Thank you for the opportunity to work in this environment. Your open door policy was much appreciated, and helped a lot to keep my work focused and on track. Thank you for your faith in me when we started all this new and risky projects. Finally, thank you for your kind words, when they were needed the most.

I want to thank the members of my thesis committee, Dr. Andreas Wodarz and Dr. Steve Johnsen. You were a great help in stearing the project in the right direction.

A big thank you goes to Claudia Hinners, who put in a lot of effort in the daily lab work and thank you for bringing order into the chaos or for your never ending want of trying.

I'd like to thank Dr. Ernst Wimmer for asking the right questions during progress reports and personal meetings. You always made me rethink my point of view, which was a great benefit for my project and myself.

Thanks to Dr. Gabriela Salinas-Riester and the members of the TAL Göttingen for the NGS work.

I want to thank Dr. Tim Beissbarth for introducing me to the world of $\mathrm{R}$ and helping with statistical analysis of my data.

Special thanks to Stefan Dippel, Dr. Nico Posnien, Dr. Bernhard Schmid and Jonas Schwirz for the endless discussions and the tons of input resulting from 
them.

Thanks to Dr. Daniela Grossmann for helping out with injections and chocolate. Also, thanks to Kathrin Kanbach for prepping all those embryos and Elke Küster for providing me with fresh beetles.

Thanks to all my bachelor and master students (and Hiwi's), Nina Heckmann, Verena Nick, Christoph Schomburg, Anna Stief and Tobias Vollmer. You were a great help! Here is hoping you learned something, too.

I would like to thank all my lab members, Dr. Sebastian Kittelmann, Peter Kitzmann, Dr. Nikolaus Koniszewski, Janna Siemanowski, Julia Ulrich and Yong Gang. It was always fun to work in this atmosphere, even when it wasn't.

I am very grateful to the secretary staff, Birgit Rossi, Selen Pfändner and as of late Inga Schild and Bettina Hucke, for pulling me out when I was drowning in German bureaucracy. You are total angels!

Thanks to all the members of our department. It was great working with all of you for the last four years.

A big thank you goes to the open source software community. Without your endless effort in developing all these powerful software tools, this work would have never been accomplished.

Finally, I'd like to thank my mother Annemarie Oberhofer, who made all this possible. Thank you for your endless mental and financial support. You are the best! 


\section{Contents}

$\begin{array}{ll}\text { List of Figures } & \text { xvii }\end{array}$

List of Tables $\quad$ xix

1 Introduction $\quad 1$

1.1 Head Patterning . . . . . . . . . . . . . . . 2

1.1.1 Head patterning in Tribolium . . . . . . . . . . 3

1.1.2 Potential signaling centers in the Tribolium germ rudiment 5

1.2 Unraveling genetic networks with RNAseq . . . . . . . . . . 6

$\begin{array}{lll}2 & \text { Aims of the project } & 7\end{array}$

3 Materials \& Methods $\quad 9$

3.1 Strains . . . . . . . . . . . . . . . . . . . 9

3.2 Molecular Cloning . . . . . . . . . . . . . . . . . 9

3.3 Stainings . . . . . . . . . . . . . . . . . . . 10

3.3.1 Fixation ...................... 10

3.3.2 NBT-BCIP stainings with alkaline phosphatase . . . . . . 10

3.3.3 Double fluorescent in situ hybridization with tyramide signal amplification . . . . . . . . . . . . 11

3.3.4 NBT/BCIP-TSA double in situ stainings . . . . . . . . . 14

3.3.5 Immunohistochemistry . . . . . . . . . . . . . . . . . 14

3.3.6 Generation of a Tc-Armadillo1 antibody . . . . . . . . . 14

3.3 .7 Mounting ... . . . . . . . . . . . . 14

3.3 .8 Microscopy and Imaging . . . . . . . . . . . . . . . . . . 14

3.4 Heatshock mediated misexpression . . . . . . . . . . . . . 15 


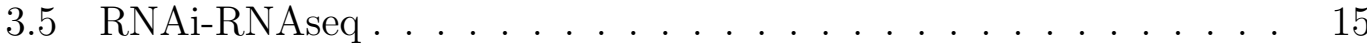

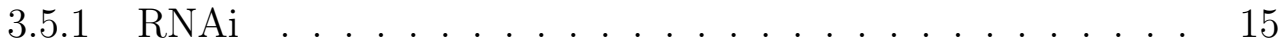

3.5 .2 RNA isolation . . . . . . . . . . . . . . . . . . . . . . . . . . .

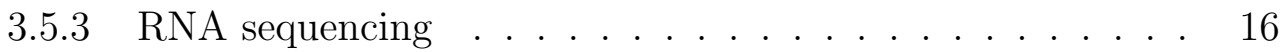

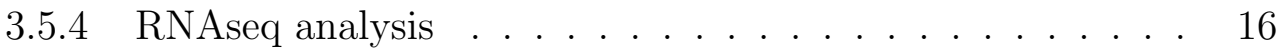

3.5.5 Annotation of RNAseq results . . . . . . . . . . . . 17

4 Results $\quad 19$

4.1 Establishment of parasegment boundaries in the head . . . . . . . 19

4.1.1 Hedgehog pathway effects . . . . . . . . . . . . . 20

4.1.2 Tc-knirps is needed for the establishment of the antennal parasegment boundary . . . . . . . . . . . 21

4.1.3 The role of Tc-empty spiracles in the stripe splitting process 24

4.2 Heatshock mediated misexpression of empty spiracles . . . . . . . 25

4.3 Differences in anterior and posterior interactions of the Wnt- and hedgehog Pathways . . . . . . . . . . . . . . 28

4.4 RNAi-RNAseq reveals differences in anterior and posterior target gene sets of the Wnt and hedgehog pathways . . . . . . . . . 30

4.4 .1 Quality control . . . . . . . . . . . . . . 30

4.4.2 In situ hybridization of candidate gene sets . . . . . . . . 34

4.4.3 Expression profiles of anterior expressed genes . . . . . . 41

4.5 RNAi screen of selected candidates . . . . . . . . . . . 43

4.6 A novel role for Tc-senseless in hindgut development in Tribolium 46

4.6.1 Tc-senseless is required for hindgut development in Tribolium 47

5 Discussion

5.1 Dynamic expression patterns in the establishment of the ocular and antennal parasegment boundaries . . . . . . . . . . 51

5.1.1 Genetic model for the patterning of the antennal segment . 52

5.1 .2 Outlook stripe splitting project . . . . . . . . . . 54

5.2 RNAseq after RNAi reveals differences in anterior and posterior target gene sets . . . . . . . . . . . . . . . . 56

5.2.1 Segment polarity interactions in head and growth zone . . 56

5.2.2 The ocular parasegment boundary as anterior signaling center 58 
5.2 .3 Posterior target genes . . . . . . . . . . . . . . . . . . . 59

5.2 .4 Outlook RNAseq . . . . . . . . . . . . . 60

$\begin{array}{ll}\text { References } & 61\end{array}$

$\begin{array}{ll}\text { A Clones and Primer } & 77\end{array}$

$\begin{array}{ll}\text { B RNAseq } & 81\end{array}$

B.1 Candidate Genes . . . . . . . . . . . . . . . . . 81

B.2 Gene ontology enrichment . . . . . . . . . . . . . 88

B.3 Tc-senseless phylogenetic tree . . . . . . . . . . . . 95

$\begin{array}{lr}\text { C Scripts } & 97\end{array}$

C.1 R . . . . . . . . . . . . . . . . . . . 97

C.1.1 DESeq Analysis . . . . . . . . . . . . . . . . . . . . . . . 97

C.1.2 Miscellaneous . . . . . . . . . . . . . . . . 100

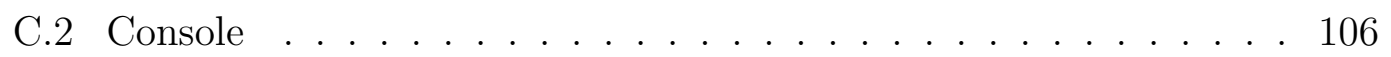

C.2.1 Mapping and counting RNAseq reads . . . . . . . . . 106

C.2.2 BLAST . . . . . . . . . . . . . . . 106

$\begin{array}{ll}\text { Curriculum Vitae } & 109\end{array}$

$\begin{array}{ll}\text { List of Publications } & 111\end{array}$ 


\section{List of Figures}

1.1 Segmentation in the insect head . . . . . . . . . . . . . . . 4

4.1 Segment polarity expression in the head . . . . . . . . . . 20

4.2 Double in situ stainings of Tc-hedgehog and Tc-wingless in $(A)$ Tc-cubitus interruptus ${ }^{R N A i}$ and $(B)$ Tc-smoothened ${ }^{R N A i} \ldots . . . .21$

4.3 Double in situ stainings of Tc-hedgehog and Tc-wingless in Tc-

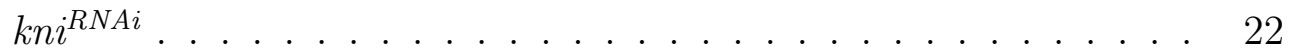

4.4 Double in situ stainings of Tc-knirps and Tc-wingless . . . . . . 23

4.5 Double fluorescent in situ stainings . . . . . . . . . . . . . . 24

4.6 Double in situ stainings of Tc-wingless and Tc-hedgehog in Tcempty spiracles ${ }^{R N A i} \ldots \ldots \ldots$. . . . . . . . . . . . . 25

4.7 Hatch rates of heatshocked transgenic (\#111) and wild type (VW) embryos ........................... 26

4.8 Phenotypes of empty spiracles overexpression . . . . . . . . . . 27

4.9 Complementary interactions of Wnt- and hedgehog signaling in the head and growth zone . . . . . . . . . . . . . . . . . . . . . 29

4.10 Subtractive RNAseq after RNAi . . . . . . . . . . . . . . . . 31

4.11 Quality controls of RNAseq experiment . . . . . . . . . . . . 33

4.12 MA-plots of RNAseq treatments . . . . . . . . . . . . . . . . 33

4.13 Wnt candidates in situ stainings . . . . . . . . . . . . . . . . . 38

4.14 Posterior hedgehog candidates . . . . . . . . . . . . . . . . 40

4.15 Fold change values of potential anterior target genes . . . . . . . . 41

4.16 Fold change values of target genes with anterior and posterior expression domains . . . . . . . . . . . . . . . . . . . . . . 43

4.17 Phenotype classes of RNAi screen . . . . . . . . . . . . . 45 
4.18 au2.g7984 dsRNA cuticle phenotype . . . . . . . . . . . . . 45

4.19 Expression of Tc-senseless . . . . . . . . . . . . . . . . 46

4.20 Tc-senseless ${ }^{R N A i} \ldots \ldots \ldots \ldots$. . . . . . . . . . . . . 48

5.1 Genetic model to establish the antennal parasegment boundary in Tribolium ......................... 55

5.2 Segment polarity interactions in head and growth zone . . . . . 57

B.1 Tc-senseless phylogenetic tree . . . . . . . . . . . 95 


\section{List of Tables}

3.1 TSA conjugate synthesis . . . . . . . . . . . . . . 11

A.1 Primer to clone genes and for dsRNA templates . . . . . . . . . . 77

A.2 Primer for clones obtained from colleagues . . . . . . . . . . . 78

A.3 Primer for the RNAseq in situ screen candidates . . . . . . . . . 78

A.4 Primer for non overlapping fragments of RNAseq in situ screen candidates . . . . . . . . . . . . . . . . . . 80

B.1 Posterior Wnt targets . . . . . . . . . . . . . . 81

B.2 Anterior Wnt targets . . . . . . . . . . . . . . 84

B.3 Posterior hedgehog targets . . . . . . . . . . . . . . 84

B.4 Anterior hedgehog targets . . . . . . . . . . . . . 88

B.5 Biological Process . . . . . . . . . . . . . . . . . . 89

B.6 Cellular Component _ . . . . . . . . . . . . . . . . . . . . . . 92

B.7 Molecular Function . . . . . . . . . . . . . . . . . . . . . . . . 93 


\section{1}

\section{Introduction}

Embryonic segmentation is a process well studied in the fruit fly Drosophila melanogaster. It starts at the syncytial blastoderm stage where the nuclei are not yet separated by cell walls. The fly employs the long germ mode of development [Liu and Kaufman, 2005] wherein all segments are patterned at this stage by the well known genetic cascade involving maternal, gap and pair-rule genes. Therefore, diffusion of transcription factors between nuclei is important during Drosophila patterning [Johnston and Nüsslein-Volhard, 1992]. After cellularization, signaling pathways are required to transmit external signals into the cells. During segmentation, the Wnt and hedgehog signaling pathways contribute to the setting up and the maintenance of the parasegment boundaries [NüssleinVolhard and Wieschaus, 1980]. Eventually, the Hox- genes give the segments their identity [McGinnis and Krumlauf, 1992].

Most insects, however, perform pattern formation mostly in a fully cellularized environment. For instance, abdominal segments are formed sequentially from a posterior elongation and differentiation zone (growth zone) [Tautz et al., 1994]. The red flour beetle Tribolium castaneum has become a major model for this short germ mode of embryogenesis and many differences to Drosophila have been found. The anterior morphogen bicoid is lacking [Stauber et al., 1999, Brown et al., 2001]. Instead, repression of canonical Wnt signaling at the anterior is essential for axis formation [Fu et al., 2012] and Tc-caudal translational repression is performed by Tc-mex3 and Tc-zen [Schoppmeier et al., 2009].

In the trunk, the gap gene orthologs do not directly position the pair rule stripes 


\section{INTRODUCTION}

[Bucher and Klingler, 2004, Cerny et al., 2005]. Instead, a pair rule gene circuit is at the core of an oscillating segmentation clock [Choe et al., 2006, El-Sherif et al., 2012, Sarrazin et al., 2012]. The terminal gap genes Tc-huckebein and the torso pathway do not appear to play a role in Tribolium head development [Schoppmeier and Schroeder, 2005, Kittelmann et al., 2013] while transcription factors from vertebrate neural plate patterning are involved [Posnien et al., 2011]. Several signaling pathways contribute to Tribolium growth zone patterning: Torso signaling is required for the establishment of the growth zone [Schoppmeier and Schroeder, 2005] and Wnt signaling components are needed for patterning of the growth zone and the segments [Bolognesi et al., 2008, Beermann et al., 2011] while FGF signaling is required for mesoderm formation during posterior elongation [Sharma et al., 2013]. Tc-hedgehog is expressed in the growth zone throughout segmentation without being required for elongation or segment specification but for segment maintenance [Farzana and Brown, 2008].

\subsection{Head Patterning}

The head is subdivided in a procephalic and gnathocephalic region (figure 1.1). The gnathocephalic part is patterned by the classic genetic cascade as explained above [Pankratz and Jäckle, 1990]. Patterning of the anterior head region must be different for some obvious reasons. Pair rule genes are not expressed there [Bucher and Wimmer, 2005] and the anterior most Hox gene (labial) is expressed in the intercalary segment [Abzhanov and Kaufman, 1999, Posnien and Bucher, 2010].

In Drosophila the terminal system (torso) and the bicoid morphogen gradient are the key factors to pattern anterior structures. It activates the so called head-gap genes orthodenticle, empty spiracles, buttonhead and sloppy paired which are involved in the patterning of the anterior head [Finkelstein and Perrimon, 1990, Cohen and Jürgens, 1990, Grossniklaus et al., 1992]. They are expressed in overlapping domains and loss of function mutations lead to the deletion of adjacent segments. The idea was that these genes define segment borders as well as their identity [Cohen and Jürgens, 1991] but this hypothesis could not be verified by 
ectopic expression of those genes [Gallitano-Mendel and Finkelstein, 1998, Wimmer et al., 1997].

Instead it was found that a second-order regulator, collier, acts between the head gap genes and the segment polarity genes, as do the pair-rule genes in the trunk [Crozatier et al., 1996, 1999]. For the intercalary segment it was shown that collier directly activates hedgehog by binding to a specific cis-regulatory element in the promoter region of hedgehog [Ntini and Wimmer, 2011a,b].

In summary the head gap genes, along with the terminal gap genes tailless and huckebein, regulate the expression of the segment polarity genes in the head [Mohler, 1995], although the underlying genetic network remains unclear. Further, the interactions of the segment polarity genes in the head are not the same as in the trunk region [Gallitano-Mendel and Finkelstein, 1997].

In spiders a wave of hedgehog expression is required for the metamerization of the anterior head [Pechmann et al., 2009]. Hedgehog is co-expressed with orthodenticle in a generative zone from which additional segments emerge via the traveling and splitting of the hedgehog expression domain [Kanayama et al., 2011]. However, the proposed auto-regulatory feedback loop of these genes does not include the input of other head-gap genes.

\subsubsection{Head patterning in Tribolium}

In Tribolium the functions of the head gap genes differ. In Tc-orthodenticle ${ }^{R N A i}$ the complete head is lost [Schröder, 2003], due to an early function in dorsoventral patterning [Kotkamp et al., 2010]. A later function of Tc-orthodenticle specifically affects parts of the procephalic head, whereas Tc-empty spiracles ${ }^{R N A i}$ leads to misoriented antennae and loss of eyes. Tc-buttonhead ${ }^{R N A i}$ has no effect on head formation [Schinko et al., 2008].

The classic gap gene knirps is not involved in head patterning in Drosophila whereas in Tribolium, it is needed for the establishment of the antennal and mandibular segments [Cerny et al., 2008]. Recently it was shown that Tc-knirps receives input from the pair rule gene even skipped being another difference to the Drosophila model wherein the gap genes act upstream of the pair-rule genes [Peel et al., 2013]. Tc-sloppy paired is expressed in narrow stripes compared to the 


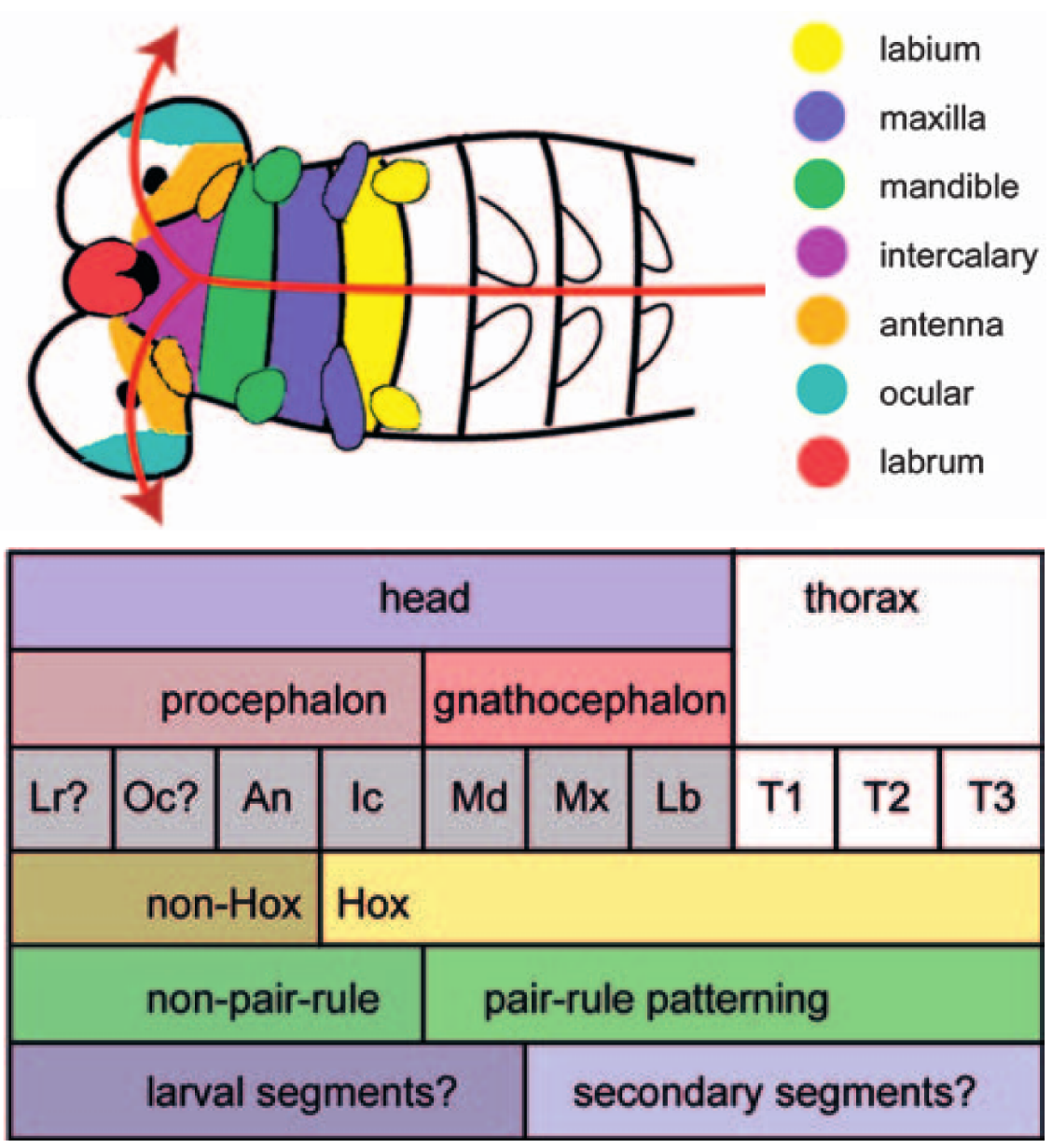

Figure 1.1: Segmentation in the insect head - Anterior head (procephalic): labrum, ocular, antenna, intercalary. Posterior head (gnathocephalic): mandible, maxilla, labium. No pair-rule genes and hox genes (from intercalary onwards) are expressed in the anterior head. Figure taken from [Bucher and Wimmer, 2005] 
gap gene-like expression in Drosophila and Tc-sloppy paired ${ }^{R N A i}$ shows additional defects in the abdomen [Choe and Brown, 2007].

With the lack of pair rule input the classic trunk segmentation model is not working [Posnien et al., 2010] and it remains unclear how parasegment boundaries are established in the head.

\subsubsection{Potential signaling centers in the Tribolium germ rudiment}

At the germ rudiment stage the signaling ligands Tc-wingless and Tc-hedgehog are expressed in adjacent domains in the head. This is the first postblastodermal stage and directly precedes posterior elongation and antennal segment formation suggesting that crucial signaling events are taking place.

The expression domains of orthologuos head patterning genes in the anterior region show a high similarity between insects and vertebrates [Wurst and BallyCuif, 2001, Urbach, 2007]. More recently the list of conserved head patterning genes and their similar expression domains compared to vertebrates was expanded in Tribolium and it was shown that several of these head specific genes are expressed in stripes parallel or overlapping the ocular parasegment boundary [Posnien et al., 2011]. Therefore, it is assumed that the ocular parasemgent boundary corresponds to the vertebrate mid-hind-brain boundary.

The other expression domain of Tc-wingless and Tc-hedgehog is in the growth zone. RNAi against crucial components of the Wnt pathway interferes with posterior elongation [Bolognesi et al., 2008, 2009] whereas disruption of the hedgehog pathway does not have an effect on the elongation process. However, later during development parasegment boundaries are not stable without a functioning hedgehog pathway and the germ band collapses during retraction [Farzana and Brown, 2008].

Taken together, the head and growth zone expression domains of these pathways are likely to be a part of anterior and posterior signaling centers. 


\subsection{Unraveling genetic networks with RNAseq}

The interactions of the Wnt and hedgehog pathway seem to be conserved in trunk segmentation between Drosophila and Tribolium [Oppenheimer et al., 1999]. However, the interactions of the pathways in the anterior head region and in the growth zone had not been well studied so far. Their respective target gene sets were not known and specifically, it had remained elusive in how far the target gene sets of these two pathways differ within and between these putative head and growth zone signaling centers.

In RNAseq a sample RNA is reverse transcribed and the resulting cDNA is used in massively parallel sequencing to yield millions of short reads in one single run. After mapping these reads back to a reference genome (transcriptome) the expression levels of all the transcribed genes in the sample are obtained [Wilhelm et al., 2008, Nagalakshmi et al., 2008, Mortazavi et al., 2008, Sultan et al., 2008].

In this work, I used this method to identify the target genes of the Wnt and hedgehog pathways, by comparing RNAi samples, where the pathways had been disrupted, to wild type embryos. The chosen developmental stage was the germ rudiment stage, where the trunk parasegment boundaries are not yet developed. This allows to examine the interactions and functions of the segment polarity signaling pathways independently from their function in the trunk. 


\section{2}

\section{Aims of the project}

The main aim of this study is to analyze the underlying genetic network of the early expression of the segment polarity genes Tc-wingless and Tc-hedgehog at the ocular parasegment boundary and in the growth zone. This includes

- The identification of upstream regulators

- A description of the interactions of the two pathways

- The identification of downstream target gene sets in order to compare them between head and growth zone

The first part is achieved by a candidate gene approach. Candidates are selected according to their expression pattern and time point and knocked down via RNAi. To study the interactions of the pathways their function is disrupted with RNAi. In both approaches the effect on the expression pattern of Tc-wingless and Tchedgehog is examined.

By a combination of RNAi against key components of the two pathways with RNAseq, downstream target genes can be identified genome wide. The resulting candidates are further examined in an in situ screen to verify their correct expression pattern. Finally, genes showing the predicted expression pattern are analyzed in more detail. 
2. AIMS OF THE PROJECT 


\section{3}

\section{Materials \& Methods}

\subsection{Strains}

Tribolium San Bernardino wild type strain was used for all experiments if not stated differently. Beetles were maintained at standard conditions $\left(32^{\circ} \mathrm{C}\right.$ on full grain flour supplemented with 5\% dry yeast) [Sokoloff, 1974]. Transgenic beetle line \# 111 used in heatshock experiments ${ }^{1}$ was generated with piggyback transgenesis in vermillion white and contained the Tc-empty spiracles open reading frame under the control of an endogenous heatshock promoter.

Black males were used for RNAi experiments with candidate genes from the RNAseq experiment. For egg collections beetles were maintained at $32^{\circ} \mathrm{C}$ on white flour supplemented with $5 \%$ dry yeast.

\subsection{Molecular Cloning}

All genes used in this thesis were cloned using standard techniques. Genes were amplified from $\mathrm{cDNA}^{2}$ with Phusion ${ }^{\mathrm{TM}}$ or advantageTaq ${ }^{\mathrm{TM}}$ and cloned into pJET1.2 or pCRII vector, respectively. Primers were designed using Primer3 software [Untergasser et al., 2012]. A complete list of all primers used in this thesis can

\footnotetext{
${ }^{1}$ Transgenic beetle line was generated by Johannes Schinko as detailed in [Schinko et al., $2012]$

${ }^{2}$ cDNA synthesis by Sebastian Kittelmann and Jonas Schwirz with the SMART PCR cDNA kit (ClonTech)
} 


\section{MATERIALS \& METHODS}

be found in the appendix (section A). The obtained sequences were verified using blastall [Altschul et al., 1997] in bioperl [Stajich et al., 2002] with the Tc-au2 gene set as the reference ${ }^{1}$.

\subsection{Stainings}

In Situ probes were synthesized with the DIG (Digoxigenin), FLU (Fluorescein) and BIO (Biotin) labeling kits from Roche using T7 or SP6 polymerases according to the manufacturers instructions.

\subsubsection{Fixation}

Embryos were dechorionated 2 times for 3 minutes in $50 \%$ commercial bleach in small sieves (mesh size $180 \mu \mathrm{m})$. Fixation was performed as described previously [Schinko et al., 2009]. The recipe for the fixation buffer was modified after [Sandmann et al., 2006]: 1 mM EDTA, 0.5 mM EGTA, $100 \mathrm{mM} \mathrm{NaCl,} \mathrm{2.5 \%}$ formaldehyde, $50 \mathrm{mM}$ HEPES, $\mathrm{pH} 8$.

\subsubsection{NBT-BCIP stainings with alkaline phosphatase}

In situ hybridization was performed as described previously [Schinko et al., 2009] with minor changes: $2 \%$ Roche blocking reagent was added to the HybeA buffer. PBT was substituted with maleic acid buffer (MABT) as the standard washing buffer $^{2}$. $2 \%$ Roche blocking reagent in MABT was used as the standard blocking buffer in all following steps.

\footnotetext{
${ }^{1}$ Gene set based on Tcas3.0 [Tribolium Genome Sequencing Consortium et al., 2008], reannotated by Mario Stanke with augustus software [Stanke and Waack, 2003], unpublished; hosted on bioinf.uni-greifswald.de

${ }^{2}$ see page 12 for the recipe
} 


\subsubsection{Double fluorescent in situ hybridization with tyra- mide signal amplification}

This protocol is an adaption of the [Lauter et al., 2011] zebrafish in situ protocol to the NBT-BCIP protocol described above. Detection is based on tyramide signal amplification (TSA).

\section{Synthesis of fluorescent tyramide conjugates}

$4 \AA$ molecular sieves (Fluka) are activated twice for two minutes in a microwave. $3 \mathrm{ml}$ DMF (dimethylformamide) are dried twice over the activated molecular sieves. $10 \mathrm{mg}$ tyramine $\mathrm{HCl}$ are dissolved in $990 \mu \mathrm{l}$ dry DMF containing $10 \mu \mathrm{l}$ triethylamine. Fluorescent dyes are modified to contain NHS-ester groups (Pierce, Dylight-NHS conjugates). $1 \mathrm{mg}$ NHS-conjugate is dissolved in $100 \mu \mathrm{l}$ dry DMF. The complete NHS solution and the tyramine solution are mixed at a 1.1:1 molar ratio (see table 3.1). The mixture is incubated in the dark for two hours and diluted to $1 \mathrm{ml}$ with ethanol afterwards.

Table 3.1: TSA conjugate synthesis

\begin{tabular}{|c|c|c|c|c|}
\hline Dye & $\operatorname{Mr}($ Dye $)[\mathrm{g} / \mathrm{mol}]$ & $\mathbf{n}(\mathbf{D y e})[\mu \mathrm{mol}]$ & $\mathbf{n}($ Tyramin $)[\mu \mathrm{mol}]$ & $\mathrm{V}($ Tyramin $)[\mu \mathrm{l}]$ \\
\hline Dylight 405 & 793 & 1.261 & 1.146 & 19.9 \\
\hline Dylight 488 & 1011 & 0.989 & 0.899 & 15.6 \\
\hline Dylight 550 & 1040 & 0.962 & 0.874 & 15.2 \\
\hline Dylight 633 & 1066 & 0.938 & 0.853 & 14.8 \\
\hline
\end{tabular}

\section{Buffers}

- HybeA, $100 \mathrm{ml}$ for 350 stainings:

$100 \mathrm{ml}$ HybeB, $0.1 \mathrm{ml}$ heparin [50 mg/ml], $0.5 \mathrm{ml}$ yeast RNA [20 mg/ml] and $2 \mathrm{ml}$ sonicated salmon sperm DNA $[20 \mathrm{mg} / \mathrm{ml}]$ are boiled for $10 \mathrm{~min}$ and cooled on ice for $3 \mathrm{~min}$.

Add $2 \mathrm{~g}$ dextran sulfate, $2 \mathrm{~g}$ Roche blocking reagent and $1 \mathrm{ml}$ Denhardts solution. Heat to $65^{\circ} \mathrm{C}$ until everything is dissolved (ca. $30 \mathrm{~min}$ ). Store at $-20^{\circ} \mathrm{C}$. 
- Denhardts solution: $20 \mathrm{mg}$ Ficoll, $20 \mathrm{mg}$ Polyvinylpyrrolidone and $20 \mathrm{mg}$ BSA in $1 \mathrm{ml} \mathrm{H}_{2} \mathrm{O}$

- 5 x MABT stock: $500 \mathrm{mM}$ maleic acid, pH to 7.5 with $\mathrm{NaOH}, 750 \mathrm{mM}$ $\mathrm{NaCl}, 0.5 \%$ Tween 20

- Blocking buffer : 1 x MABT, $2 \%$ Roche blocking reagent. Store at $4^{\circ} \mathrm{C}$.

- Borate buffer: $100 \mathrm{mM}$ boric acid, pH to 8.5 with $\mathrm{NaOH}$, $0.1 \%$ Tween 20

- TSA staining buffer, prepare freshly before use (100 $\mu \mathrm{l} /$ staining): borate buffer with $2 \%$ dextran sulfate, $450 \mu \mathrm{m} / \mathrm{ml}$ 4-iodophenol, $0.003 \% \mathrm{H}_{2} \mathrm{O}_{2}$ and $0.4 \mu \mathrm{l}$ tyramide conjugate. Protect from light if a fluorescent tyramide is used.

- Inactivation buffer: $100 \mathrm{mM}$ glycine, $\mathrm{pH}$ to 2 with $\mathrm{HCl}$, $0.1 \%$ Tween 20

Procedure The protocol starts at the pre-hybridization step. All previous steps are identical to [Schinko et al., 2009].

- Replace HybeB with $250 \mu \mathrm{l}$ HybeA and prehybridize for $1 \mathrm{~h}$ at $60^{\circ} \mathrm{C}$.

- Dilute your probes to the desired concentration in $30 \mu \mathrm{l}$ HybeA. Heat to $90^{\circ} \mathrm{C}$ for $2 \mathrm{~min}$ and place on ice for $5 \mathrm{~min}$. Preheat the probes to $60^{\circ} \mathrm{C}$.

- Remove HybeA, add the probes and incubate over night at $60^{\circ} \mathrm{C}$.

- Increase the temperature to $65^{\circ} \mathrm{C}$.

- Wash three times for 10 min with HybeB. Wash once with a 1:1 mixture of HybeB: MABT. Wash three times for 10 min with MABT.

- Switch to room temperature and wash three times for 10 min with MABT.

- Block for $1 \mathrm{~h}$ in blocking buffer. 
- Prepare a 1:2000 dilution ${ }^{1}$ of your HRP/POD conjugated antibody of choice in $1 \mathrm{ml}$ blocking buffer.

- Incubate with the antibody for at least $1 \mathrm{~h}$.

- Wash four times for 10 min with MABT. Wash over night at $4^{\circ} \mathrm{C}$ in blocking buffer.

- Wash four times for 10 min with MABT. Wash two times for 5 min with borate buffer.

- Prepare the TSA staining buffer during the borate washes. Stain for 30 to 60 min without agitation.

- Wash three times with MABT.

Continue with the inactivation step for another round of detection or skip to the final washing steps.

- Incubate for 15 min with inactivation buffer.

- Wash three times for 10 min with MABT.

- Block for $1 \mathrm{~h}$ in blocking buffer.

- Repeat antibody addition, washes and staining reaction for your second target as described above.

Final washes: Wash three times for 10 min with MABT. Wash over night at $4^{\circ} \mathrm{C}$ with MABT. Replace MABT, add $0.015 \%$ sodium azide as a preservative and store at $4^{\circ} \mathrm{C}$.

\footnotetext{
${ }^{1}$ Tested for Roche anti-Dig-POD, Roche anti-Fluo-POD and Jackson streptavidin-HRP; supplier suggestions of 1:200 or more resulted in background
} 


\subsubsection{NBT/BCIP-TSA double in situ stainings}

The alkaline phosphatase driven NBT/BCIP reaction was combined with the horseradish peroxidase mediated TSA reaction. Therefore, both antibodies could be added at the same time in the in situ staining without the need of an inactivation step. This method reduced the time to carry out a double in situ staining to that of a single staining. In double in situ stainings using the same enzyme, the harsh inactivation step often reduces the signal of the second staining, which is another advantage of the here described approach.

\subsubsection{Immunohistochemistry}

Immunostainings were carried out with the same protocol as the in situ hybridizations. The hybridization steps were omitted.

\subsubsection{Generation of a Tc-Armadillo1 antibody}

A 15 amino acid peptide of the C-terminus ${ }^{1}$ of $T c$-Armadillo1 was conjugated to KLH and injected in two rabbits. Peptide synthesis and immunization of rabbits was carried out by Eurogentec.

The obtained rabbit serum was used at a 1:10000 dilution in immunostainings [Panfilio et al., 2013].

\subsubsection{Mounting}

Embryos from in situ hybridizations and immuno stainings were mounted in $100 \%$ glycerol. Cuticles of first instar larvae were mounted in a 1:1 mixture of lactic acid and Hoyer's medium [Anderson, 1954] and incubated for two days at $65^{\circ} \mathrm{C}$.

\subsubsection{Microscopy and Imaging}

Cuticles were imaged on a Zeiss LSM780 using a $550 \mathrm{~nm}$ LASER. The resulting stacks were loaded into Amira 5.32. 4 steps of Blind deconvolution and gaussian

\footnotetext{
${ }^{1}$ Sequence: PQDNNQVAAWYDTDL
} 
smoothing were applied. Intensity levels were set to range from 5-200.

TSA double fluorescent in situ stainings were also imaged on a Zeiss LSM780 using $488 \mathrm{~nm}$ and $550 \mathrm{~nm}$ lasers. The stacks were imported in Amira v5.3.2 and 3D models were reconstructed with the voltex module.

NBT/BCIP single and NBT/BCIP - TSA double stainings were imaged on a ZEISS Axioplan2 using ImagePro v6.2 software. All images were assembled in Photoshop CS2. The levels for fluorescent in situ stainings were adjusted to increase intensity. NBT/BCIP stainings were not modified. All figures were imported in Inkscape for labeling and formatting.

\subsection{Heatshock mediated misexpression}

The transgenic beetle line \#111 was used to study overexpression of Tc-empty spiracles. Heatshock experiments were carried out as described previously [Schinko et al., 2012] . 0-24 hour old embryos were heatshocked for ten minutes at $48^{\circ} \mathrm{C}$. In the case of multiple heatshocks they were applied every two hours.

\subsection{RNAi-RNAseq}

\subsubsection{RNAi}

DsRNA was synthesized as described previously [Fu et al., 2012]. Lithium precipitation was used for templates longer than 400bp and phenol/chloroform extraction followed by isopropanol precipitation for shorter ones. DsRNA was injected in female pupae (RNAseq) or adults (downstream candidates). With this parental RNAi technique the phenotype is passed on to the offspring[Bucher et al., 2002]. All genes used in the RNAi-RNAseq procedure were published previously with no off target effects reported [Bolognesi et al., 2009, Beermann et al., 2011, Farzana and Brown, 2008, Bolognesi et al., 2008, Kotkamp et al., 2010, Schoppmeier and Schroeder, 2005]. 


\subsubsection{RNA isolation}

RNA of 10-11h old RNAi and wildtype embryos was extracted using Trizol (Ambion) according to the manufacturers protocol, followed by DNAse digestion with turbo DNAse (Ambion) and phenol/chloroform (Ambion, $\mathrm{pH}=6.9$ ) extraction.

\subsubsection{RNA sequencing}

Library preparation for RNA-Seq was performed using the TruSeq RNA Sample Preparation Kit (Illumina, Cat.No:RS-122-2002) starting from $400 \mathrm{ng}$ of total RNA. Accurate quantitation of cDNA libraries was performed by using the QuantiFluor dsDNA System (Promega). The size range of final cDNA libraries was determined applying the DNA 1000 chip on the Bioanalyzer 2100 from Agilent (280 bp). cDNA libraries were amplified and sequenced by using the cBot and HiSeq2000 from Illumina (Single Read; 1x50 bp and 1x100bp). Sequence images were transformed with Illumina software BaseCaller to bcl files, which were demultiplexed to fastq files with CASAVA v1.8.2. Quality check was done via fastqc (v. 0.10.0, Babraham Bioinformatics). All treatments were sequenced in biological triplicates.

Library preparation, quality control, sequencing and demultiplexing was carried out by the Transkriptom Analyse Labor Göttingen.

\subsubsection{RNAseq analysis}

The obtained fastq formatted Illumina reads were mapped to the Tribolium au2 gene set using bowtie2 (version 2.1.0) [Langmead and Salzberg, 2012] with the these settings: -q -D 20 -R 3 -N 1 -L 20 -i S,1,0.50. Reads were counted with samtools [Li et al., 2009] and combined in a counts table. Statistical analysis of the data and differentially expressed gene calling was performed in $\mathrm{R}$ [Team, 2013] using the DESeq (version1.12.0) [Anders and Huber, 2010] package from bioconductor [Gentleman et al., 2004]. Genes were considered to be differentially expressed if $\log 2$ fold change was $>=|1|$ and an adjusted $\mathrm{p}$ value $<0.1$. Intersects and Venn diagrams were build with the Overlapper.R function from the Bioconductor manuals by Thomas Girke [Girke]. Heatmaps were plotted with gplots 
[Bolker et al., 2013] and RColorBrewer [Neuwirth, 2011], barplots with ggplot2 [Wickham, 2009].

\subsubsection{Annotation of RNAseq results}

The au2 gene set was BLASTed against the gene set from Flybase [Gelbart et al., 1997] using BLAST [Altschul et al., 1997] implemented in bioperl [Stajich et al., 2002] and a custom perl script ${ }^{1}$. Reciprocal best BLAST scores were reported as orthologs. Hits with an $E-$ value $<10^{-5}$ are considered homologs. The resulting list of au2 gene IDs with their corresponding Fbpp ID was merged to the Drosophila GO annotations downloaded from Flybase (GOC Validation Date: $01 / 25 / 2013)$ in $\mathrm{R}$.

\footnotetext{
${ }^{1}$ Perl script written by Mario Stanke and downloaded from bioinf.uni-greifswald.de. The script was modified to allow for multi threaded BLAST search.
} 


\section{4 \\ Results}

\subsection{Establishment of parasegment boundaries in the head}

The ocular parasegment boundary is the first to be established in the Tribolium embryo. To learn more about its initiation, the differences in segment polarity expression patterns were investigated in wild type and different RNAi knock down situations.

Double fluorescent in situ hybridizations (FISH) was used to get the expression topologies of Tc-wingless and Tc-hedgehog in the head. After the ocular parasegment boundary was established, the Tc-hedgehog domain became broader, extended backwards and splitted up. This process gave rise to the antennal Tchedgehog domain. Later the antennal Tc-wingless expression arised de novo adjacent to Tc-hedgehog (figure 4.1).

I tried to understand the underlying genetic mechanisms by looking at candidate genes that modify the stripe splitting. In this section the term head was used in a simplified way, including the antennal and ocular parasegment boundaries only. The complete process was described with these terms:

1. Establishment at the ocular domain

2. Stripe broadening and backwards extension

3. Stripe splitting 


\section{RESULTS}
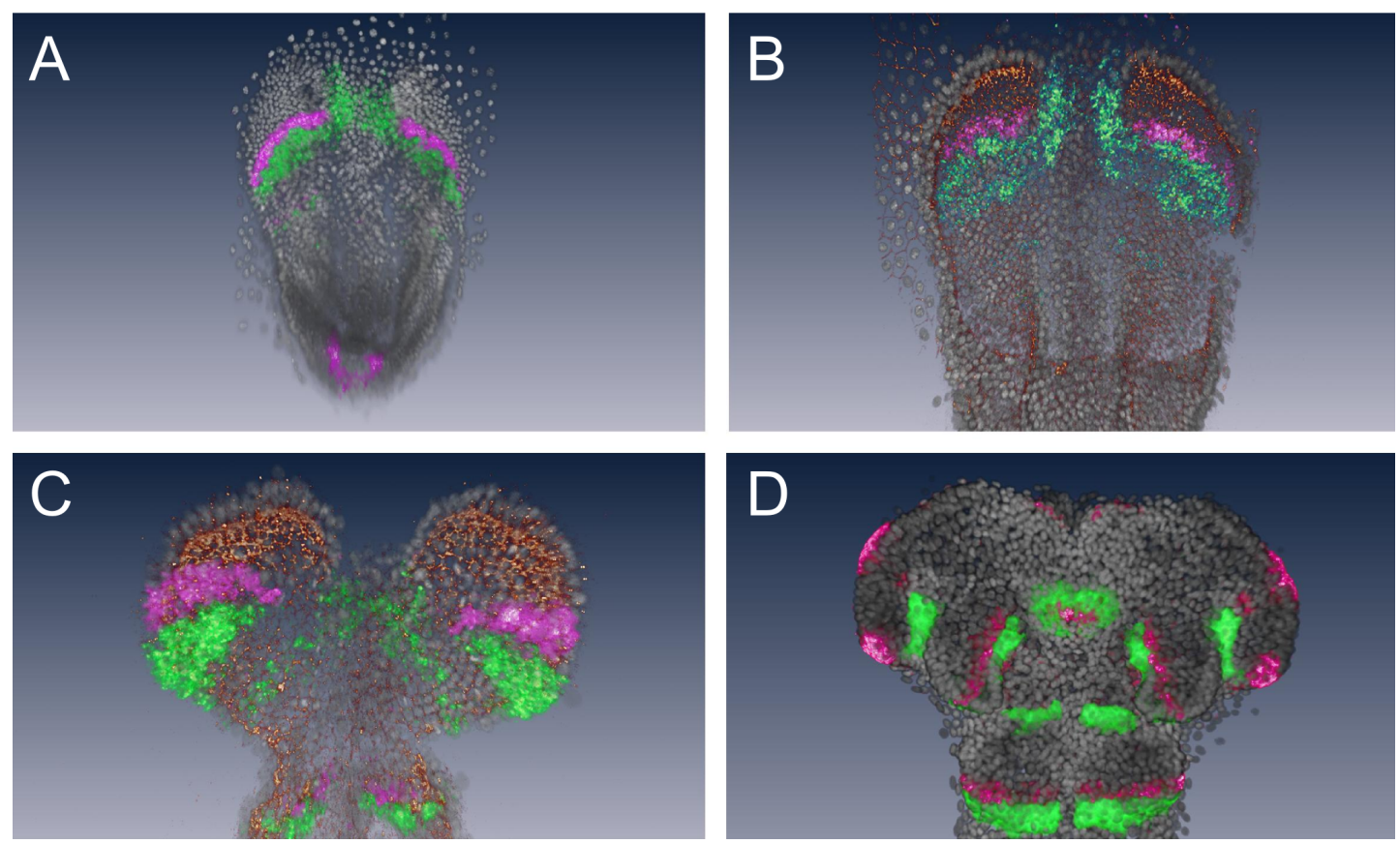

Figure 4.1: Segment polarity expression in the head - FISH stainings at different developmental timepoints ( $A$ to $C$ dorsal views, $D$ ventral view, anterior to the top) of Tc-wingless (magenta) and Tc-hedgehog (green) showing the dynamic expression pattern of Tc-hedgehog. Nuclei were colored in gray, Tc-Armadillo1 antibody staining in brown. (A) Initial expression of Tc-hedgehog and Tc-wingless at the ocular parasegment. (B) The ocular Tc-hedgehog domain becomes broader, extends backwards and starts to split up. $(C)$ Split process was completed and antennal Tc-hedgehog was established. (D) Antennal Tc-wingless arises de novo (ventral view)

\subsubsection{Hedgehog pathway effects}

To check for auto regulation of Tc-hedgehog expression in the head I disrupted the hedgehog pathway via Tc-cubitus interruptus ${ }^{R N A i}$ and Tc-smoothened ${ }^{R A A i}$. In the absence of hedgehog signaling cubitus interruptus was cleaved to its repressive form [Aza-Blanc et al., 1997]. In Tc-smoothened ${ }^{R N A i}$ hedgehog targets were always repressed, whereas Tc-cubitus interruptus ${ }^{R N A i}$ additionally derepressed hedgehog targets in cells without hedgehog signaling.

In both treatments the Tc-wingless expression domains were fading in the trunk 
indicating the important role of the hedgehog pathway in stabilizing parasegment boundaries. Ocular Tc-wingless seems unaffected while the expression in the growth zone was lost in Tc-cubitus interruptus ${ }^{R N A i}$. In Tc-smoothened ${ }^{R N A i}$ it was the other way round (figure 4.2). In addition in Tc-smoothened ${ }^{R N A i}$ the broad anterior Tc-hedgehog domain indicates that the stripe splitting failed (see also figure 4.9).
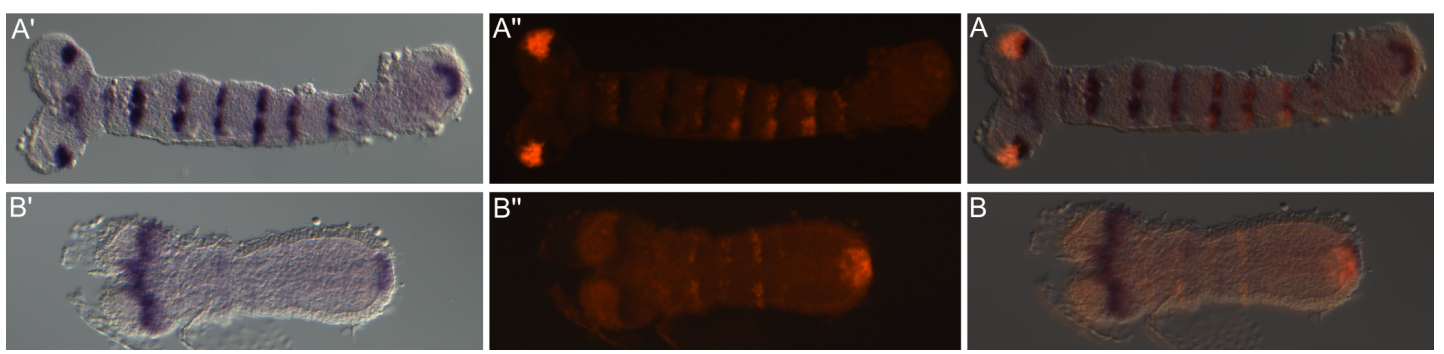

Figure 4.2: Double in situ stainings of Tc-hedgehog and Tc-wingless in (A) Tc-cubitus interruptus ${ }^{R N A i}$ and (B) Tc-smoothened ${ }^{R N A i}-$ Tc-hedgehog in blue (NBT/BCIP) andTc-wingless in red (TSA-Dylight550). (A) Tc-cubitus interruptus $^{R N A i}$; The antennal and ocular Tc-hedgehog domains were unaffected. Ocular Tc-wingless was unaffected, but antennal Tc-wingless was lost along with most trunk and the growth zone domains. (B) Tc-smoothened ${ }^{R N A i}$; The anterior Tc-hedgehog domain was broadened, trunk domains were lost but the growth zone was unaffected. Tc-wingless expression was lost in the head, reduced or fading in the trunk but unaffected in the growth zone.

In summary the hedgehog pathway was required for the stripe splitting process, but not for the establishment of Tc-hedgehog expression in the head. The trunk parasegment boundaries were fading without hedgehog signaling. Growth zone expression of the segment polarity genes was unaffected only in Tc-smoothened ${ }^{R N A i}$, but strikingly, Tc-wingless expression was lost in Tc-cubitus interruptus ${ }^{R N A i}$ without affecting elongation.

\subsubsection{Tc-knirps is needed for the establishment of the antennal parasegment boundary}

From previously published data it was clear that Tc-knirps plays a major role in establishing the antenna anlagen. Antennal expression of Tc-hedgehog and Tc- 


\section{RESULTS}

wingless was lost in Tc-knirps ${ }^{R N A i}$ (see Figure 4.3 and [Cerny et al., 2008, Peel et al., 2013]). This makes Tc-knirps a good candidate for the activation of Tchedgehog during the backwards extension of the expression domain. The ocular parasegment boundary was not affected by $T c-k n i r p s^{R N A i}$, whereas the dynamic Tc-hedgehog expression, leading to the fromation of the antennal parasegment boundary, was abolished.
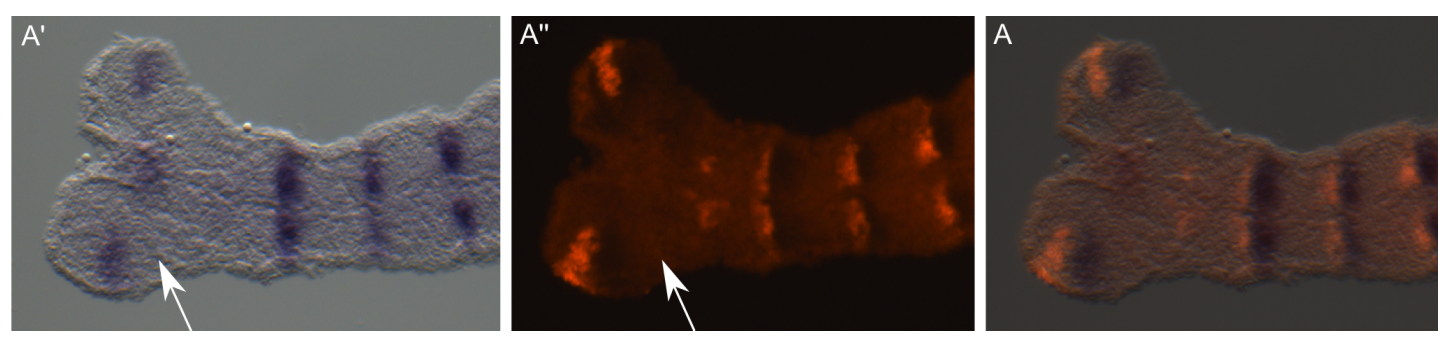

Figure 4.3: Double in situ stainings of Tc-hedgehog and Tc-wingless in $\boldsymbol{T} \boldsymbol{c}-\boldsymbol{k n \boldsymbol { n }} \boldsymbol{i}^{\boldsymbol{R} \boldsymbol{A} \boldsymbol{i}}-\left(A^{\prime}\right)$ Tc-hedgehog (NBT/BCIP) expression, antennal expression missing (arrow). ( $\left.A^{\prime \prime}\right)$ tc-wingless (TSA-Dylight550) expression, antennal expression missing (arrow). $(A)$ Overlay

For Tc-knirps to be the upstream activator of Tc-hedgehog during its movement from the ocular to the antennal domain, it should show similar expression dynamics. Double in situ stainings of Tc-wingless and Tc-knirps showed adjacent expression domains in very early germ bands before the serosa window had formed. As the serosa window began to close, the two expression domains became separated from each other. At later timepoints, as shown by the smaller serosa window, the gap between the domains increased further (see figure 4.4).

In summary, Tc-knirps function was essential for Tc-hedgehog expression at the antennal parasegment boundary and its early expression domain showed the same backwards extension. 

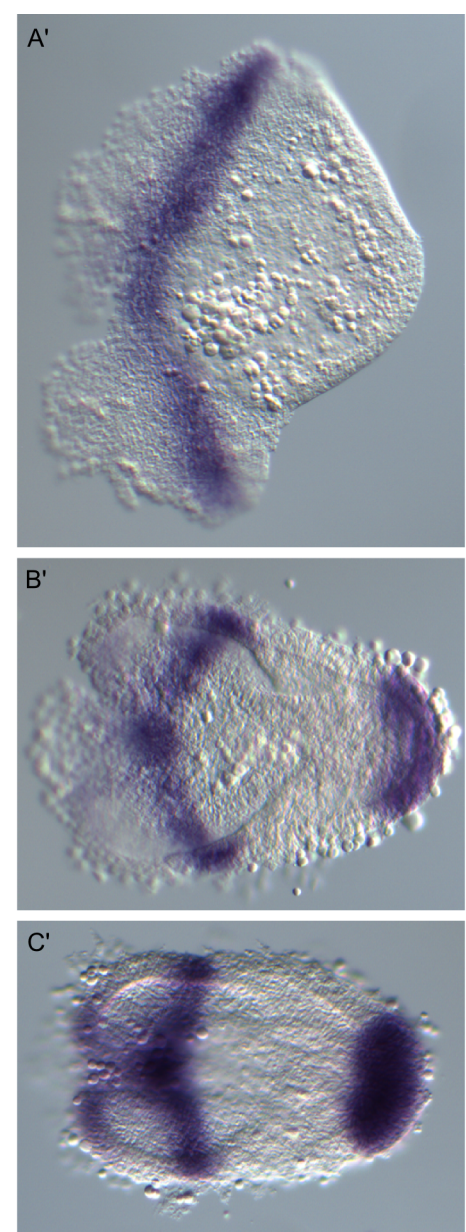

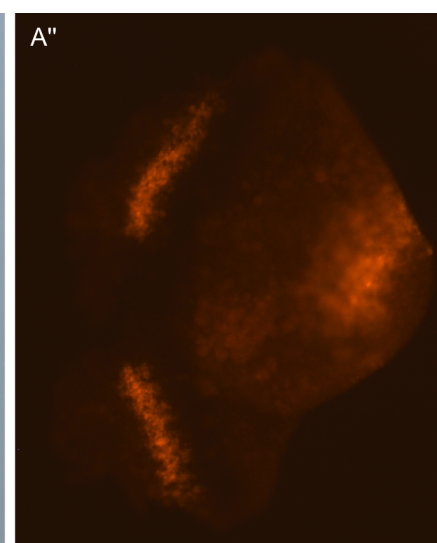

B"

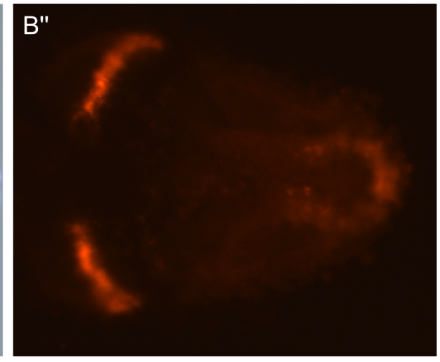

C"

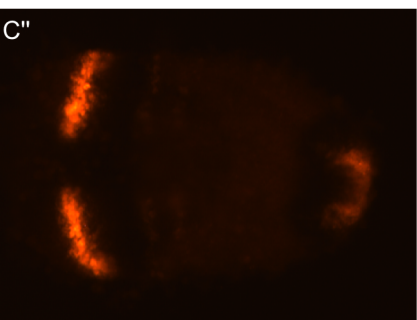

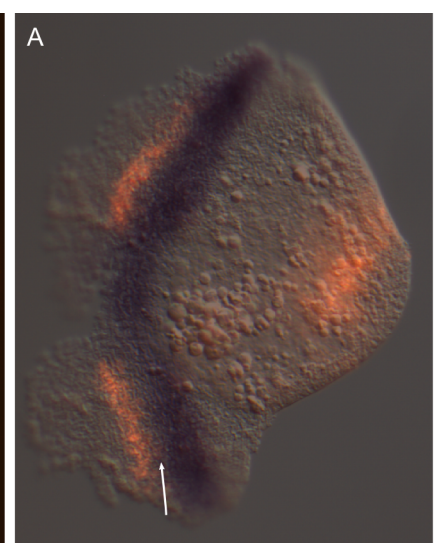
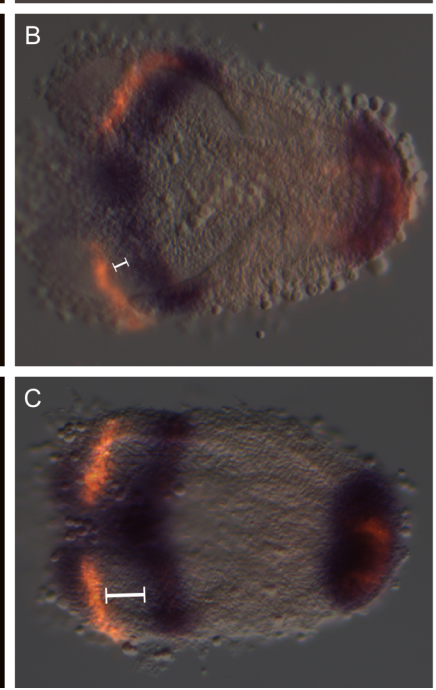

Figure 4.4: Double in situ stainings of Tc-knirps andTc-wingless - Tcknirps in blue (NBT/BCIP) $\left(A^{\prime}-C^{\prime}\right)$, Tc-wingless in red (TSA-Dylight550) $\left(A^{\prime \prime}-C^{\prime \prime}\right)$ in early germbands. Panels were sorted from youngest $(A)$ to oldest $(C)$ as marked by the size of the serosa window. ( $A$ ) No serosa window, adjacent expression domains (arrow in $A$ ). $(B)$ Slightly older germ band with formed serosa window. Gap between Tc-wingless and Tc-kni domains increased. $(C)$ Gap increased further 


\subsubsection{The role of Tc-empty spiracles in the stripe split- ting process}

In wild type embryos, Tc-empty spiracles was expressed exactly between the ocular and antennal Tc-wingless domains. In Tc-empty spiracles ${ }^{R N A i}$ the antenna of first instar larva were misoriented and the eyes were absent [Schinko et al., 2008]. The backwards extension and splitting of Tc-hedgehog occured in this field. After the broadening, the Tc-hegdehog domain has moved beyond the Tc-empty spiracles field, and the antennal Tc-wingless domain arised de novo (see figure 4.5).

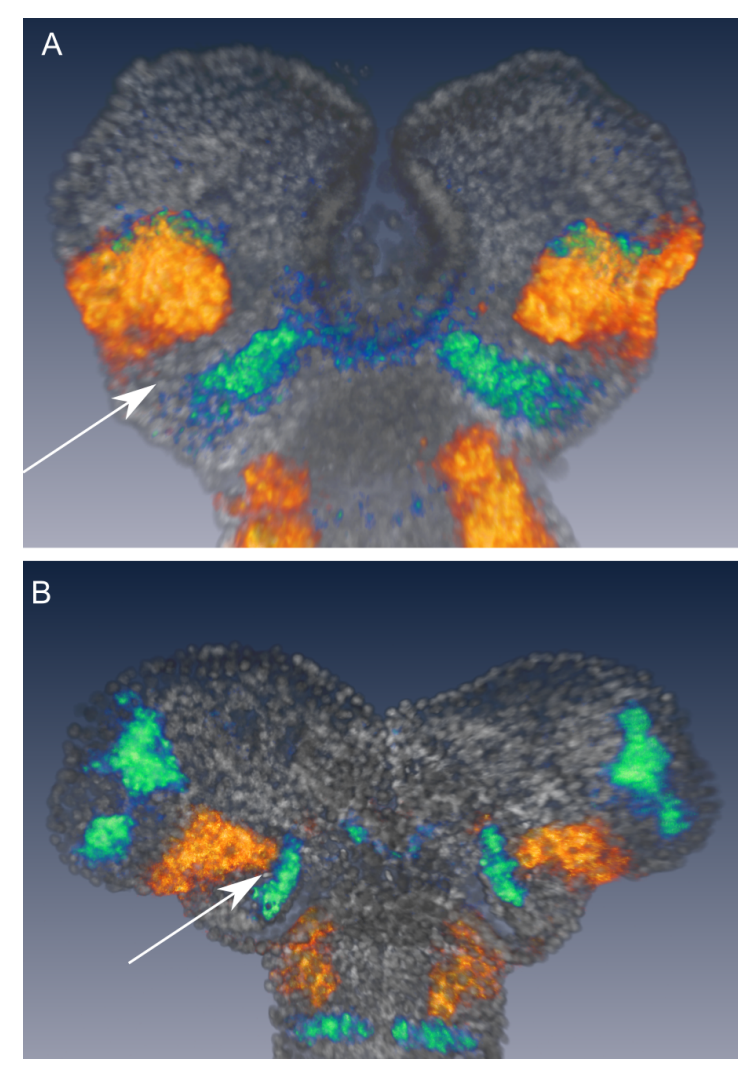

Figure 4.5: Double fluorescent in situ stainings - (A) Tc-empty spiracles in orange (TSA-Dylight550) and Tc-hedgehog in green (TSA-Dylight488). The arrow marks the gap between antennal Tc-hedgehog expression and the Tc-empty spiracles domain, where the new Tc-wingless domain arised. (B) Tc-empty spiracles in orange (TSA-Dylight550) and Tc-wingless in green (TSA-Dylight488). The arrow marks the adjacent expression domains of Tc-empty spiracles and Tc-wingless. 
In Tc-empty-spiracles ${ }^{R N A i}$, the initial ocular Tc-hedgehog expression (figure 4.6 A) and the backwards extension were unaffected. Due to the loss of the repressive effect of Tc-empty spiracles on Tc-wingless, all cells between the ocular and antennal segment became Tc-wingless positive and ocular Tc-hedgehog was lost. As a consequence the ocular parasegment boundary was lost which explains the phenotype described in [Schinko et al., 2008].
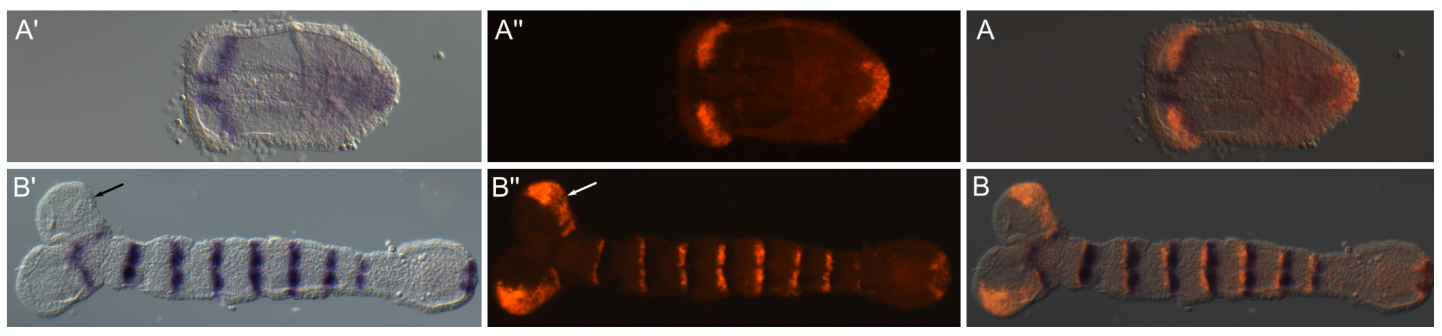

Figure 4.6: Double in situ stainings of Tc-wingless and Tc-hedgehog in Tc-empty spiracles ${ }^{\boldsymbol{R N A i}}$ - (Panel A) Early Tc-empty spiracles ${ }^{R N A i}$ embryos. $\left(A^{\prime}, A^{\prime \prime}\right)$ The ocular parasegment was established. Tc-wingless began to expand towards posterior (compare to the narrow wild type Tc-wingless domain in $4.1 \mathrm{~A}$ ). (Panel $B$ ) In elongating germ bands ocular Tc-hedgehog was missing (arrow in $B^{\prime}$ ) whereas $T c$-wingless expanded all the way from the ocular domain to the antennal Tc-hedgehog expression border (arrow in $\left.B^{\prime}\right)$. $(B)$ overlay

In conclusion, Tc-empty spiracles had no effect on the backwards moving of $T c$ hedgehog, but played an important role in hindering Tc-wingless to do the same, which led to the loss of the ocular Tc-hedgehog domain.

\subsection{Heatshock mediated misexpression of empty spiracles}

To confirm the repressive effect of Tc-empty spiracles on Tc-wingless, I analyzed a transgenic beetle line, where Tc-empty spiracles was driven ubiquitously via heatshock ${ }^{1}$.

In a pretest the experimental conditions were optimized. The transgenic line

\footnotetext{
${ }^{1}$ The transgenic line was generated by Johannes Schinko as described in [Schinko et al., 2012], but with the Tc-empty spiracles open reading frame instead of Tc-orthodenticle1
} 


\section{RESULTS}

(\#111) and the background strain (vermillion white, VW) were kept at $32^{\circ} \mathrm{C}$ and subjected to a different amount of heatshocks at $48^{\circ} \mathrm{C}$. Heatshocks were applied for ten minutes followed by a regeneration time of two hours at $32^{\circ} \mathrm{C}$. The maximum amount of heatshocks tested was four. Each experiment was repeated four times with new embryos. Figure 4.7 shows box plots of hatch rates for the transgenic line and the control (injection strain of the transgenic line). Hatch rates were decreasing with increasing number of heat shocks and approached zero for four heatshocks. However, in the the wild type strain the hatch rate started to decrease significantly with three or more heatshocks, too. Therefore, two heatshocks was considered as the best trade off between high effects of the heatshock in the transgenic line compared to low effects in the control.

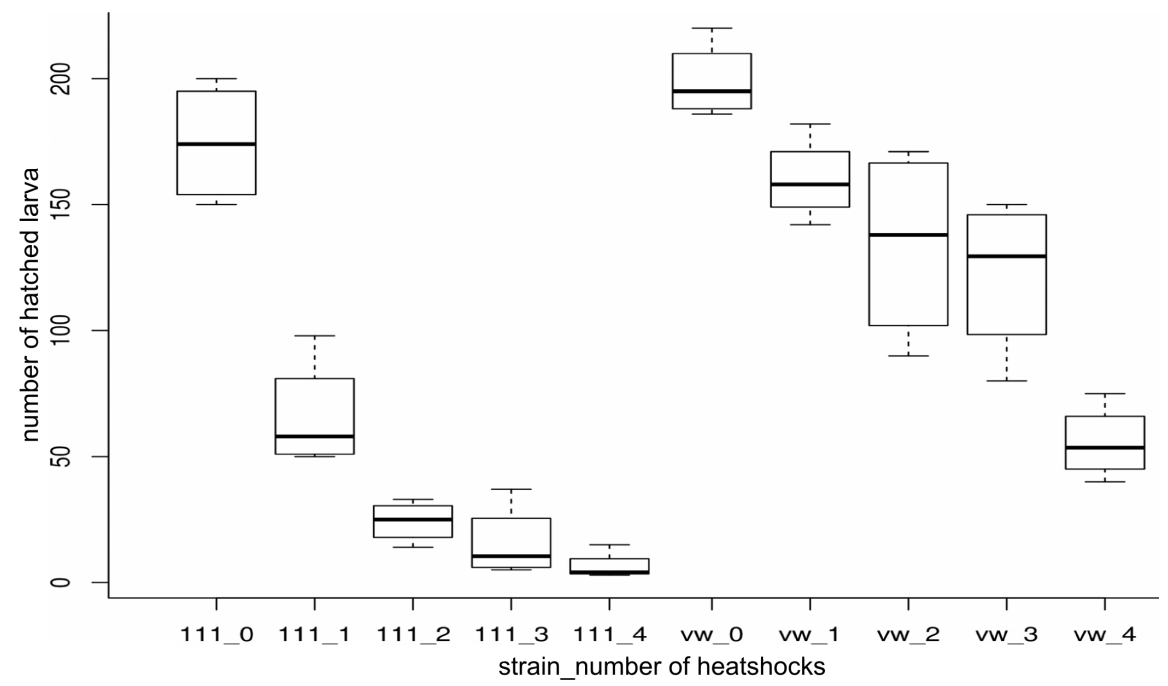

Figure 4.7: Hatch rates of heatshocked transgenic (\#111) and wild type (VW) embryos - Y-axis shows the absolute numbers of hatched larva. X-axis shows the type of embryo (\#111 or VW) followed by the amount of consecutive heat shocks $(0-4)$

Initial phenotype analysis showed some dramatic effects of Tc-empty spiracles overexpression. Segmentation was disrupted and bristles were misoriented in the whole larva. Interestingly, a small number developed ectopic antennae in the head (figure $4.8 A-C$ ). Double in situ stainings of Tc-hedgehog and Tc-wingless as segmental markers were performed. Tc-hedgehog was expressed in a single broad domain in the head lobes, but was depleted in the rest of the germ band. Ocular 
Tc-wingless was reduced and spotty. The trunk Tc-wingless expression domains were not able to build stable segmental boundaries and collapsed, whereas the expression domain in the growth zone seemed unaffected (figure $4.8 \mathrm{D}$ ).
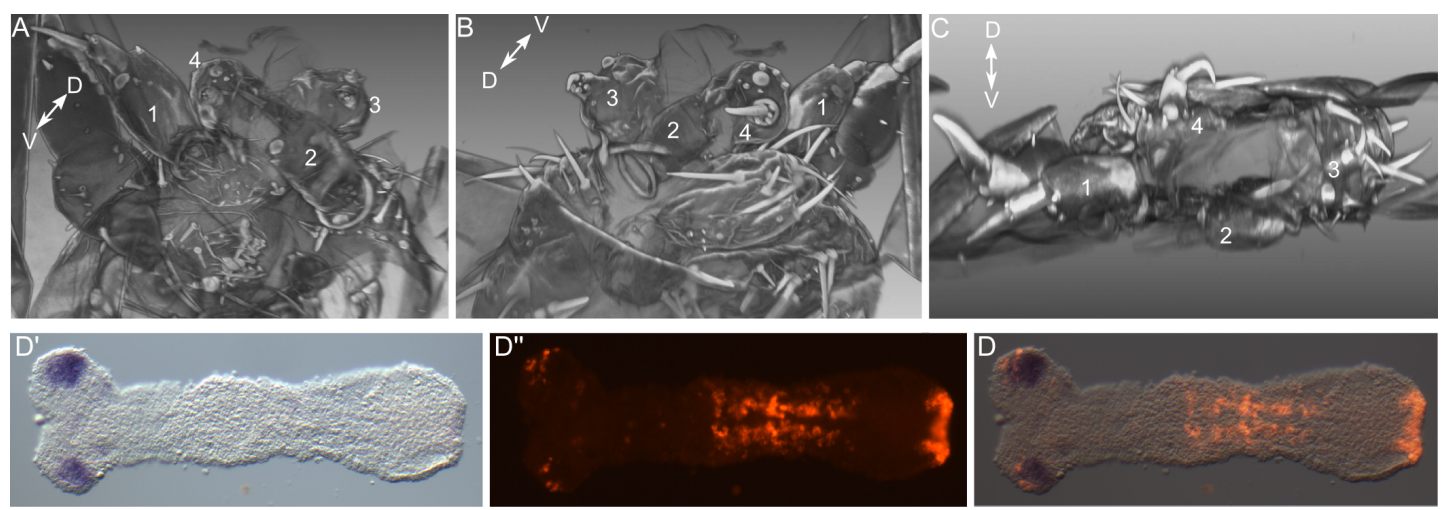

Figure 4.8: Phenotypes of empty spiracles overexpression - $(A-C)$ 3D reconstruction of cuticle phenotypes with $\mathrm{D} \leftrightarrow \mathrm{V}$ indicating the dorsal-ventral axis. $(D)$ In situ staining of elongating germ band. (A) Ventral view of a cuticle 3D model. Head shows 4 antennae, labeled 1-4. Antenna 1 and 2 look rather normal. Antenna 3 and 4 were smaller and located in the labrum. $(B)$ Dorsal view showing the antennae on the labrum $(3,4)$. $(C)$ View from the anterior $\left(D^{\prime}\right)$ Tc-hedgehog staining (NBT/BCIP). Only a single broad Tc-hedgehog domain remains in the head lobes. ( $\left.D^{\prime \prime}\right)$ Tc-wingless staining (TSA-Dylight550). Some remnants of ocular Tc-wingless expression remain, whereas trunk stripes have collapsed. The growth zone was not affected. (D) Overlay

Taken together, Tc-empty spiracles leads to the $\operatorname{loss}^{1}$ of anterior Tc-wingless domains in line with the expansion of Tc-wingless in the knock down situation. The ocular Tc-hedgehog domain expanded backwards, but failed to split up. Additionally, the overexpression led to the development of ectopic antennae. From the position of these antennae it seems that the labrum was transformed. However, this novel role of Tc-empty spiracles needs to be confirmed in further studies.

\footnotetext{
${ }^{1}$ reduction in the ocular domain
} 


\subsection{Differences in anterior and posterior inter- actions of the Wnt- and hedgehog Pathways}

In the trunk the mutual activation of the segment polarity genes wingless and hedgehog led to the stabilization of parsegment boundaries. However, these interactions were different in the anterior head region in Drosophila [Gallitano-Mendel and Finkelstein, 1997]. Therefore, I checked the cross-regulation of these genes in Tribolium and included the posterior growth zone in the analysis, an embryonic structure not present in Drosophila.

Tc-wingless and Tc-hedgehog regulation was examined in the head and growth zone regions of early (10-11 h old) and elongating (12-15 h old) germ bands in wild type and RNAi knock down embryos. RNAi embryos included:

- $T c$-arrow ${ }^{R N A i}$ disrupting the canonical Wnt pathway (figure $4.9 B_{1}-B_{4}$ ).

- Tc-hedgehog ${ }^{R N A i}$ disrupting the hedgehog pathway for Tc-wingless stainings (figure $4.9 C_{1}, C_{2}$ ).

- Tc-smoothened ${ }^{R N A i}$ disrupting the hedgehog pathway for Tc-hedgehog stainings (figure $4.9 C_{3}, C_{4}$ ).

Strikingly, the interactions were complementary: hedgehog signaling acts upstream of Tc-wingless expression in the head (figure $4.9 C_{1}, C_{2}$ ) but Wnt signaling controls Tc-hedgehog expression in the growth zone (figure $4.9 B_{3}, B_{4}$ ). Autoregulation was apparent only for the Wnt pathway in the growth zone, as indicated by the loss of Tc-wingless in Tc-arrow ${ }^{R N A i}$ but not in Tc-hedgehog ${ }^{R N A i}$ (figure $\left.4.9 B_{1}, B_{2}\right)$. Wnt signaling was also needed to maintain ocular but not antennal Tc-hedgehog (figure $4.9 \mathrm{~B}_{3}$ ). I do not find an indication for mutual activation like in the trunk parasegment boundaries of Drosophila. In Tc-smoothened ${ }^{R N A i}$ antennal and ocular Tc-hedgehog expression were fused (figure $4.9 C_{3}, C_{4}$ ). The trunk Tc-hedgehog domains were reduced and were fading completely in stronger RNAi's (compare trunk stripes in $A_{4}$ with $C_{4}$, also shown in figure $4.2 B^{\prime}$ ). 

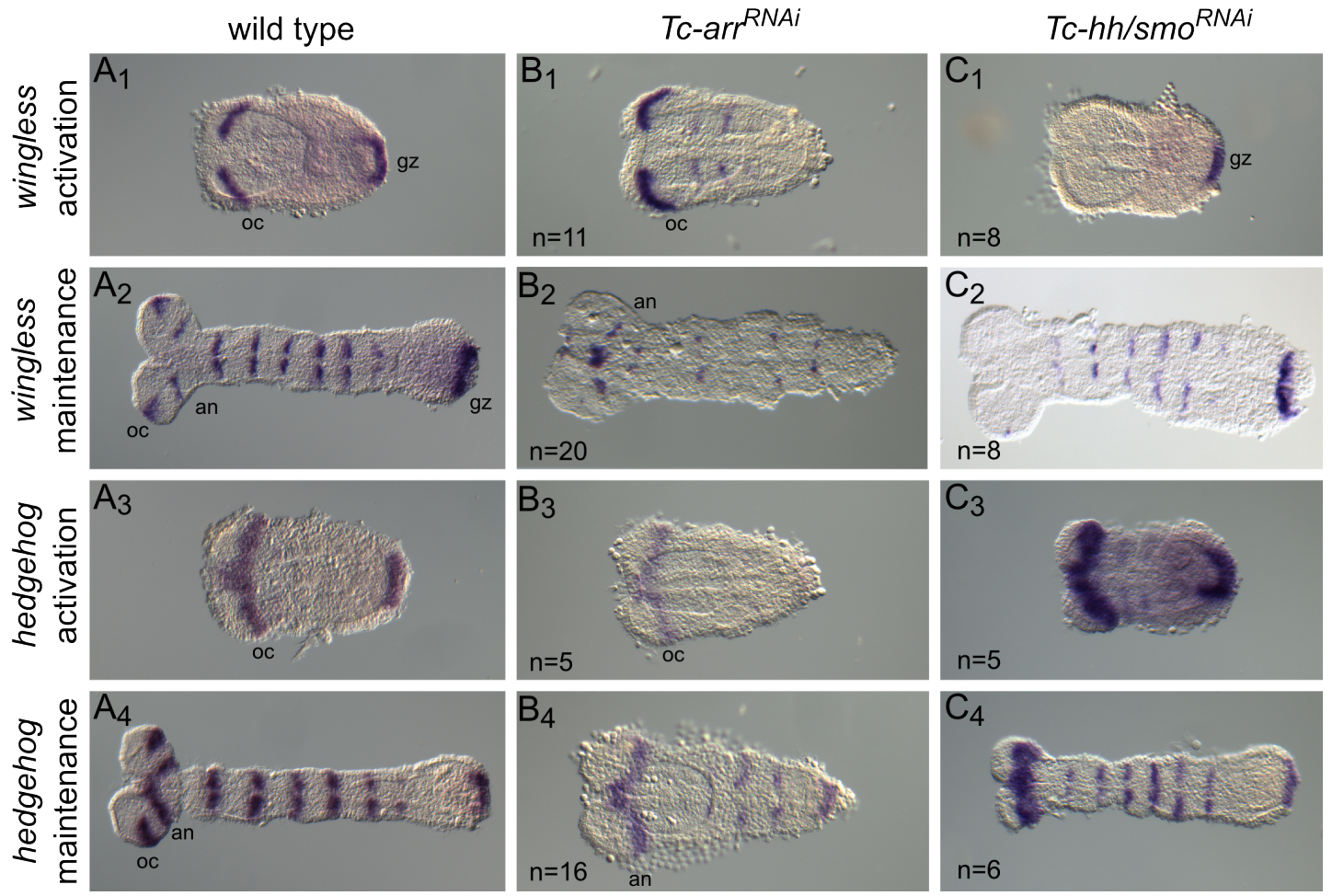

Figure 4.9: Complementary interactions of Wnt- and hedgehog signaling in the head and growth zone - Expression of Tc-wingless (rows 1 and 2) and Tc-hedgehogh (rows 3 and 4 ) in wildtype $\left(A_{1}-A_{4}\right)$ and RNAi treated embryos with interrupted Wnt $\left(B_{1}-B_{4}\right)$ or hedgehog pathway $\left(C_{1}-C_{4}\right)$ in germ rudiments (row 1 and 3 ) and elongating germ bands (row 2 and 4 ) with anterior oriented to the left. $\left(B_{1}-B_{4}\right)$ Wnt pathway disrupted: Both Tc-wingless and Tc-hedgehog expression were abolished in Tc-arrow ${ }^{R N A i}$ embryos in the growth zone. $\left(C_{1}-C_{4}\right)$ Hedgehog pathway disrupted: $\left(C_{1}-C_{2}\right)$ Tc-wingless expression was missing in the head of Tc-hedgehog ${ }^{R N A i}$ embryos. $(C 3-C 4)$ Ocular and antennal Tc-hedgehog expression domains were fused in $T c$-smoothened ${ }^{R N A i}$. Trunk expression domains were reduced. 


\subsection{RNAi-RNAseq reveals differences in ante- rior and posterior target gene sets of the Wnt and hedgehog pathways}

To identify target gene sets of the Wnt and hedgehog signaling pathways, I knocked down pathway components and identified the genes down-regulated in germ rudiments (10-11h, figure $4.9 A_{1}$ ) by comparing their transcript expression levels to wild type controls. The hedgehog pathway was disrupted by $T c$ hedgehog ${ }^{R N A i}$, canonical Wnt signaling was suppressed by $T c$-arrow ${ }^{R N A i}$. In order to reduce the resulting large candidate gene set for the Wnt pathway, I added $T c$ frizzled1/ $2^{R N A i}$ and $T c$-wntless ${ }^{R N A i}$ treatments. In addition I included treatments where either the head (Tc-orthodenticle $\left.{ }^{R N A i}\right)$ or the growth zone $\left(T_{c}\right.$-torso ${ }^{R N A i}$ ) Tc-wingless and Tc-hedgehog domains were depleted (figure $4.10 \mathrm{C}$-F) (shown for Tc-wingless in [Schinko et al., 2008, Schoppmeier and Schroeder, 2005]). This allowed identifying head versus growth zone specific target genes. For example, those $T c$-hedgehog target genes, which were in addition down-regulated in Tc-torso ${ }^{R N A i}$ but not in Tc-orthodenticle ${ }^{R N A i}$ were considered exclusive posterior targets of hedgehog signaling (figure 4.10).

\subsubsection{Quality control}

Three biological replicates were sequenced per treatment. A cluster analysis revealed that all treatments clustered together with the exception of Tc-hedgehog ${ }^{R N A i}$. The Tc-hedgehog libraries were not sequenced on the same day, which resulted in a clustering of sequencing days instead of treatments (figure $4.11 \mathrm{~A}$ ). Cuticle analysis of siblings of the sequnenced animals confirmed the high penetrance of the RNAi treatments (see figure $4.11 B$ for details). The RNAi knockdowns resulted in a transcript reduction of the targeted genes by 80-90\%, except for Tc-frizzled2 with $40 \%$ reduction (figure $4.11 C$ ).

In the RNAseq analysis a gene was considered downregulated if the transcript number was reduced by two and the adjusted p-value was $<0.1$ (figure 4.12 ). By building intersects of the downregulated genes I identified potential anterior and posterior target genes of the two pathways (figure 4.10). 

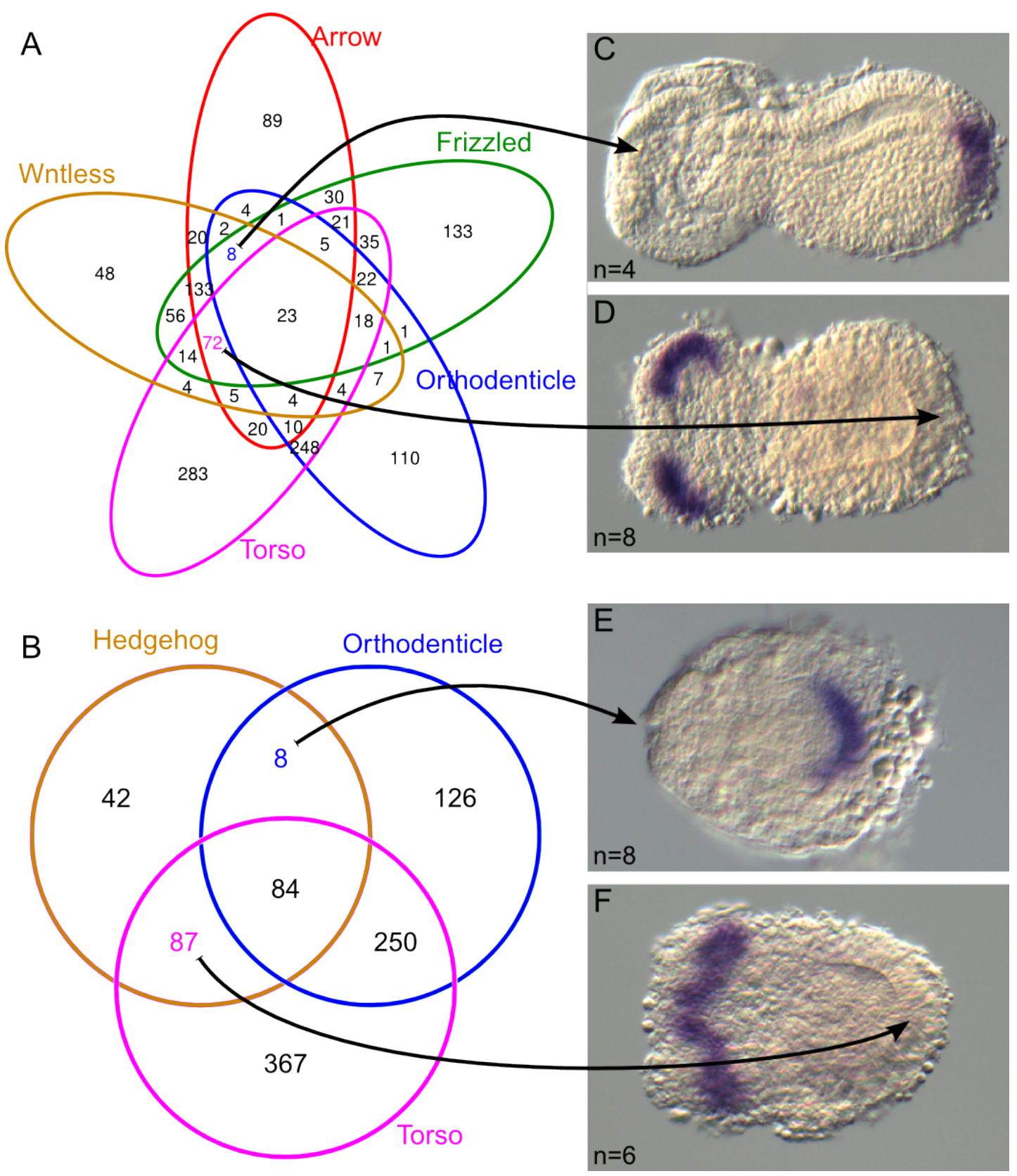

Figure 4.10: Subtractive RNAseq after RNAi - $(A, B)$ Venn diagrams show the number of downregulated genes in the different treatments and their intersects. $(C, D)$ Tc-wingless staining; $(E, F)$ Tc-hedgehog staining. In order to distinguish between anterior and posterior target gene sets, the head and growth zone expression domains were depleted by an independent treatment: In Tc-orthodenticle ${ }^{R N A i}$, the head domains were missing $(C, E)$ while in $T c$-torso ${ }^{R N A i}$ the growth zone domains were absent $(D, F)$. Blue numbers indicate the head specific target gene sets and red numbers the growth zone specific gene sets (see black arrows). 

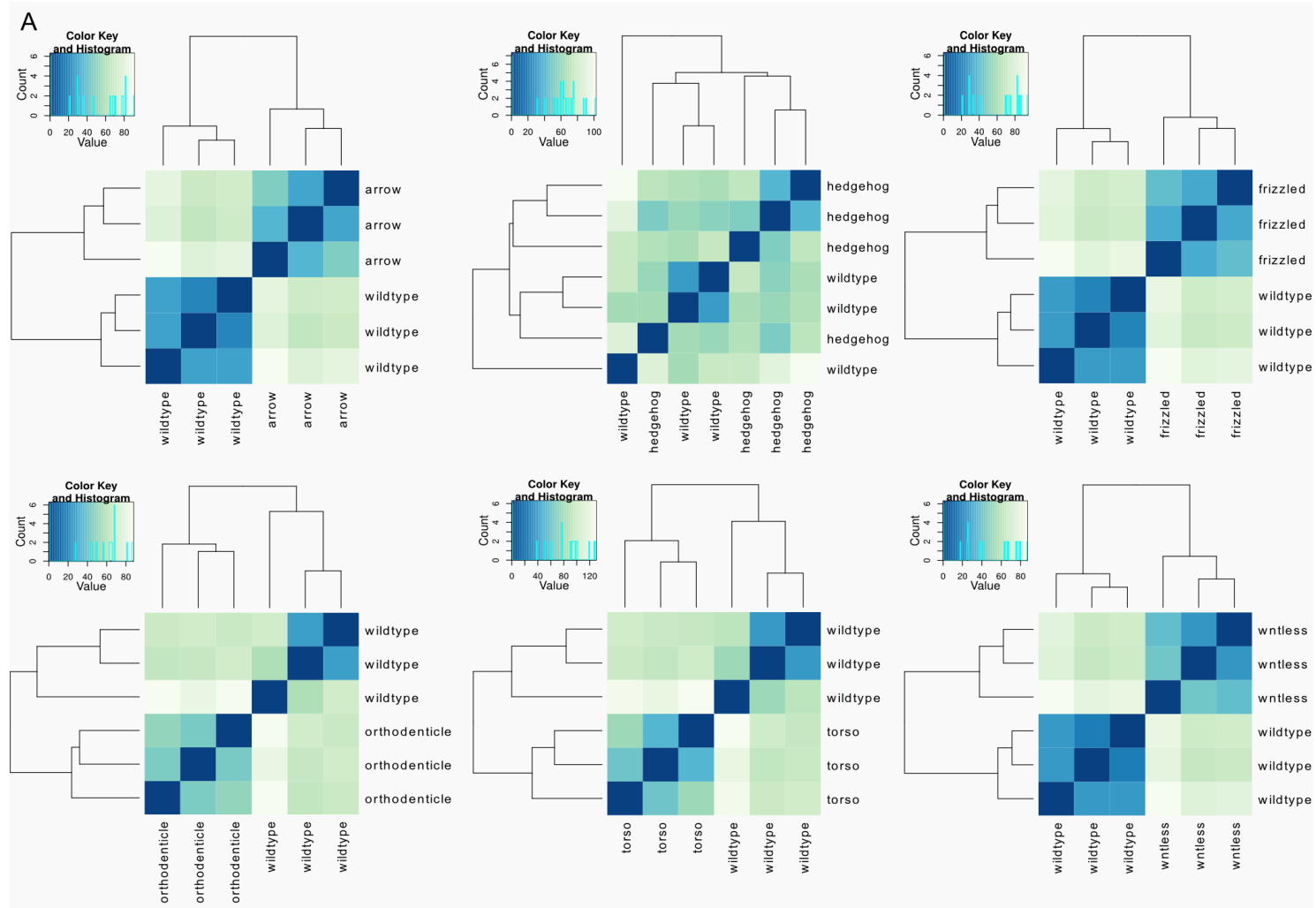

B
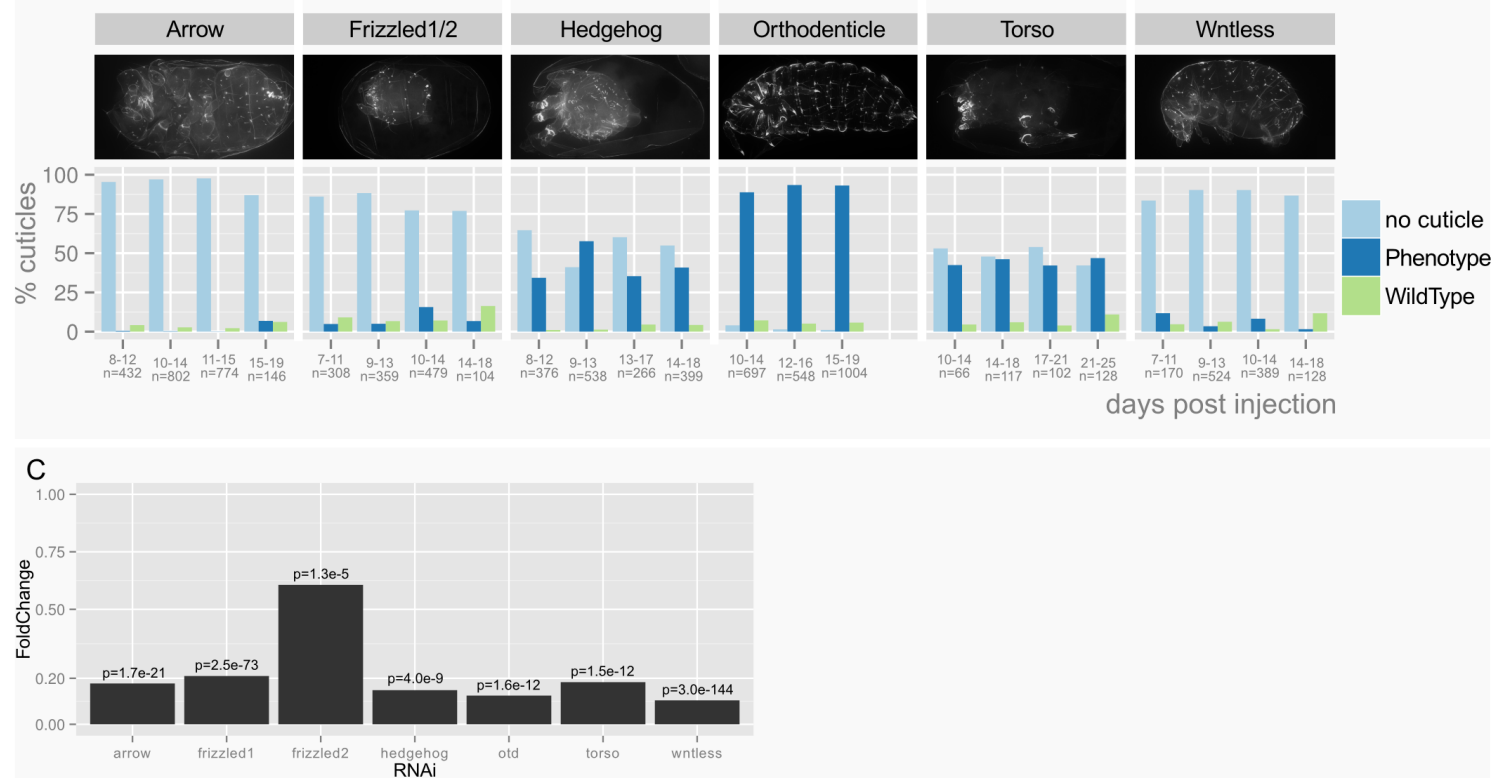


\subsection{RNAi-RNAseq reveals differences in anterior and posterior target gene sets of the Wnt and hedgehog pathways}

Figure 4.11 (preceding page): Quality controls of RNAseq experiment - (A) Cluster analysis of RNAseq treatments. All three replicates of the different treatments clustered together with the exception of the Tc-hedgehog treatment. The sequencing libraries of Tc-hedgehog were not prepared and sequenced in the same run resulting in a clustering of the sequencing runs instead of the treatment replicates. Analysis was performed with DESeq, heatmaps were plotted with gplots and RcolorBrewer. (B) Quantitative cuticular phenotype analysis of RNAseq treatments: As an additional control egg collections from the same dsRNA injections were set apart and the cuticular phenotypes of all treatments were analyzed quantitatively showing the high penetrance of those. The cuticle analysis was repeated on subsequent days showing the persistence of the RNAi effect. $(C)$ The RNAi treatments resulted in a transcript reduction of 80 to $90 \%$ for the single knock downs. In the double RNAi Tc-frizzled 1 was reduced by $80 \%$ and Tc-frizzled2 by $40 \%$. Barplots show fold changes of transcript levels compared to the wild type with Benjamini Hochberg adjusted p-values (false discovery rate)
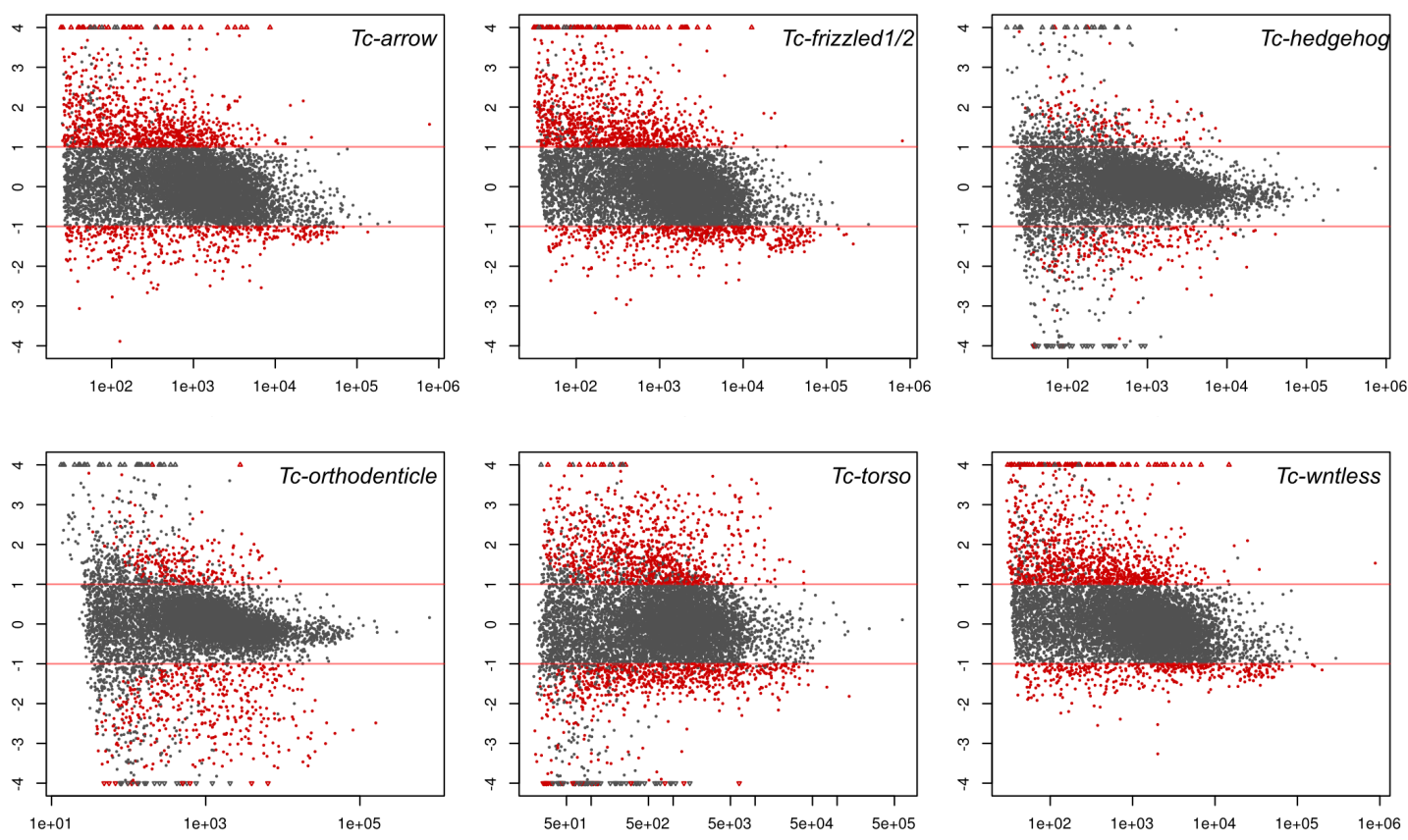

Figure 4.12: MA-plots of RNAseq treatments - Y-axis: $\log 2$ fold change values. X-axis: mean normalized counts of treatment and wild type samples for all genes. Significant differentially expressed genes (false discovery rate $<0.1$ ) are shown in red. 


\section{RESULTS}

\subsubsection{In situ hybridization of candidate gene sets}

In order to validate the candidate gene sets obtained from the RNAseq experiment I performed in situ hybridization for all transcription factors and signaling molecules and all genes without ortholog in Drosophila. Genes with low expression levels (below a normalized count of 200 in wild type) were excluded.

\subsubsection{Posterior Wnt targets}

The gene set contained 72 candidates (see figure 4.10). 22 of them were ribosomal genes. An additional seven genes (Tc-brachyenteron, Tc-caudal, Tc-twist, Tceven skipped, Tc-odd skipped, Tc-hairy, Tc-ladybird) were already known to be expressed in the growth zone in Tribolium. From the remaining 43 genes I selected 23 candidates for evaluation in the situ screen with the criteria described above. I obtained clones for 21 of them. These included seven genes with a Drosophila ortho- or homolog known to be involved in signaling or to be transcription factors. Six of these candidates showed specific posterior expression in the early germ band rudiment. From the remaining 14 genes without Drosophila homolog ten showed posterior expression. Three genes with highly similar sequences related to a retro transposon gave ubiquitous staining and one gene did not stain at all.

In summary the gene set contained

- 22 ribosomal genes

- 7 published genes showing posterior expression

- 21 selected candidates

- 7 with $D m$ homolog

* 6 showed posterior expression

- 14 without $D m$ homolog

* 10 showed posterior expression 


\subsection{RNAi-RNAseq reveals differences in anterior and posterior target gene sets of the Wnt and hedgehog pathways}

Of the 28 genes (including previously published genes) 23 showed posterior expression (82\%). See figure 4.13 for stainings and further details.

Several genes, not previously known to be active in the growth zone, were involved in signaling pathways: Tc-cAMP dependent PK1 (Hedgehog), elbowB (Notch), ETS-domain lacking and Misexpression suppressor of ras3 (Ras) [Baker et al., 2001, Bucher and Klingler, 2005, Huang and Rubin, 2000, Luque and Miln, 2007, Wang and Holmgren, 2000]. In line with the role of canonical Wnt signaling in the growth zone [Bolognesi et al., 2009, Beermann et al., 2011], important posterior patterning genes were included: Tc-caudal [Schulz et al., 1998, Copf et al., 2004] and the pair rule genes Tc-hairy, Tc-even skipped and Tc-odd skipped [Sommer and Tautz, 1993, Choe et al., 2006] in addition to the mesodermal genes Tc-twist [Sommer and Tautz, 1994] and Tc-ladybird [Cande et al., 2009, Jagla et al., 1997]. Unexpectedly for this early embryonic stage, I found two genes with a known function in Drosophila hind gut formation: Tc-brachyenteron and Tc-dichaete [Berns et al., 2008, Snchez-Soriano and Russell, 2000, Singer et al., 1996] and identified Tc-senseless as a novel player in gut development (see section 4.6 on page 46). GO enrichment analysis using the Drosophila annotations included processes like "gene expression" and "hindgut morphogenesis". The complete table including all enriched GO terms can be found in the appendix on page 88 .

\subsubsection{Anterior Wnt candidates}

This set contained eight genes. One was Tc-eyeless which was known to be expressed at the anterior. I selected six more for the in situ screen and obtained clones for five. Three had a Drosophila homolog. Tc-notum was expressed at the anterior in the germ band. Tc-adenosine 2 was expressed in the extra embryonic region at the anterior. Tc-amylase distal showed no expression. Of the unknown genes one was expressed in the anterior extra embryonic region and one in the anterior region of the germ band.

In total, I looked at six genes (one published, three with Dm homologs and two unknown). Three were localized in the anterior germ band and two in the anterior extra embryonic region. Taken together five of six genes were expressed in the anterior region of the embryo (83\%). See figure 4.13 for stainings and further 


\section{RESULTS}

details.

Apart from Tc-notum (a negative Wnt regulator Giraldez et al. [2002]) and Tceyeless [Yang et al., 2009, Posnien et al., 2011], not many target genes for the putative head signaling center were found.

\subsubsection{Posterior hedghehog candidates}

This gene set comprised 87 genes. Twelve genes with a Drosophila homolog and 15 without were selected for the in situ screen. I found that in the group of Drosophila homologs nine genes showed posterior expression compared to eight in the group without a Drosophila homolog. In total 17 of 27 candidates showed posterior expression in the in situ stainings (63\%). See figure 4.14 for stainings and further details.

In line with the overall correct germ band elongation in Tc-hedgehog ${ }^{R N A i}$ embryos ([Farzana and Brown, 2008] and figure $4.9 C_{2}$ ), I did not find segmentation genes apart from Tc-sloppy paired, which is a secondary pair rule gene in Tribolium [Choe and Brown, 2007]. I found four ortho/homologs of Drosophila genes involved in signaling across cell membranes: roadkill (hedgehog), cln3 (Notch, JNK), CG10960 (Jak/Stat) and spatzle (Toll) [Kent et al., 2006, Morisato and Anderson, 1994, Müller et al., 2005, Tuxworth et al., 2009]. Furthermore, I found eight ortho/homologs known to act as peptidases or peptidase inhibitors (CG5618, CG5639, CG32473, Jonah65Aiii, Puromycin sensitive peptidase, calpainB, Serpin 42Da, fat spondin) [Gelbart et al., 1997, Jekely and Friedrich, 1999]. GO term analysis using the Drosophila annotations as reference did not reveal any significantly enriched terms.

\subsubsection{Anterior hedgehog candidates}

This set contained eight genes. Three had a homologous gene in Drosophila. I selected seven for the in situ screen with only one (au2.g954) showing anterior expression at a later stage $(14 \%)$. 


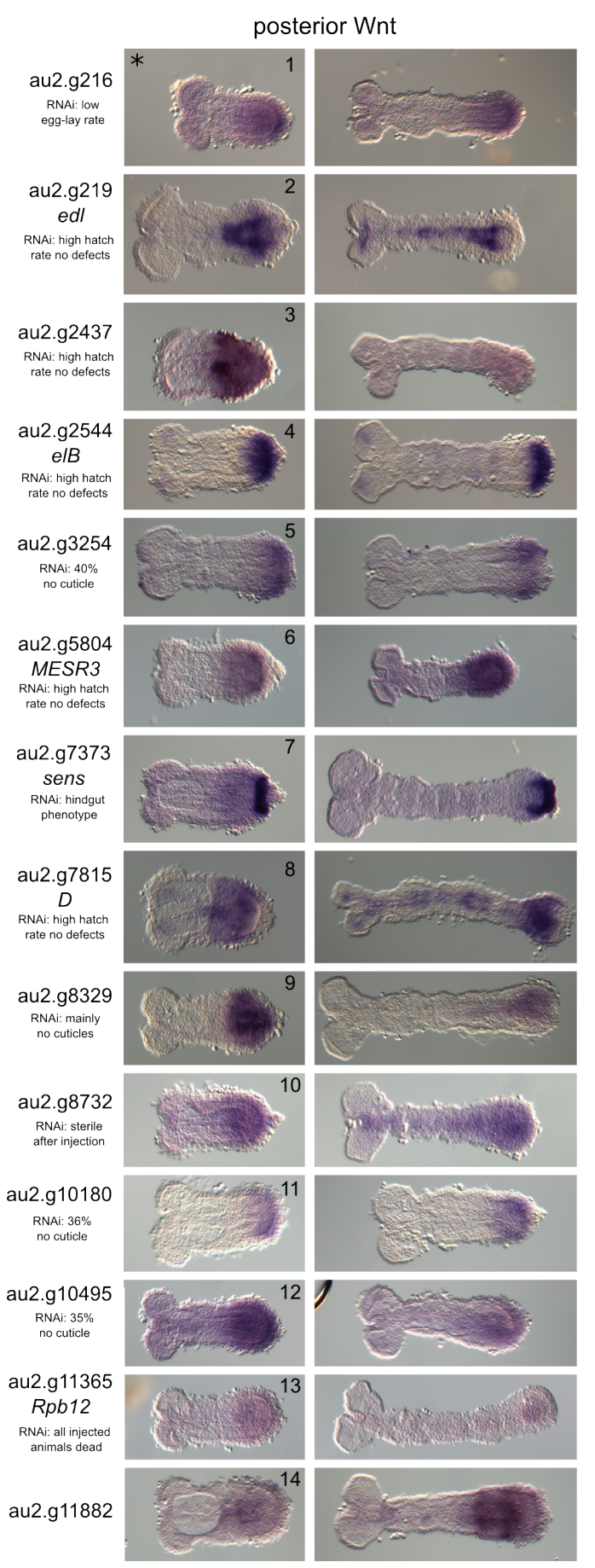

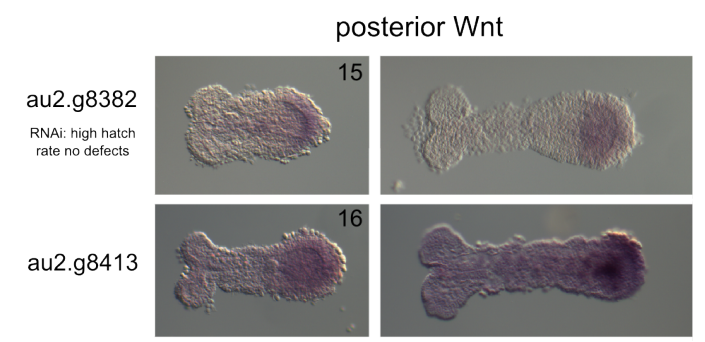
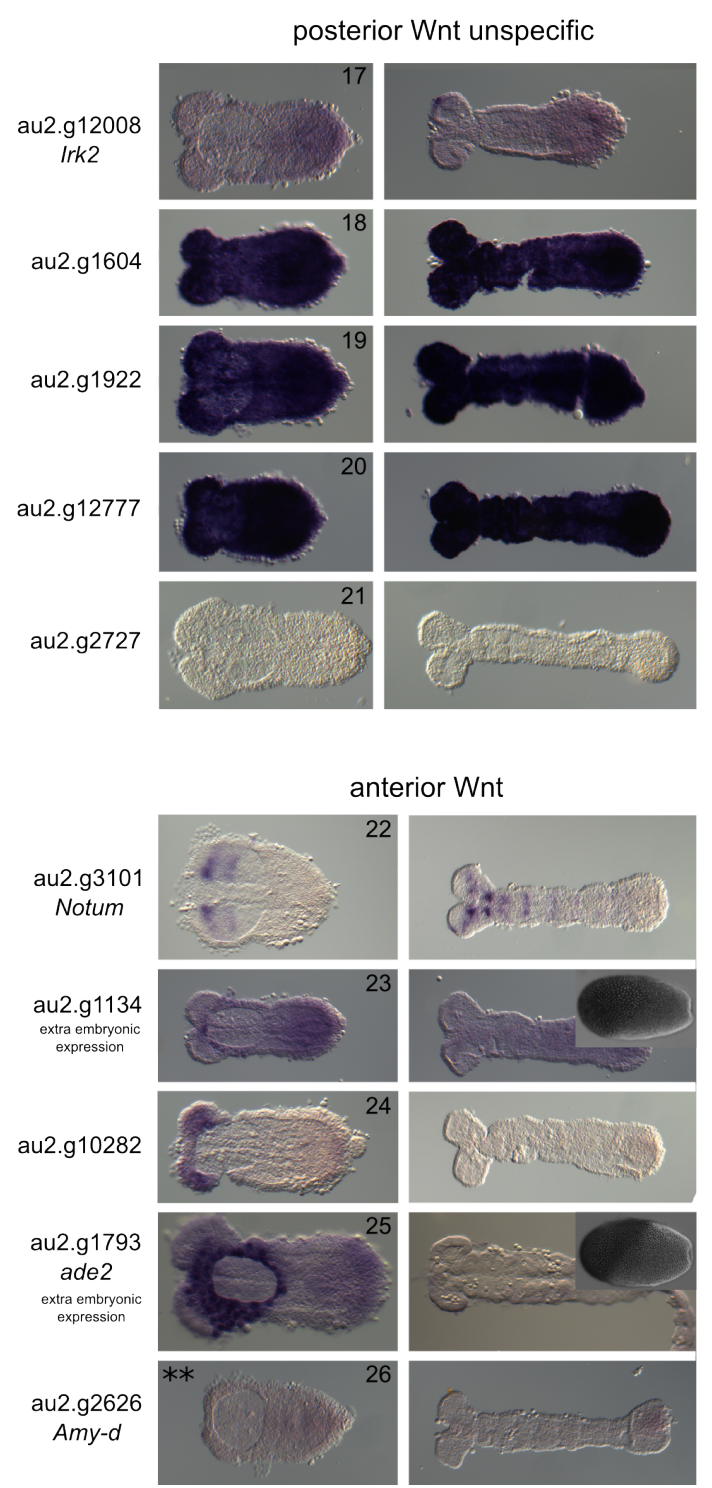
Figure 4.13 (preceding page): Posterior Wnt Candidates: - 23 candidate genes were selected for evaluation in the situ screen. I obtained clones for 21 of them. Those included seven genes with a Drosophila ortho- or homolog known to be involved in signaling and transcription factors. Six of these candidates showed specific posterior expression in the early germ band rudiment $(2,4,6,7,8,13)$. In addition 14 genes with no Drosophila homolog were added to the candidates for the in situ screen. 10 showed posterior expression $(1,3,5,9,10,11,12,14$, $15,16)$. Three genes with highly similar sequences related to a retro transposon gave ubiquitous staining $(18,19,20)$ and one gene did not stain at all $(21)$. Gene au2.g216 (*) was an overlapping candidate from both the Wnt and hedgehog gene sets.

Anterior Wnt candidates: This gene set contains 8 genes. I selected six for the in situ screen and obtained clones for five. Tc-notum (22) and au2.g10282 (24) were expressed exclusively in the head at this early stage. Tc-adenosine2 (25) and au2.g1134 (23) were expressed in the extra embryonic region at the anterior. Tcamylase distal showed no expression (26). Tc-eyeless was known to be expressed in the head. Tc-amylase distal $(* *)$ was an overlapping candidate from both the Wnt and hedgehog gene sets 
4.4 RNAi-RNAseq reveals differences in anterior and posterior target gene sets of the Wnt and hedgehog pathways
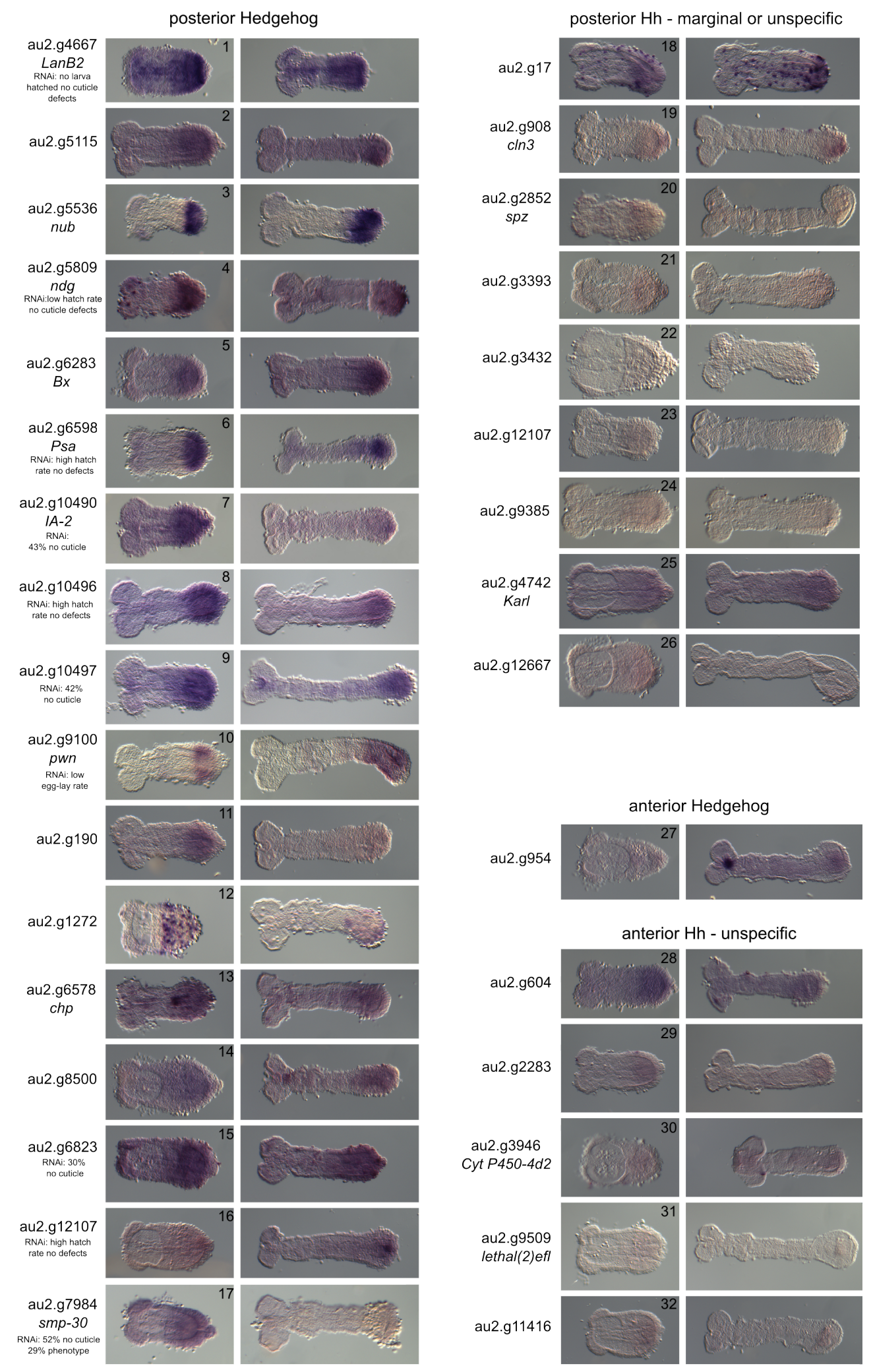
Figure 4.14 (preceding page): Posterior hedgehog candidates: - 27 genes were selected for the in situ screen including twelve with a homologous gene in Drosophila and 15 without. Among the genes with a homolog nine showed posterior expression $(1,3,4,5,6,7,10,13,17)$. In the group of unknown genes eight were expressed in posterior regions $(2,8,9,11,12,14,15,16)$.

Anterior hedgehog candidates: Eight genes were in the gene set. Seven genes were stained, with only one (27) showing anterior expression (14\%). One gene was a candidate in the anterior Wnt set and can be found there (figure 4.13). 


\subsubsection{Expression profiles of anterior expressed genes}

In a study previously conducted in our lab, the orthologs of vertebrate head patterning genes were analyzed ${ }^{1}$. Many of these genes were expressed at the ocular parasegment boundary at the germ rudiment stage. Surprisingly, only Tc-eyeless was among the downregulated genes in the anterior candidate sets (intersect of Tc-orthodenticle). Therefore, I checked how these potential anterior genes were regulated in the different RNAseq treatments. The selected genes included:

1. Genes with an exclusive anterior expression domain: Tc-cubitus interruptus, Tc-empty spiracles, Tc-eyeless, Tc-goosecoid, Tc-lim1, Tcorthodenticle, Tc-sloppy paired and Tc-twin of eyeless.

2. Genes with an anterior and posterior expression domain: $T c-$ hedgehog, Tc-tailless and Tc-wingless.
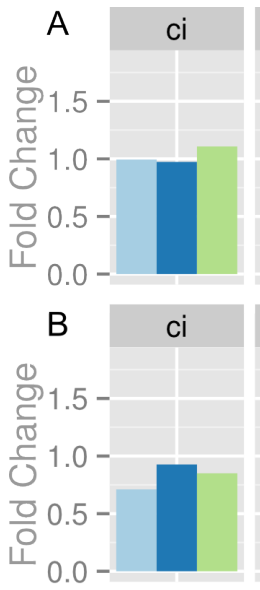

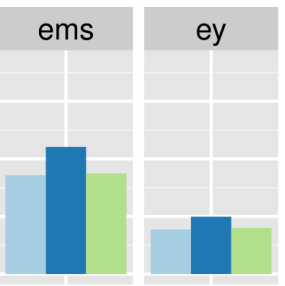

ems

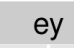

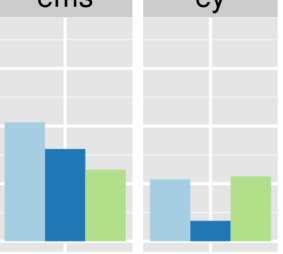
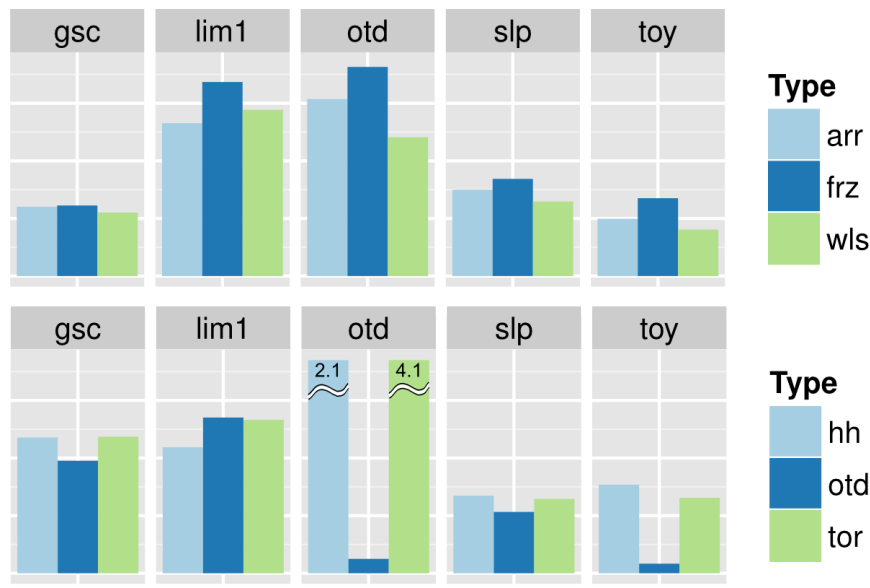

slp

toy
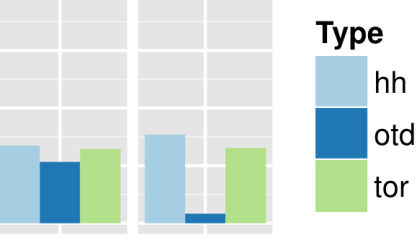

Figure 4.15: Fold change values of potential anterior target genes (A) Wnt treatments, Tc-arrow ${ }^{R N A i}, T c$-frizzled ${ }^{R N A i}$ and Tc-wntless ${ }^{R N A i}$. (B) Tchedgehog $^{R N A i}$, Tc-orthodenticle ${ }^{R N A i}$ and Tc-torso ${ }^{R N A i}$

${ }^{1} \mathrm{PhD}$ thesis Nico Posnien: Function and Evolution of highly conserved head genes in the red flour beetle Tribolium castaneum, Table 5.1 on page 82 and [Posnien et al., 2011] 


\section{RESULTS}

The candidate genes showed very similar expression profiles in the Wnt treatments, i.e. Tc-arrow ${ }^{R N A i}$, Tc-frizzled1/2/2NAi and Tc-wntless ${ }^{R N A i}$ (figure $4.15 \mathrm{~A}$ ). The expression levels of Tc-cubitus interruptus and Tc-empty spiracles were not affected. Tc-eyeless was highly reduced. Goosecoid was reduced by $40 \%$ and did therefore not pass the treshold of 50\%. Tc-lim1 and Tc-orthodenticle were upregulated. Tc-sloppy paired was only slightly downregulated. Tc-twin of eyeless was downregulated by more then $50 \%$ in $T c$-arrow ${ }^{R N A i}$ and $T c$-wntless ${ }^{R N A i}$ but not in Tc-frizzled1/2 ${ }^{R N A i}$.

In Tc-hedgehog ${ }^{R N A i}$ only Tc-eyeless was downregulated by $50 \%$. Tc-cubitus interruptus, Tc-sloppy paired and Tc-twin of eyeless were downregulated between $66 \%$ and $75 \%$. Tc-empty spiracles, Tc-goosecoid and Tc-lim1 were unaffected, whereas Tc-orthodenticle was upregulated by more then $100 \%$ (figure $4.15 \mathrm{~B}$, light blue bars).

In Tc-orthodenticle ${ }^{R N A i}$, Tc-eyeless and Tc-twin of eyeless were downregulated by over $75 \%$. Tc-sloppy paired was downregulated by $50 \%$, whereas the rest was unaffected (figure $4.15 \mathrm{~B}$, dark blue bars). In Tc-torso ${ }^{R N A i}$ four genes (Tc-empty spiracles, Tc-eyeless, Tc-sloppy paired and Tc-twin of eyeless) were downregulated by over $40 \%$ indicating that Tc-torso played un unexpected role in anterior patterning, which was also reflected by the over 4 -fold increase in Tc-orthodenticle transcript levels (4.15 $\mathrm{B}$, green bars).

The second group of candidate genes contained those with an anterior and posterior domain in the germ rudiment, i.e. Tc-hedgehog, Tc-tailless and Tc-wingless (figure 4.16). Tc-hedgehog was reduced by $40 \%$ in the Wnt treatments, corresponding to the loss of the posterior domain. In Tc-orthodenticle ${ }^{R N A i}$ it was reduced by $60 \%$ (loss of the anterior domains) and in Tc-torso ${ }^{R N A i}$ it was reduced by $50 \%$.

Tc-tailless was downregulated in all treatments with the exception of Tc-hedge$h o g^{R N A i}$. Therefore I could not assign it to the anterior or posterior gene set. Tc-wingless was downregulated between $70 \%$ and $50 \%$ in all treatments, again reflecting the loss of the anterior or posterior domain. 

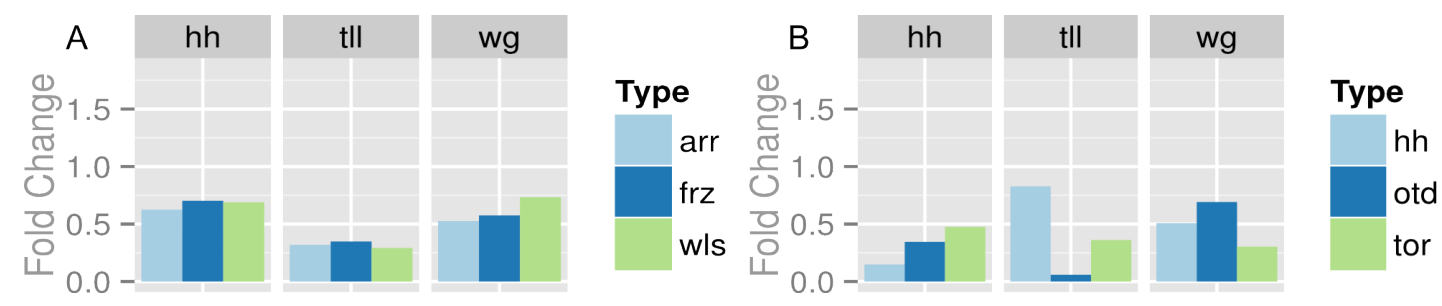

Figure 4.16: Fold change values of target genes with anterior and posterior expression domains - $(A)$ Wnt treatments, Tc-arrow ${ }^{R N A i}, T_{c}$-frizzled ${ }^{R N A i}$ and Tc-wntless ${ }^{R N A i}$. (B) Tc-hedgehog ${ }^{R N A i}$, Tc-orthodenticle $^{R N A i}$ and Tc-torso ${ }^{R N A i}$

\subsection{RNAi screen of selected candidates}

As many posterior segmentation and hind gut formation genes were comprised in the posterior gene sets, I searched for novel posterior patterning genes by knocking down selected genes specifically expressed in the growth zone, including 13 from the posterior Wnt targets, 10 from the posterior hedgehog targets and one gene found in both sets (figures 4.13 and 4.14 for stainings, figure 4.17 for quantification of cuticle phenotypes).

Eight genes led to significant empty egg phenotypes (>30\%, au2.g3254, au2.g6823, au2.g7815/ Dichaete, au2.g8329, au2.g10180, au2.g10490/ IA-2, au2.g10495, au2.g10497). Three genes led to low egg-lay rate or sterility (au2.g216, au2.g8732, au2.g9100/ pwn). Two RNAi's resulted in a very low hatch rate but without cuticle defects (au2.g4667/ LanB2, au2.g5809/ $n d g$ ), in one injection all animals died (au2.g11365/Rpb12) and one gene (au2.g7984/ smp-30) led to severe cuticle defects. Posterior segments were missing and the bristle pattern was disturbed in the entire larva (figure 4.18). 
A
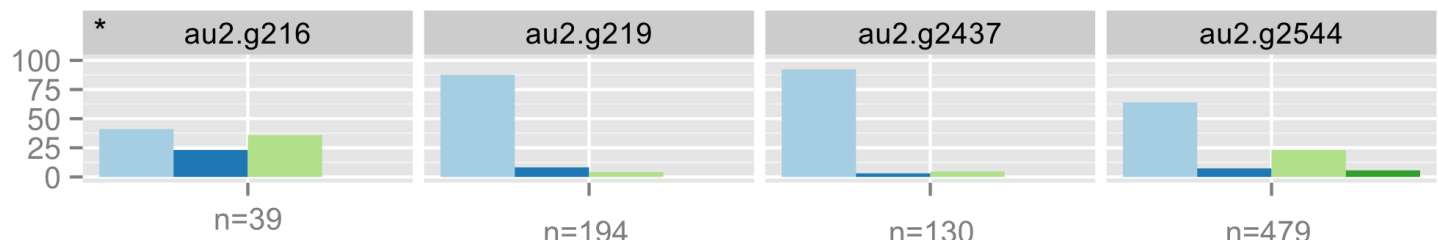

$\mathrm{n}=194$

$\mathrm{n}=130$

$n=479$
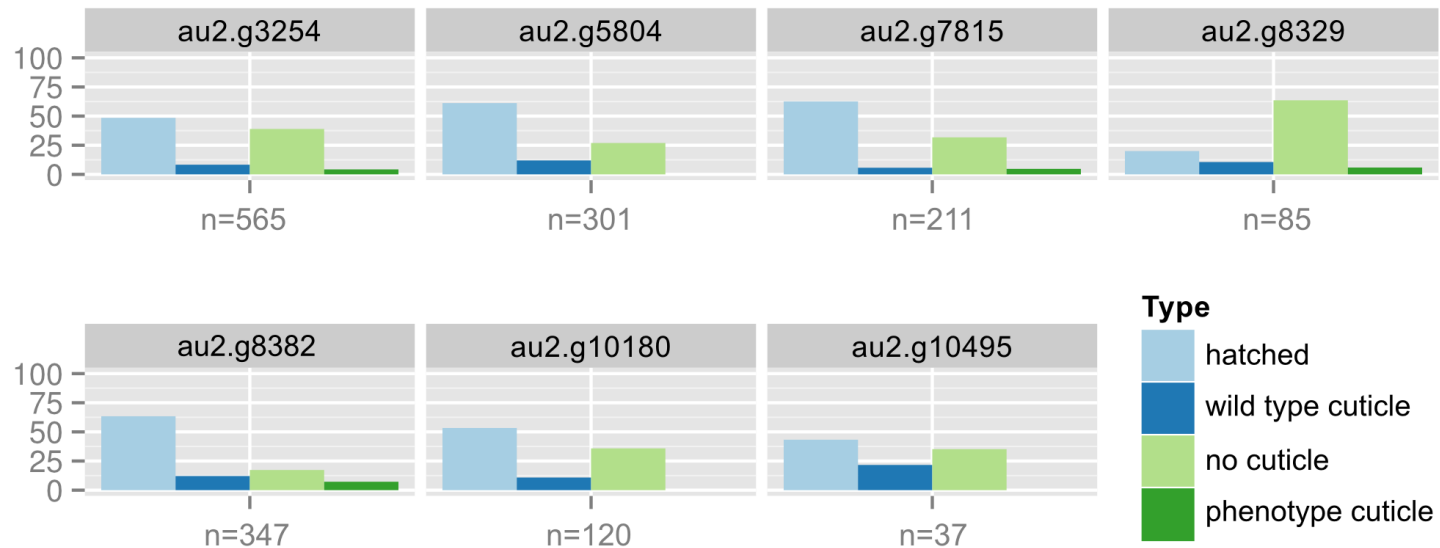

Type

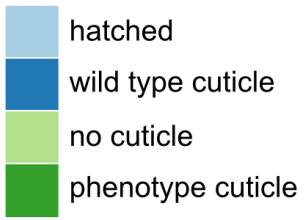

B

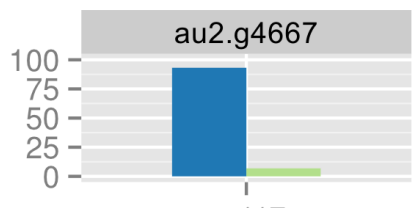

$\mathrm{n}=117$

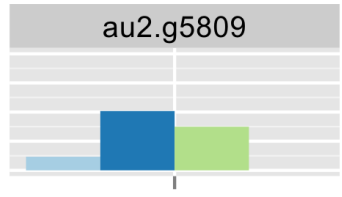

$\mathrm{n}=171$

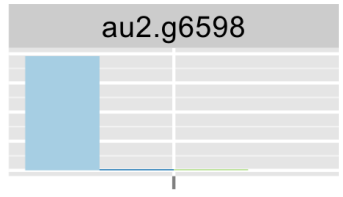

$\mathrm{n}=100$

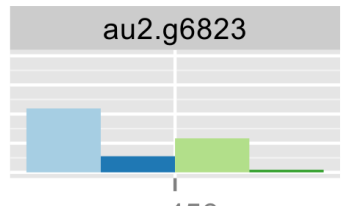

$n=456$
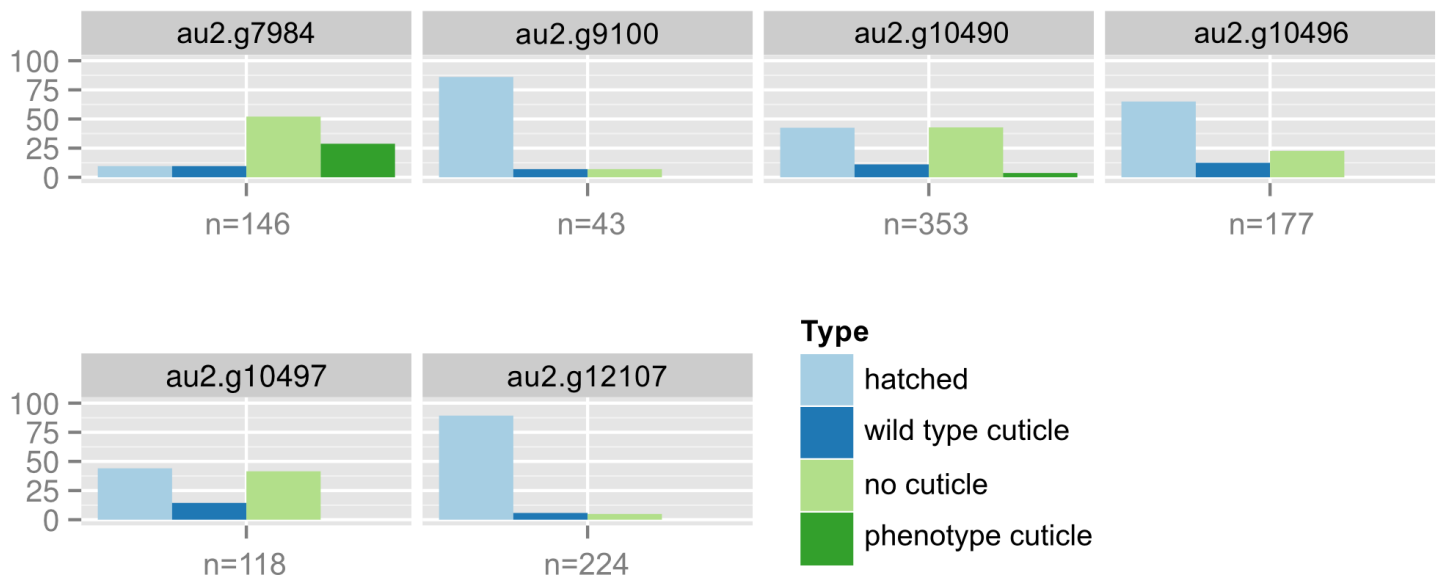

Type

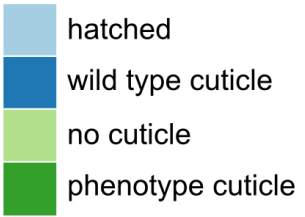




\section{Figure 4.17 (preceding page): Phenotype classes of RNAi screen: -} Barplots showing percentages of phenotype categories, i.e hatched, wild type cuticle (not hatched, no defects), no cuticle (empty egg) and phenotype cuticles. (A) Posterior Wnt candidates: 14 dsRNA injections were performed. One injection killed all animals (au2.g11365/Rpb12) and one injection led to sterility (au2.g8732). The phenotype of au2.g7373/sens is described in detail in figure 4.20. The remaining eleven genes were shown. Gene au2.g216 (*also candidate in hedgehog set) resulted in a low egg-lay rate. Genes au2.g3254, au2.g7815, au2.g8329, au2.g10180 and au2.g10495 showed a high percentage (¿30\%) of empty eggs (no cuticle produced). Only a very small percentage of cuticles showed severe but unspecific defects in various treatments (dark green bars) which were not considered to be significant. (B) Posterior hedgehog candidates: Ten dsRNA injections were performed. One additional gene was included in the Wnt and hedgehog sets (au2.g216, shown above). au2.g9100/pwn led to a reduced egg-lay rate. The genes au2. g6823, au2.g10490/IA-2 and au2.g10497 resulted in increased empty eggs as described above. The two candidates au2.g4667/LanB2 and au2.g5809/ndg resulted in very low hatch rates but no cuticular defects cold be detected. au2.g7984/smp-30 resulted in $50 \%$ empty eggs and $29 \%$ the phenotype shown in figure 4.18 .
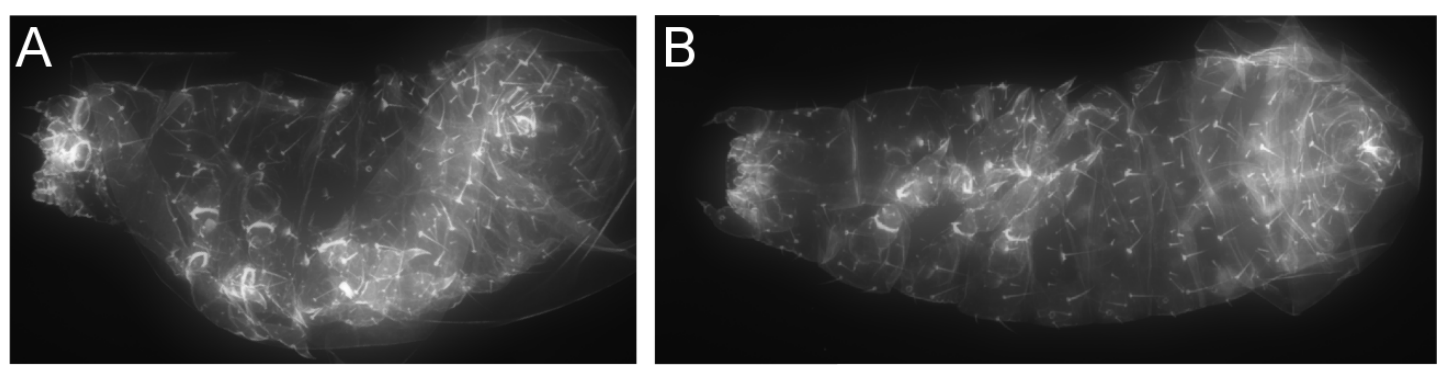

Figure 4.18: au2.g7984 dsRNA cuticle phenotype - $(A)$ Lateral and $(B)$ ventral views of RNAi cuticles showing the loss of posterior segments and misoriented bristles 


\section{RESULTS}

\subsection{A novel role for Tc-senseless in hindgut de- velopment in Tribolium}

Senseless, a Zn finger transcription factor, was first described in Drosophila where it plays a major role in peripheral nervous system development [Nolo et al., 2000]. Furthermore, it was shown that senseless was a target gene of wingless in the context of wing sensory organ development [Jafar-Nejad et al., 2006].
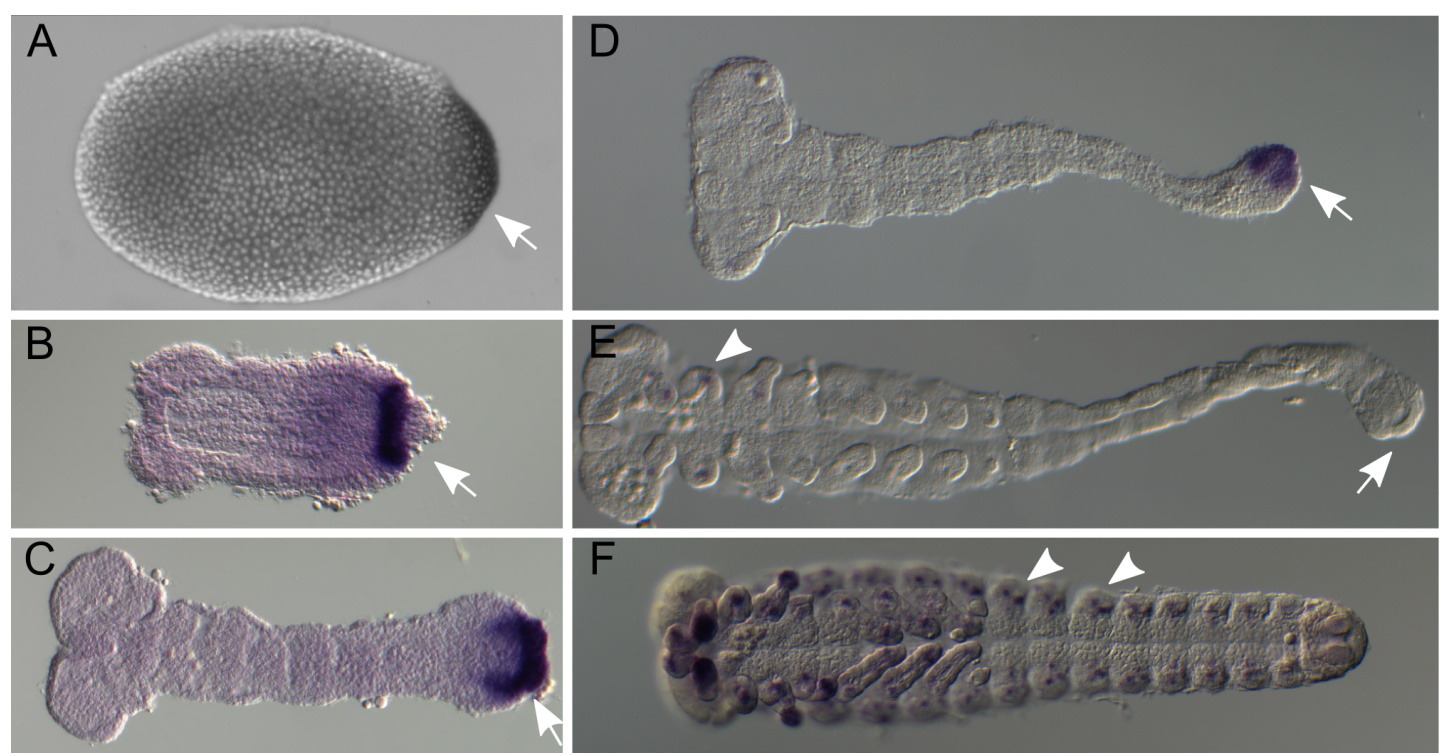

Figure 4.19: Expression of Tc-senseless - Developmental time series from undifferentiated blastoderms $(A)$ to retracting germbands $(F)$. Arrows mark growth zone expression, arrow heads mark lateral expression in the peripheral nervous system. Expression of Tc-senseless starts at the posterior pole in undifferentiated blastoderms $(A)$ and was maintained in the growthzone throughout elongation (arrows in $B-D$ ). During germ band retraction the posterior expression was lost (arrow in $E$ ) and arised de novo in putative PNS precursors (arrow heads in $E, F$ ). Pictures $B$ and $C$ are the same as in figure $4.13 \# 7$

In our RNAseq experiment Tc-senseless was a candidate in the posterior Wnt target gene set and was confirmed to be expressed in early germ rudiments at the posterior end (figure 4.13, gene au2.g7373). Tc-senseless expression started in blastoderms at the posterior pole. In early and elongating germ bands it was 
expressed in the growth zone. Later, during germ band retraction, expression was lost in the growth zone and started to arise in lateral spots, which likely corresponded to the expression in the peripheral nervous system known from Drosophila [Nolo et al., 2000] (see figure 4.19).

\subsubsection{Tc-senseless is required for hindgut development in Tribolium}

The first instar external larval cuticles did not show defects after adult RNAi but the hind gut cuticle was missing or highly reduced (figure $4.20 \mathrm{~B}, \mathrm{C}$ ). To exclude RNAi off-target effects, two non overlapping fragments of Tc-senseless dsRNA, were injected, with both reproducing the phenotype. $60 \%$ lacked the complete hindgut and $15 \%$ showed reduced hindgut structures with dsRNA fragment 1. The penetrance was slightly weaker for fragment 2 with only $50 \%$ missing the hindgut but over $20 \%$ having reduced hindgut structures (figure $4.20 \mathrm{D}$ ).

The hindgut becomes visible only much later during development. Therefore, I wondered whether T-senseless was required early (figure $4.19 \mathrm{~A}, \mathrm{~B}$ ) or at later elongating stages (figure $4.19 \mathrm{C}$ ). To confirm that the gut phenotype was due to the early expression in the growth zone, staggered embryonic RNAi ${ }^{1}$ was performed. Tc-senseless dsRNA was injected in 4-7 hour (early blastoderm) and in 14-15 hour (elongating germ band) embryos. About $60 \%$ of the early injected eggs did not develop a cuticle in Tc-senseless ${ }^{e R N A i}$ compared to $50 \%$ in the injection buffer control. Another $10 \%$ of injected eggs showed severe cuticular defects, both in Tc-senseless ${ }^{e R N A i}$ and the injection buffer control. In the later time point injections the percentages of empty (no cuticle) and severly defected cuticles were slightly lower but again comparable between Tc-senseless ${ }^{e R N A i}$ and injection buffer controls. As shown previously, this makes it likely that these effects were due to the injection treatment and not the RNAi [Grossmann et al., 2009].

\footnotetext{
${ }^{1}$ embryonic injections were carried out by Daniela Grossmann
} 


\section{RESULTS}
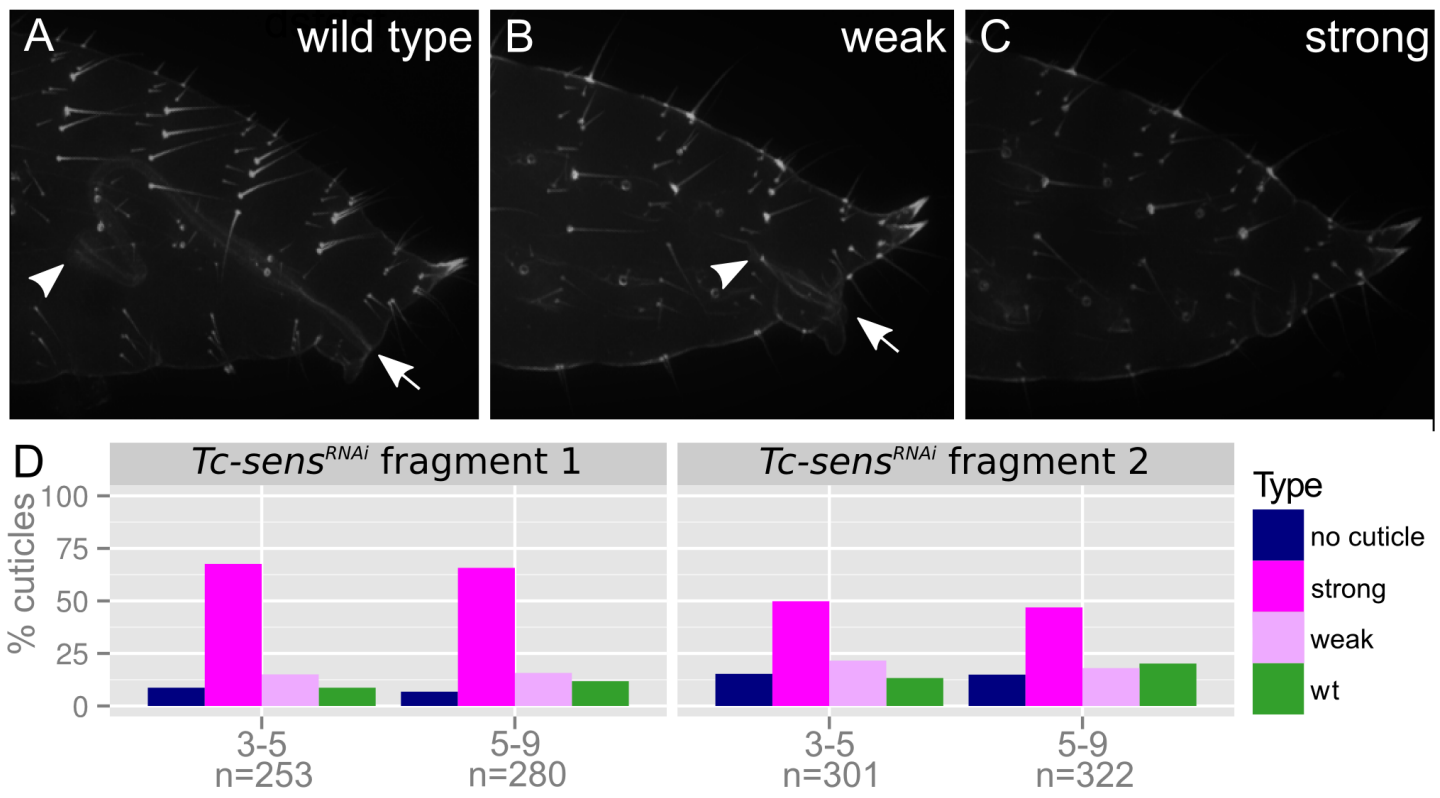

fragment 2

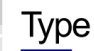

ype

no cuticle trong weak wt
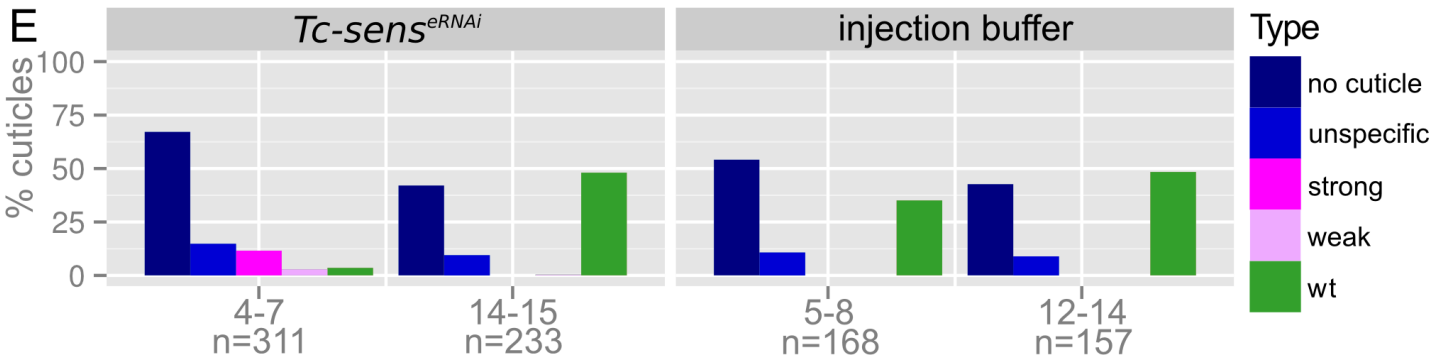

injection timepoint in hours after egg lay

Figure 4.20: Tc-senseless ${ }^{R N A i}-(A-C)$ LSM images: arrow and arrowhead mark extremes of the hind gut. $(D)$ Phenotype penetrance in non overlapping dsRNA fragments. (E) Staggered embryonic RNAi. (A) Wild type hind gut, extending in length over four segments. Weak phenotypes $(B)$ show a shortened hindgut (compare arrows with wild type in $A$ ) while strong phenotypes lack the hindgut altogether $(C)$. $(D)$ Cuticles showing the phenotype on different days post dsRNA injection in adults. au2.g7373_F1 and au2.g7373_F2 were non overlapping dsRNA fragments of Tc-senseless with both reproducing the phenotype. $(E)$ In staggered embryonic RNAi the phenotype could only be reproduced in early injected eggs (4-7 hour old). Later injection (14-15 h) did not show the hindgut phenotype, indicating an early essential function of Tc-senseless in the growth zone. 
Only injections in early blastoderm stages (4-7h, figure 4.19 A) interfered with gut formation ( $11.6 \%$ strong phenotype, $3.6 \%$ weak phenotype), while injection in elongating embryos (14-15h, similar to stage in figure $4.19 \mathrm{C}$ ) did not elicit the gut phenotype (no strong phenotype detected, $0.4 \%$ or one cuticle showed the weak phenotype. Hence, Tc-senseless was required early in the growth zone for hind gut development. 
4. RESULTS 


\section{5}

\section{Discussion}

\subsection{Dynamic expression patterns in the estab- lishment of the ocular and antennal paraseg- ment boundaries}

Using high resolution fluorescent in situ hybridization I observed a dynamic expression pattern of Tc-hedgehog in the head. Expression starts at the ocular parasegment boundary, extends backwards to split up and gives rise to the antennal domain. Only later, during development Tc-wingless arises at the antennal boundary to probably stabilize the parsegmental border. I analyzed two candidate genes (knirps and empty spiracles) known to interfere with antennal development and studied the effects of these candidates on the segment polarity genes wingless and hedgehog.

Based on the following observations I propose a genetic model that could explain this segmentation process:

Tc-orthodenticle activates Tc-hedgehog at the ocular parasegment boundary. In Tc-orthodenticle ${ }^{R N A i}$ ocular Tc-hedgehogis lost (figure 4.10). In Tc-hedgehog ${ }^{R N A i}$ ocular Tc-wingless is lost, but in Tc-arrow ${ }^{R N A i}$ Tc-hedgehog is unaffected initially and fades only later (figure 4.9). This puts Tc-hedgehog between Tc-orthodenticle and Tc-wingless in the activation cascade. 


\section{DISCUSSION}

Tc-knirps activates Tc-hedgehog posterior of the ocular parasegment boundary. In Tc-knirps ${ }^{R N A i}$ antennal Tc-hedgehog never arises, whereas ocular Tc-hedgehog is unaffected ([Cerny et al., 2008, Peel et al., 2013] and figure 4.3).

Tc-empty spiracles represses Tc-wingless. Tc-empty spiracles is expressed between the ocular and antennal Tc-wingless domains and Tc-empty spiracles ${ }^{R N A i}$ leads to ectopic Tc-wingless expression in this region ([Schinko et al., 2008] and figure 4.6).

Convergent extension is the driving force in separating ocular and antennal Tc-hedgehog. This was shown in spiders where a similar patterning process occurs [Kanayama et al., 2011]. In Tribolium, the early germ rudiment becomes longer and narrower during development hinting at a similar process (figure 4.4). In addition, immunohistochemistry stainings for the cell division marker Phosphohistone-H3 (PH3) showed no significant amount of cell division during this stages ${ }^{1}$.

A negative self-regulatory loop splits the Tc-hedgehog domain. In $T c$ smoothened $^{R N A i}$ and Tc-cubitus interruptus ${ }^{R N A i}$ the anterior Tc-hedgehog domain is broadened but fails to split (figure $4.2 B$ and $4.9 C_{3}, C_{4}$ ).

\subsubsection{Genetic model for the patterning of the antennal segment}

Here I propose a new model for Tribolium anterior head patterning, adding some key genes and interactions, starting with the initial activation of hedgehog via orthodenticle at the ocular parasement boundary.

\section{Establishment of the ocular parasegment boundary}

Tc-hedgehog has an anterior expression domain in the blastoderm, whereas Tcwingless is expressed only in the growth zone before germ band formation [Farzana and Brown, 2008, Bolognesi et al., 2008]. Together with the loss of ocular

\footnotetext{
${ }^{1}$ data not shown and $\mathrm{PhD}$ thesis Sebastian Kittelmann, page 39-40, although n was only 2
} 
Tc-wingless in Tc-hedgehog ${ }^{R N A i}$ (figure $4.9 C_{1}$ ) the establishment of the ocular parasegment can be summarized in two steps:

1. Tc-orthodenticle activates Tc-hedgehog

2. Tc-hedgehog activates Tc-wingless

Now the parasegment boundary is stabilized via the cross-regulatory interactions as in the trunk, indicated by the loss of ocular Tc-hedgehog in Tc-arrow ${ }^{R N A i}$ later during development (figure $4.9 B_{4}$ ).

\section{Backwards expansion of Tc-hedgehog}

In Tc-knirps ${ }^{R N A i}$ ocular Tc-hedgehog is unaffected, while the antennal domain does not develop ([Cerny et al., 2008] and figure 4.3). Therefore I assume that Tc-knirps activates Tc-hedgehog in all cells posterior to the ocular domain. Double in situ stainings with Tc-knirps and Tc-wingless show that they are expressed adjacently before the backwards expansion of Tc-hedgehog starts. As the hedgehog domain extends backwards, the gap between Tc-wingless and Tc-knirps increases (figure 4.4). Convergent extension is probably the driving force that increases this gap. Cell tracking in in vivo imaging could show that this is the case.

Tc-hedgehog cannot activate Tc-wingless anterior to it because of the repressing function of Tc-empty spiracles on Tc-wingless. In Tc-empty spiracles ${ }^{R N A i}$ all cells become $T c$-wingless positive in the antennal segment. Once the moving Tc-hedgehog domain is beyond the Tc-empty spiracles domain it succeeds in activating $T c$-wingless and by doing so the antennal parasegment boundary is established and stabilized. In support of this mechanism in Tc-hedgehog ${ }^{R N A i}$ antennal Tc-wingless is lost (figure $4.9 C_{2}$ ).

\section{Splitting of Tc-hedgehog}

In Tc-smoothened ${ }^{R N A i}$ the Tc-hedgehog domain moves backwards but stays attached to the ocular domain. The result is a single broad Tc-hedgehog domain that fails to split up (figure $4.9 C_{3}, C_{4}$ ). This leads to the assumption that a functionial hedgehog pathway is essential for the Tc-hedgehog domain splitting. However, in Tc-cubitus interruptus ${ }^{R N A i}$ the splitting process is unaffected. Because of the dual role of cubitus interruptus as repressor and activator [Aza-Blanc 


\section{DISCUSSION}

et al., 1997] the interpretation of these effects is difficult.

\section{Stopping the backwards movement of Tc-hedgehog}

No experimental data altering the position of antennal Tc-hedgehog has been obtained. However, one simple hypothesis could be that once Tc-knirps enters into the field where pair-rule genes are expressed, the activating properties of $T c$ knirps on Tc-hedgehog are overridden by them. In RNAi embryos against different pair-rule genes antennal Tc-engrailed seems to be affected in some cases [Choe et al., 2006]. Antennal Tc-engrailed is expressed relatively late during development which would make Tc-hedgehog a better read out to test this assumptions. In addition in Tc-even skipped ${ }^{R N A i}$ the Tc-knirps domain is expanded into more posterior regions [Peel et al., 2013].

Previous findings showed that a similar process occurs during anterior segment patterning in spiders [Pechmann et al., 2009]. Orthodenticle is the key factor activating hedgehog in the anterior head. A model involving an autoregulatory signaling network was proposed. The key players involved were hedgehog, cubitus interruptus, orthodenticle and odd-paired. The model implies short- and long range morphogen activities of hedgehog and an unknown mechanism for the stripe splitting [Kanayama et al., 2011].

\subsubsection{Outlook stripe splitting project}

Some key experiments are still missing to complete this project. Those include:

- In vivo imaging: In spiders, the backwards movement of hedgehog is explained by the traveling of hedgehog expression across a field of cells in a wave-like manner. This would include that single cells have to turn the expression of hedgehog on and off. Another approach to describe the phenomenon would be that there is no dynamic expression of hedgehog. Instead the movement of hedgehog is due to the influx of lateral cells, i.e. convergent extension. A live imaging movie with a nuclear $\mathrm{GFP}^{1}$ line could

\footnotetext{
${ }^{1} \mathrm{GFP}$ - green fluorescent protein
} 


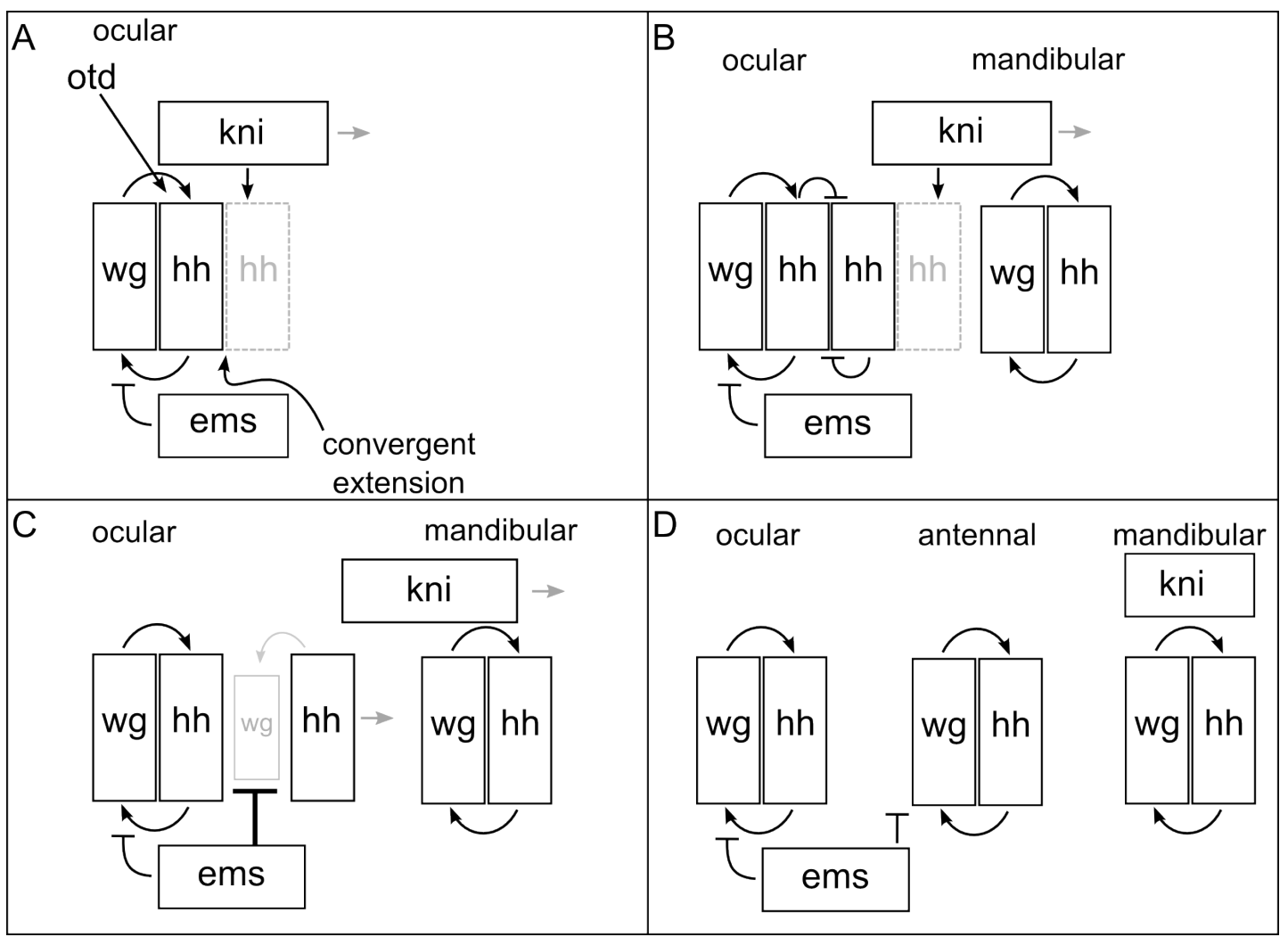

Figure 5.1: Genetic model to establish the antennal parasegment boundary in Tribolium - (A) Tc-orthodenticle activates ocular Tc-hedgehog. Ocular Tc-hedgehog activates Tc-wingless. Once established, both segment polarity pathways stabilize the boundary. Tc-knirps, initially expressed adjacent to Tc-wingless, is separated and pushed towards the posterior pole (convergent extension), activating Tc-hedgehog in the process. (B) The broadening Tc-hedgehog domain initiates a negative auto-regulatory loop of the hedgehog pathway, which leads to the stripe splitting. $(C)$ During its backwards expansion and splitting, Tc-hedgehog cannot activate Tc-wingless due to the repressive effect of Tc-empty spiracles in this field. (D) Once Tc-hedgehog moves clear of the Tc-empty spiracles expression domain, it activates Tc-wingless and stabilizes the antennal segment. The activating function of Tc-knirps during backwards traveling has come to a halt at the mandibular segment. The pair-rule gene circuit is active there and governs patterning in classic mode. 
be used to show that such processes occur in the early Tribolium germ rudiment.

- In situ stainings: To refine the expression topologies of the key genetic factors in the model, some double in situ stainings have to be done, including:

- Tc-hedgehog - Tc-knirps to show co-expression of the two genes.

- Tc-empty spiracles - Tc-knirps to show initial co-expression and later adjacent expression.

- RNAi experiments: Since it is unclear how the posterior (antennal) Tchedgehog domain comes to a halt, RNAi against some candidate genes could be used to modify the location of these boundary. Candidate genes include: pair rule genes and genes with an expression domain at the posterior border of antennal Tc-hedgehog, e.g. Tc-labial

\subsection{RNAseq after RNAi reveals differences in anterior and posterior target gene sets}

\subsubsection{Segment polarity interactions in head and growth zone}

This work identifies the first comprehensive target gene sets of the hedgehog and Wnt pathways in Tribolium embryogenesis using RNAseq. Further, I found a complementary cross regulation of Wnt and hedgehog pathways in head and trunk: hedgehog signaling acts upstream of Tc-wingless in the head but has no influence on posterior Tc-wingless expression (figure $4.9 C_{1}, C_{2}$ ). In the growth zone Wnt signaling acts upstream of Tc-hedgehog and is also needed for Tc-wingless initiation while hedgehog signaling has no influence on Tc-wingless expression. Apparently, the canonical mutual activation between these pathways is restricted to the trunk parasegment boundaries not only in Drosophila [Gallitano-Mendel and Finkelstein, 1997] but also in Tribolium [Oppenheimer et al., 1999]. 


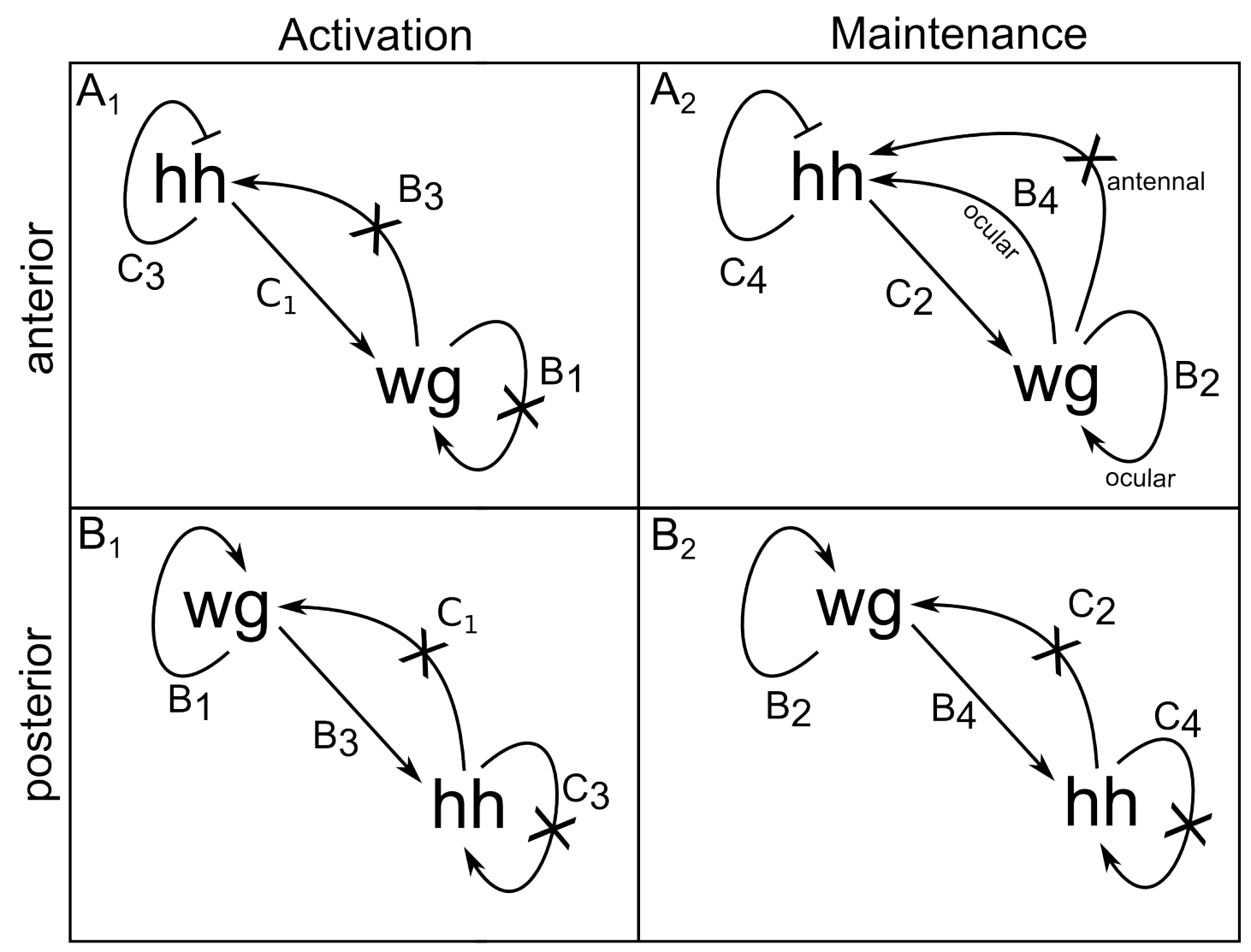

Figure 5.2: Segment polarity interactions in head and growth zone $\left(A_{1}, A_{2}\right)$ Anterior; $\left(B_{1}, B_{2}\right)$ Posterior; arrows indicate activation, crossed arrows non activation, dotted arrows mark ambiguous interactions. Arrows are labeled with the in situ from which the respective interaction was deduced (see figure 4.9. $\left(A_{1}\right)$ Anterior activation: Hedgehog pathway acts upstream of Tc-wingless. Hedghog pathway represses Tc-hedghog expression during backwards traveling. Wnt pathway has no effect on Tc-wingless and Tc-hedgehog expression. $\left(A_{2}\right)$ : Hedgehog pathway acts upstream of Tc-wingless expression at the ocular and antennal parasegment boundaries. Hedghog pathway represses Tc-hedghog expression during backwards traveling. Wnt pathway has no effect on antennal Tc-hedgehog but is needed for maintenance of ocular Tc-hedgehog. With a disrupted Wnt pathway ocular Tc-wingless is lost which could indicate autoregulation (dotted arrow). However, mutual activation between the two pathways is also possible and more likely. $\left(B_{1}\right)$ Posterior activation: The Wnt pathway is needed for Tc-wingless (auto regulation) and Tc-hedgehog activation. The hedgehog pathway has no effect on Tc-wingless or Tc-hedgehog expression. $\left(B_{2}\right)$ Posterior Maintenance: The interactions are the same as in posterior activation. 


\section{DISCUSSION}

\subsubsection{The ocular parasegment boundary as anterior sig- naling center}

Based on the expression of their ligands, both Wnt and hedgehog signaling pathways were likely to play crucial roles in early head and growth zone patterning. Unexpectedly, I did not find many targets in the head (eight compared to 72/87 in the growth zone) domain, although this domain corresponds to the vertebrate mid-hind-brain boundary and several putative target genes start being expressed there [Posnien et al., 2011]. By looking at the expression levels in the single treatments I showed that most of these anterior target genes are indeed downregulated in Tc-orthodenticle ${ }^{R N A i}$. In the Wnt and hedgehog treatments many of the anterior candidates were downregulated, however, they did not show in our intersects because the tresholds of reduction by half was not met in all treatments. Tcgoosecoid, a gene involved in stomatogastric nervous system development in the foregut in Drosophila [Hahn and Jäckle, 1996] was downregulated by only $40 \%$ in the Wnt and hedgehog treatments. Tc-empty spiracles was not affected by the treatments which is not surprising if it is considered a head-gap gene lying upstream of segment boundary formation [Cohen and Jürgens, 1990, Schinko et al., 2008]. Tc-tailless [Weigel et al., 1990, Schroder et al., 2000] was downregulated in all Wnt treatments, in Tc-orthodenticle ${ }^{R N A i}$ but also in Tc-torso ${ }^{R N A i}$. Hence, it was not possible to distinguish between anterior and posterior in this case. Tchedgehog was downregulated by $40 \%$ in the Wnt treatments in correlation with the loss of Tc-hedgehog expression in the growth zone. In Tc-orthodenticle ${ }^{R N A i}$ it was reduced by $60 \%$ and in Tc-torso ${ }^{R N A i}$ by $50 \%$ corresponding to loss of the anterior or posterior domain. Tc-orthodenticle was upregulated in the Wnt and hedgehog treatments. Probably the ocular parasegment boundary can be interpreted as a barrier with repressive functions, constricting Tc-orthodenticle to the anterior most part of the head. Tc-eyeless and Tc-twin of eyeless [Yang et al., 2009] were both downregulated in the Wnt and hedgehog treatments making these two genes good downstream targets of the two pathways. Tc-wingless was reduced by almost $50 \%$ in Tc-arrow ${ }^{R N A i}$, Tc-frizzled ${ }^{R N A i}$ and Tc-hedgehog ${ }^{R N A i}$ in line with the loss of the growth zone or ocular domain.

Another possible explanation for the low number of anterior targets could be the 
chosen developmental stage. Since the anterior head segments are not yet established, the segment polarity genes might still be involved with patterning and positioning processes (see section 4.1) and not in the activation of target genes. RNAseq at a later developmental stage could show if this is the case.

The large increase in Tc-orthodenticle transcripts in Tc-torso ${ }^{R N A i}$ (over 4-fold) showed that Tc-torso had an unexpected effect on anterior development. Due to this fact, some of the anterior target genes could be in the intersect of $T c$ orthodenticle and Tc-torso. Hence, these genes were not found in the RNAseq approach.

One interesting hypothesis to test is that the ocular parsegment boundary represents a barrier with repressive properties instead of being a signaling center activating target genes.

\subsubsection{Posterior target genes}

A similar number of genes are regulated by hedgehog and Wnt signaling in the growth zone and the gene sets are quite different from each other. An intriguing feature of the hedgehog dataset is the large number of genes potentially involved in a proteolytic cascade including the Toll ligand Tc-spatzle. Based on this and on the fact that posterior segmentation is unaffected in Tc-hedgehogRNA $A^{R N A i}$ [Farzana and Brown, 2008] a role in ongoing non-ectodermal patterning in the growth zone could be hypothesized, where mesoderm and neuroectoderm probably need to be specified continuously [Handel et al., 2005].

The finding of several crucial posterior patterning genes in the growth zone Wnt set was not unexpected, given the conserved role of Wnt signaling in posterior patterning in bilaterians [Martin and Kimelman, 2009] and even pre-bilatarians [Petersen and Reddien, 2009]. However, the large number of ribosomal genes indicates that Wnt signaling does not only govern pattern formation but also ensures that the respective cells are metabolically prepared for growth. Recently it was shown that pygo, a transcriptional co-activator of armadillo/ $\beta$-catenin $[$ Kramps et al., 2002], is involved in ribosome biogenesis in human cancer cell lines [Andrews et al., 2013]. Taken together, this could indicate that a link between the Wnt pathway and ribosome metabolism may be a more general feature. 


\section{DISCUSSION}

Finally, I found several hindgut genes to be controlled by Wnt signaling [Lengyel and Iwaki, 2002], and identified Tc-senseless as novel gene involved in hindgut development. It is required early in the growth zone despite the fact that the hindgut develops only hours later. Apparently, there is molecular specification of posterior terminal cells before completion of abdominal segmentation. This function is different from the Drosophila ortholog Dm-senseless, which is not expressed in the hindgut but in sensory organ precursors and is required for their formation [Nolo et al., 2000]. Interestingly, the paralog Dm-senseless-2 is expressed in the anterior midgut in Drosophila but a phenotype has not been described. Phylogenetic tree reconstruction showed that au2.g7373 is indeed the ortholog of Dm-senseless (see figure B.1). This could indicate that Tc-senseless fulfilled both functions in the last common ancestor. After a gene duplication the paralogs were used in different processes.

\subsubsection{Outlook RNAseq}

In summary the here described RNAseq after RNAi approach is robust and technically not too complex. It has great potential in the genetic deletion of certain tissues in embryos, which are not amenable to mechanical dissection because of their small size.

To check whether the ocular parasegment boundary has indeed a repressive function, RNAi against Wnt and hedghog pathway components followed by in situ stainings of anterior candidate genes (e.g. Tc-orthodenticle), could clarify this hypothesis.

A functional analysis of the paralogs of senseless in Drosophila and Tribolium would help understanding the ancestral role of the gene. 


\section{References}

A. Abzhanov and T. C. Kaufman. Homeotic genes and the arthropod head: Expression patterns of the labial, proboscipedia, and deformed genes in crustaceans and insects. Proceedings of the National Academy of Sciences of the United States of America, 96 (18):10224-10229, Aug. 1999. ISSN 0027-8424. URL http://www.ncbi.nlm.nih. gov/pmc/articles/PMC17870/. PMID: 10468590 PMCID: PMC17870. 2

S. F. Altschul, T. L. Madden, A. A. Schäffer, J. Zhang, Z. Zhang, W. Miller, and D. J. Lipman. Gapped BLAST and PSI-BLAST: a new generation of protein database search programs. Nucleic acids research, 25(17):3389-3402, Sept. 1997. ISSN 03051048. PMID: 9254694. 10, 17

S. Anders and W. Huber. Differential expression analysis for sequence count data. Genome biology, 11(10):R106, 2010. ISSN 1465-6914. doi: 10.1186/ gb-2010-11-10-r106. PMID: 20979621. 16

L. E. Anderson. Hoyer's solution as a rapid permanent mounting medium for bryophytes. The Bryologist, 57(3):242, Sept. 1954. ISSN 00072745. doi: 10. 2307/3240091. URL http://www.jstor.org/discover/10.2307/3240091?uid= 3737864\&uid=2129\&uid=2\&uid=70\&uid=4\&sid=21102875966867. 14

P. G. P. Andrews, Z. He, Y. R. Tzenov, C. Popadiuk, and K. R. Kao. Evidence of a novel role for pygopus in rRNA transcription. The Biochemical journal, 453(1): 61-70, July 2013. ISSN 1470-8728. doi: 10.1042/BJ20121667. PMID: 23517060. 59

P. Aza-Blanc, F. A. Ramrez-Weber, M. P. Laget, C. Schwartz, and T. B. Kornberg. Proteolysis that is inhibited by hedgehog targets cubitus interruptus protein to the nucleus and converts it to a repressor. Cell, 89(7):1043-1053, Jun 1997. 20, 53

D. A. Baker, B. Mille-Baker, S. M. Wainwright, D. Ish-Horowicz, and N. J. Dibb. Mae mediates MAP kinase phosphorylation of ets transcription factors in drosophila. 


\section{REFERENCES}

Nature, 411(6835):330-334, May 2001. ISSN 0028-0836. doi: 10.1038/35077122. URL http://www.nature.com/nature/journal/v411/n6835/full/411330a0.html. 35

A. Beermann, R. Prühs, R. Lutz, and R. Schröder. A context-dependent combination of wht receptors controls axis elongation and leg development in a short germ insect. Development (Cambridge, England), 138(13):2793-2805, July 2011. ISSN 1477-9129. doi: 10.1242/dev.063644. PMID: 21652652. 2, 15, 35

N. Berns, T. Kusch, R. Schröder, and R. Reuter. Expression, function and regulation of brachyenteron in the short germband insect tribolium castaneum. Development genes and evolution, 218(3-4):169-179, Apr. 2008. ISSN 0949-944X. doi: 10.1007/ s00427-008-0210-7. PMID: 18392878. 35

G. R. W. I. R. s. c. a. d. c. b. B. Bolker, L. Bonebakker, R. Gentleman, W. H. A. Liaw, T. Lumley, M. Maechler, A. Magnusson, S. Moeller, M. Schwartz, and B. Venables. gplots: Various $R$ programming tools for plotting data. 2013. URL http://CRAN. $\mathrm{R}$-project.org/package=gplots. R package version 2.11.0.1. 17

R. Bolognesi, L. Farzana, T. D. Fischer, and S. J. Brown. Multiple wnt genes are required for segmentation in the short-germ embryo of tribolium castaneum. Current biology: $C B, 18(20): 1624-1629$, Oct. 2008. ISSN 0960-9822. doi: 10.1016/j.cub.2008. 09.057. PMID: 18926702. 2, 5, 15, 52

R. Bolognesi, T. D. Fischer, and S. J. Brown. Loss of tc-arrow and canonical wnt signaling alters posterior morphology and pair-rule gene expression in the short-germ insect, tribolium castaneum. Development genes and evolution, 219(7):369-375, July 2009. ISSN 1432-041X. doi: 10.1007/s00427-009-0299-3. PMID: 19705150. 5, 15, 35

S. Brown, J. Fellers, T. Shippy, R. Denell, M. Stauber, and U. Schmidt-Ott. A strategy for mapping bicoid on the phylogenetic tree. Current biology: CB, 11(2):R43-44, Jan. 2001. ISSN 0960-9822. PMID: 11231138. 1

G. Bucher and M. Klingler. Divergent segmentation mechanism in the short germ insect tribolium revealed by giant expression and function. Development (Cambridge, England), 131(8):1729-1740, Apr. 2004. ISSN 0950-1991. doi: 10.1242/dev.01073. PMID: 15084458. 2

G. Bucher and M. Klingler. Tribolium mae expression suggests roles in terminal and midline patterning and in the specification of mesoderm. Development 


\section{REFERENCES}

genes and evolution, 215(9):478-481, Sept. 2005. ISSN 0949-944X. doi: 10.1007/ s00427-005-0004-0. PMID: 16025346. 35

G. Bucher and E. Wimmer. Beetle a-head. B.I.F. Futura, 20:164-169, 2005. 2, 4

G. Bucher, J. Scholten, and M. Klingler. Parental RNAi in tribolium (coleoptera). Current biology: CB, 12(3):R85-86, Feb. 2002. ISSN 0960-9822. PMID: 11839285. 15

J. D. Cande, V. S. Chopra, and M. Levine. Evolving enhancer-promoter interactions within the tinman complex of the flour beetle, tribolium castaneum. Development (Cambridge, England), 136(18):3153-3160, Sept. 2009. ISSN 1477-9129. doi: 10. 1242/dev.038034. PMID: 19700619. 35

S. Carbon, A. Ireland, C. J. Mungall, S. Shu, B. Marshall, and S. Lewis. AmiGO: online access to ontology and annotation data. Bioinformatics, 25(2):288-289, Jan. 2009. ISSN 1367-4803. doi: 10.1093/bioinformatics/btn615. URL http://www.ncbi.nlm . nih.gov/pmc/articles/PMC2639003/. PMID: 19033274 PMCID: PMC2639003. 88

A. C. Cerny, G. Bucher, R. Schröder, and M. Klingler. Breakdown of abdominal patterning in the tribolium kruppel mutant jaws. Development (Cambridge, England), 132(24):5353-5363, Dec. 2005. ISSN 0950-1991. doi: 10.1242/dev.02154. PMID: 16280347. 2

A. C. Cerny, D. Grossmann, G. Bucher, and M. Klingler. The tribolium ortholog of knirps and knirps-related is crucial for head segmentation but plays a minor role during abdominal patterning. Developmental biology, 321(1):284-294, Sept. 2008. ISSN 1095-564X. doi: 10.1016/j.ydbio.2008.05.527. PMID: 18586236. 3, 22, 52, 53

C. P. Choe and S. J. Brown. Evolutionary flexibility of pair-rule patterning revealed by functional analysis of secondary pair-rule genes, paired and sloppy-paired in the short-germ insect, tribolium castaneum. Developmental Biology, 302(1):281-294, Feb. 2007. ISSN 0012-1606. doi: 10.1016/j.ydbio.2006.09.037. URL http://www . sciencedirect.com/science/article/pii/S0012160606012395. 5, 36

C. P. Choe, S. C. Miller, and S. J. Brown. A pair-rule gene circuit defines segments sequentially in the short-germ insect tribolium castaneum. Proceedings of the National Academy of Sciences of the United States of America, 103(17):6560-6564, Apr. 2006. ISSN 0027-8424. doi: 10.1073/pnas.0510440103. PMID: 16611732. 2, 35, 54 


\section{REFERENCES}

S. Cohen and G. Jürgens. Drosophila headlines. Trends Genet, 7(8):267-272, Aug 1991. 2

S. M. Cohen and G. Jürgens. Mediation of drosophila head development by gap-like segmentation genes. Nature, 346(6283):482-485, Aug. 1990. ISSN 0028-0836. doi: 10.1038/346482a0. PMID: 1974035. 2, 58

T. Copf, R. Schröder, and M. Averof. Ancestral role of caudal genes in axis elongation and segmentation. Proceedings of the National Academy of Sciences of the United States of America, 101(51):17711-17715, Dec. 2004. ISSN 0027-8424. doi: 10.1073/ pnas.0407327102. PMID: 15598743. 35

F. Corpet. Multiple sequence alignment with hierarchical clustering. Nucleic acids research, 16(22):10881-10890, Nov. 1988. ISSN 0305-1048. PMID: 2849754. 95

M. Crozatier, D. Valle, L. Dubois, S. Ibnsouda, and A. Vincent. Collier, a novel regulator of drosophila head development, is expressed in a single mitotic domain. Curr Biol, 6(6):707-718, Jun 1996. 3

M. Crozatier, D. Valle, L. Dubois, S. Ibnsouda, and A. Vincent. Head versus trunk patterning in the drosophila embryo; collier requirement for formation of the intercalary segment. Development, 126(19):4385-4394, Oct 1999. 3

E. El-Sherif, M. Averof, and S. J. Brown. A segmentation clock operating in blastoderm and germband stages of tribolium development. Development (Cambridge, England), 139(23):4341-4346, Dec. 2012. ISSN 1477-9129. doi: 10.1242/dev.085126. PMID: 23095886. 2

L. Farzana and S. J. Brown. Hedgehog signaling pathway function conserved in tribolium segmentation. Development genes and evolution, 218(3-4):181-192, Apr. 2008. ISSN 0949-944X. doi: 10.1007/s00427-008-0207-2. PMID: 18392879. 2, 5, $15,36,52,59$

R. Finkelstein and N. Perrimon. The orthodenticle gene is regulated by bicoid and torso and specifies drosophila head development. Nature, 346(6283):485-488, Aug 1990. doi: 10.1038/346485a0. URL http://dx.doi.org/10.1038/346485a0. 2

J. Fu, N. Posnien, R. Bolognesi, T. D. Fischer, P. Rayl, G. Oberhofer, P. Kitzmann, S. J. Brown, and G. Bucher. Asymmetrically expressed axin required for anterior development in tribolium. Proceedings of the National Academy of Sciences of the 


\section{REFERENCES}

United States of America, 109(20):7782-7786, May 2012. ISSN 1091-6490. doi: 10.1073/pnas.1116641109. PMID: 22552230. 1, 15

A. Gallitano-Mendel and R. Finkelstein. Novel segment polarity gene interactions during embryonic head development in drosophila. Dev Biol, 192(2):599-613, Dec 1997. doi: 10.1006/dbio.1997.8753. URL http://dx.doi.org/10.1006/dbio.1997.8753. $3,28,56$

A. Gallitano-Mendel and R. Finkelstein. Ectopic orthodenticle expression alters segment polarity gene expression but not head segment identity in the drosophila embryo. Dev Biol, 199(1):125-137, Jul 1998. doi: 10.1006/dbio.1998.8917. URL http://dx.doi.org/10.1006/dbio.1998.8917. 3

W. M. Gelbart, M. A. Crosby, B. Matthews, W. P. Rindone, J. Chillemi, S. R. Twombly, D. Emmert, M. Ashburner, R. A. Drysdale, E. Whitfield, G. H. Millburn, A. de Grey, T. Kaufman, K. Matthews, D. Gilbert, V. B. Strelets, and C. Tolstoshev. FlyBase: a drosophila database. the FlyBase consortium. Nucleic Acids Research, 25(1):63-66, 1997. URL http://dblp.uni-trier.de/db/journals/nar/ nar25.html\#GelbartCMRCTEADWMGKMGST97. 17, 36

R. C. Gentleman, V. J. Carey, D. M. Bates, et al. Bioconductor: Open software development for computational biology and bioinformatics. Genome Biology, 5:R80, 2004. URL http://genomebiology.com/2004/5/10/R80. 16

A. J. Giraldez, R. R. Copley, and S. M. Cohen. HSPG modification by the secreted enzyme notum shapes the wingless morphogen gradient. Developmental cell, 2(5): 667-676, May 2002. ISSN 1534-5807. PMID: 12015973. 36

T. Girke. R \& bioconductor - manuals. URL http://manuals . bioinformatics . ucr . edu/home/R_BioCondManual. 16

D. Grossmann, J. Scholten, and N.-M. Prpic. Separable functions of wingless in distal and ventral patterning of the tribolium leg. Development genes and evolution, 219(910):469-479, Oct. 2009. ISSN 1432-041X. doi: 10.1007/s00427-009-0310-z. PMID: 20024581. 47

U. Grossniklaus, R. K. Pearson, and W. J. Gehring. The drosophila sloppy paired locus encodes two proteins involved in segmentation that show homology to mammalian transcription factors. Genes Dev, 6(6):1030-1051, Jun 1992. 2 


\section{REFERENCES}

M. Hahn and H. Jäckle. Drosophila goosecoid participates in neural development but not in body axis formation. The EMBO journal, 15(12):3077-3084, June 1996. ISSN 0261-4189. PMID: 8670808. 58

K. Handel, A. Basal, X. Fan, and S. Roth. Tribolium castaneum twist: gastrulation and mesoderm formation in a short-germ beetle. Development genes and evolution, 215(1):13-31, Jan. 2005. ISSN 0949-944X. doi: 10.1007/s00427-004-0446-9. PMID: 15645317. 59

A. M. Huang and G. M. Rubin. A misexpression screen identifies genes that can modulate RAS1 pathway signaling in drosophila melanogaster. Genetics, 156(3): 1219-1230, Nov. 2000. ISSN 0016-6731, 1943-2631. URL http://www.genetics . org/content/156/3/1219. PMID: 11063696. 35

H. Jafar-Nejad, A.-C. Tien, M. Acar, and H. J. Bellen. Senseless and daughterless confer neuronal identity to epithelial cells in the drosophila wing margin. Development, 133 (9):1683-1692, May 2006. ISSN 0950-1991, 1477-9129. doi: 10.1242/dev.02338. URL http://dev.biologists.org/content/133/9/1683. PMID: 16554363. 46

K. Jagla, T. Jagla, P. Heitzler, G. Dretzen, F. Bellard, and M. Bellard. ladybird, a tandem of homeobox genes that maintain late wingless expression in terminal and dorsal epidermis of the drosophila embryo. Development, 124(1):91-100, Jan. 1997. ISSN 0950-1991, 1477-9129. URL http://dev.biologists.org/content/124/1/ 91. PMID: 9006070. 35

G. Jekely and P. Friedrich. Characterization of two recombinant DrosophilaCalpains CALPA AND a NOVEL HOMOLOG, CALPB. Journal of Biological Chemistry, 274 (34):23893-23900, Aug. 1999. ISSN 0021-9258, 1083-351X. doi: 10.1074/jbc.274.34. 23893. URL http://www.jbc.org/content/274/34/23893. PMID: 10446155. 36

D. S. Johnston and C. Nüsslein-Volhard. The origin of pattern and polarity in the drosophila embryo. Cell, 68(2):201-219, Jan 1992. 1

M. Kanayama, Y. Akiyama-Oda, O. Nishimura, H. Tarui, K. Agata, and H. Oda. Travelling and splitting of a wave of hedgehog expression involved in spider-head segmentation. Nature Communications, 2:500, Oct. 2011. doi: 10.1038/ncomms1510. URL http://www. nature.com/ncomms/journal/v2/n10/full/ncomms1510.html. 3, 52, 54 


\section{REFERENCES}

D. Kent, E. W. Bush, and J. E. Hooper. Roadkill attenuates hedgehog responses through degradation of cubitus interruptus. Development (Cambridge, England), 133(10):2001-2010, May 2006. ISSN 0950-1991. doi: 10.1242/dev.02370. PMID: 16651542. 36

S. Kittelmann, J. Ulrich, N. Posnien, and G. Bucher. Changes in anterior head patterning underlie the evolution of long germ embryogenesis. Developmental biology, 374 (1):174-184, Feb. 2013. ISSN 1095-564X. doi: 10.1016/j.ydbio.2012.11.026. PMID: 23201022. 2

K. Kotkamp, M. Klingler, and M. Schoppmeier. Apparent role of tribolium orthodenticle in anteroposterior blastoderm patterning largely reflects novel functions in dorsoventral axis formation and cell survival. Development (Cambridge, England), 137(11):1853-1862, June 2010. ISSN 1477-9129. doi: 10.1242/dev.047043. PMID: 20431120. 3, 15

T. Kramps, O. Peter, E. Brunner, D. Nellen, B. Froesch, S. Chatterjee, M. Murone, S. Züllig, and K. Basler. Wnt/wingless signaling requires BCL9/legless-mediated recruitment of pygopus to the nuclear beta-catenin-TCF complex. Cell, 109(1):4760, Apr. 2002. ISSN 0092-8674. PMID: 11955446. 59

B. Langmead and S. L. Salzberg. Fast gapped-read alignment with bowtie 2. Nature Methods, 9(4):357-359, Apr. 2012. ISSN 1548-7091. doi: 10.1038/nmeth.1923. URL http://www .nature.com/nmeth/journal/v9/n4/full/nmeth.1923.html. 16

G. Lauter, I. Söll, and G. Hauptmann. Multicolor fluorescent in situ hybridization to define abutting and overlapping gene expression in the embryonic zebrafish brain. Neural Development, 6(1):10, Apr. 2011. ISSN 1749-8104. doi: 10.1186/1749-8104-6-10. URL http://www. neuraldevelopment.com/content/6/1/10/abstract. PMID: 21466670. 11

J. A. Lengyel and D. D. Iwaki. It takes guts: the drosophila hindgut as a model system for organogenesis. Developmental biology, 243(1):1-19, Mar. 2002. ISSN 0012-1606. doi: 10.1006/dbio.2002.0577. PMID: 11846473. 60

H. Li, B. Handsaker, A. Wysoker, T. Fennell, J. Ruan, N. Homer, G. Marth, G. Abecasis, R. Durbin, and 1000 Genome Project Data Processing Subgroup. The sequence Alignment/Map format and SAMtools. Bioinformatics (Oxford, England), 25(16): 2078-2079, Aug. 2009. ISSN 1367-4811. doi: 10.1093/bioinformatics/btp352. PMID: 19505943. 16 


\section{REFERENCES}

P. Z. Liu and T. C. Kaufman. Short and long germ segmentation: unanswered questions in the evolution of a developmental mode. Evolution $\&$ development, 7(6):629646, Dec. 2005. ISSN 1520-541X. doi: 10.1111/j.1525-142X.2005.05066.x. PMID: 16336416. 1

C. M. Luque and M. Miln. Growth control in the proliferative region of the drosophila eyehead primordium: The elbownoc gene complex. Developmental Biology, 301(2): 327-339, Jan. 2007. ISSN 0012-1606. doi: 10.1016/j.ydbio.2006.07.050. URL http: //www.sciencedirect.com/science/article/pii/S0012160606011559. 35

B. L. Martin and D. Kimelman. Wnt signaling and the evolution of embryonic posterior development. Current Biology, 19(5):R215-R219, Mar. 2009. ISSN 0960-9822. doi: 10.1016/j.cub.2009.01.052. URL http://www.sciencedirect.com/science/ article/pii/S0960982209006241. 59

W. McGinnis and R. Krumlauf. Homeobox genes and axial patterning. Cell, 68(2): 283-302, Jan. 1992. ISSN 0092-8674. PMID: 1346368. 1

J. Mohler. Spatial regulation of segment polarity gene expression in the anterior terminal region of the drosophila blastoderm embryo. Mech Dev, 50(2-3):151-161, Apr 1995. 3

D. Morisato and K. V. Anderson. The spätzle gene encodes a component of the extracellular signaling pathway establishing the dorsal-ventral pattern of the drosophila embryo. Cell, 76(4):677-688, Feb. 1994. ISSN 0092-8674. PMID: 8124709. 36

A. Mortazavi, B. A. Williams, K. McCue, L. Schaeffer, and B. Wold. Mapping and quantifying mammalian transcriptomes by RNA-Seq. Nature methods, 5(7):621-628, July 2008. ISSN 1548-7105. doi: 10.1038/nmeth.1226. PMID: 18516045. 6

P. Müller, D. Kuttenkeuler, V. Gesellchen, M. P. Zeidler, and M. Boutros. Identification of JAK/STAT signalling components by genome-wide RNA interference. Nature, 436 (7052):871-875, Aug. 2005. ISSN 0028-0836. doi: 10.1038/nature03869. URL http: //www.nature.com/nature/journal/v436/n7052/full/nature03869.html. 36

U. Nagalakshmi, Z. Wang, K. Waern, C. Shou, D. Raha, M. Gerstein, and M. Snyder. The transcriptional landscape of the yeast genome defined by RNA sequencing. Science (New York, N.Y.), 320(5881):1344-1349, June 2008. ISSN 1095-9203. doi: 10.1126/science.1158441. PMID: 18451266. 6 


\section{REFERENCES}

E. Neuwirth. RColorBrewer: ColorBrewer palettes. 2011. URL http://CRAN. $\mathrm{R}$-project.org/package=RColorBrewer. $\mathrm{R}$ package version 1.0-5. 17

R. Nolo, L. A. Abbott, and H. J. Bellen. Senseless, a zn finger transcription factor, is necessary and sufficient for sensory organ development in drosophila. Cell, 102(3): 349-362, Aug. 2000. ISSN 0092-8674. PMID: 10975525. 46, 47, 60

E. Ntini and E. A. Wimmer. Second order regulator collier directly controls intercalaryspecific segment polarity gene expression. Developmental biology, 360(2):403-414, Dec. 2011a. ISSN 1095-564X. doi: 10.1016/j.ydbio.2011.09.035. PMID: 22005665. 3

E. Ntini and E. A. Wimmer. Unique establishment of procephalic head segments is supported by the identification of cis-regulatory elements driving segment-specific segment polarity gene expression in drosophila. Development genes and evolution, 221(1):1-16, May 2011b. ISSN 1432-041X. doi: 10.1007/s00427-011-0354-8. PMID: 21399984. 3

C. Nüsslein-Volhard and E. Wieschaus. Mutations affecting segment number and polarity in drosophila. Nature, 287(5785):795-801, Oct. 1980. ISSN 0028-0836. PMID: 6776413. 1

D. I. Oppenheimer, A. M. MacNicol, and N. H. Patel. Functional conservation of the wingless-engrailed interaction as shown by a widely applicable baculovirus misexpression system. Current biology: CB, 9(22):1288-1296, Nov. 1999. ISSN 0960-9822. PMID: 10574758. 6, 56

K. A. Panfilio, G. Oberhofer, and S. Roth. High plasticity in epithelial morphogenesis during insect dorsal closure. Biology open, 2(11):1108-1118, 2013. ISSN 2046-6390. doi: 10.1242/bio.20136072. PMID: 24244847. 14

M. J. Pankratz and H. Jäckle. Making stripes in the drosophila embryo. Trends in genetics: TIG, 6(9):287-292, Sept. 1990. ISSN 0168-9525. PMID: 2238086. 2

M. Pechmann, A. P. McGregor, E. E. Schwager, N. M. Feitosa, and W. G. M. Damen. Dynamic gene expression is required for anterior regionalization in a spider. Proceedings of the National Academy of Sciences of the United States of America, 106 (5):1468-1472, Feb. 2009. ISSN 1091-6490. doi: 10.1073/pnas.0811150106. PMID: 19147844. 3,54 


\section{REFERENCES}

A. D. Peel, J. Schanda, D. Grossmann, F. Ruge, G. Oberhofer, A. F. Gilles, J. B. Schinko, M. Klingler, and G. Bucher. Tc-knirps plays different roles in the specification of antennal and mandibular parasegment boundaries and is regulated by a pair-rule gene in the beetle tribolium castaneum. BMC developmental biology, 13 : 25, 2013. ISSN 1471-213X. doi: 10.1186/1471-213X-13-25. PMID: 23777260. 3, 22, 52,54

C. P. Petersen and P. W. Reddien. Wnt signaling and the polarity of the primary body axis. Cell, 139(6):1056-1068, Dec. 2009. ISSN 1097-4172. doi: 10.1016/j.cell.2009. 11.035. PMID: 20005801. 59

N. Posnien and G. Bucher. Formation of the insect head involves lateral contribution of the intercalary segment, which depends on tc-labial function. Developmental biology, 338(1):107-116, Feb. 2010. ISSN 1095-564X. doi: 10.1016/j.ydbio.2009.11.010. PMID: 19913530. 2

N. Posnien, J. B. Schinko, S. Kittelmann, and G. Bucher. Genetics, development and composition of the insect head-a beetle's view. Arthropod structure $\&$ development, 39(6):399-410, Nov. 2010. ISSN 1873-5495. doi: 10.1016/j.asd.2010.08.002. PMID: 20800703. 5

N. Posnien, N. D. B. Koniszewski, H. J. Hein, and G. Bucher. Candidate gene screen in the red flour beetle tribolium reveals six3 as ancient regulator of anterior median head and central complex development. PLoS genetics, 7(12):e1002416, Dec. 2011. ISSN 1553-7404. doi: 10.1371/journal.pgen.1002416. PMID: 22216011. 2, 5, 36, 41, 58

T. Sandmann, J. S. Jakobsen, and E. E. M. Furlong. ChIP-on-chip protocol for genomewide analysis of transcription factor binding in drosophila melanogaster embryos. Nature protocols, 1(6):2839-2855, 2006. ISSN 1750-2799. doi: 10.1038/nprot.2006. 383. PMID: 17406543. 10

A. F. Sarrazin, A. D. Peel, and M. Averof. A segmentation clock with two-segment periodicity in insects. Science (New York, N.Y.), 336(6079):338-341, Apr. 2012. ISSN 1095-9203. doi: 10.1126/science.1218256. PMID: 22403177. 2

J. Schinko, N. Posnien, S. Kittelmann, N. Koniszewski, and G. Bucher. Single and double whole-mount in situ hybridization in red flour beetle (tribolium) embryos. Cold Spring Harbor protocols, 2009(8):pdb.prot5258, Aug. 2009. ISSN 1559-6095. doi: 10.1101/pdb.prot5258. PMID: 20147234. 10, 12 


\section{REFERENCES}

J. B. Schinko, N. Kreuzer, N. Offen, N. Posnien, E. A. Wimmer, and G. Bucher. Divergent functions of orthodenticle, empty spiracles and buttonhead in early head patterning of the beetle tribolium castaneum (coleoptera). Developmental biology, 317 (2):600-613, May 2008. ISSN 1095-564X. doi: 10.1016/j.ydbio.2008.03.005. PMID: 18407258. $3,24,25,30,52,58$

J. B. Schinko, K. Hillebrand, and G. Bucher. Heat shock-mediated misexpression of genes in the beetle tribolium castaneum. Development Genes and Evolution, 222(5): 287-298, Sept. 2012. ISSN 0949-944X, 1432-041X. doi: 10.1007/s00427-012-0412-x. URL http://link.springer.com/article/10.1007/s00427-012-0412-x. 9, 15, 25

M. Schoppmeier and R. Schroeder. Maternal torso signaling controls body axis elongation in a short germ insect. Current biology: CB, 15(23):2131-2136, Dec. 2005. ISSN 0960-9822. doi: 10.1016/j.cub.2005.10.036. PMID: 16332539. 2, 15, 30

M. Schoppmeier, S. Fischer, C. Schmitt-Engel, U. Löhr, and M. Klingler. An ancient anterior patterning system promotes caudal repression and head formation in ecdysozoa. Current biology: CB, 19(21):1811-1815, Nov. 2009. ISSN 1879-0445. doi: 10.1016/j.cub.2009.09.026. PMID: 19818622. 1

R. Schröder. The genes orthodenticle and hunchback substitute for bicoid in the beetle tribolium. Nature, 422(6932):621-625, Apr. 2003. ISSN 0028-0836. doi: 10.1038/ nature01536. PMID: 12687002. 3

R. Schroder, C. Eckert, C. Wolff, and D. Tautz. Conserved and divergent aspects of terminal patterning in the beetle tribolium castaneum. Proceedings of the National Academy of Sciences of the United States of America, 97(12):6591-6596, June 2000. ISSN 0027-8424. doi: 10.1073/pnas.100005497. PMID: 10823887. 58

C. Schulz, R. Schröder, B. Hausdorf, C. Wolff, and D. Tautz. A caudal homologue in the short germ band beetle tribolium shows similarities to both, the drosophila and the vertebrate caudal expression patterns. Development genes and evolution, 208(5): 283-289, July 1998. ISSN 0949-944X. PMID: 9683744. 35

R. Sharma, A. Beermann, and R. Schröder. FGF signalling controls anterior extraembryonic and embryonic fate in the beetle tribolium. Developmental biology, 381(1): 121-133, Sept. 2013. ISSN 1095-564X. doi: 10.1016/j.ydbio.2013.05.031. PMID: 23769707. 2 


\section{REFERENCES}

J. B. Singer, R. Harbecke, T. Kusch, R. Reuter, and J. A. Lengyel. Drosophila brachyenteron regulates gene activity and morphogenesis in the gut. Development (Cambridge, England), 122(12):3707-3718, Dec. 1996. ISSN 0950-1991. PMID: 9012492. 35

A. Sokoloff. The biology of Tribolium: with special emphasis on genetic aspects. Clarendon Press, 1974. 9

R. J. Sommer and D. Tautz. Involvement of an orthologue of the drosophila pairrule gene hairy in segment formation of the short germ-band embryo of tribolium (coleoptera). Nature, 361(6411):448-450, Feb. 1993. ISSN 0028-0836. doi: 10.1038/ 361448a0. PMID: 8429884. 35

R. J. Sommer and D. Tautz. Expression patterns of twist and snail in tribolium (coleoptera) suggest a homologous formation of mesoderm in long and short germ band insects. Developmental genetics, 15(1):32-37, 1994. ISSN 0192-253X. doi: 10.1002/dvg.1020150105. PMID: 8187348. 35

J. E. Stajich, D. Block, K. Boulez, S. E. Brenner, S. A. Chervitz, C. Dagdigian, G. Fuellen, J. G. R. Gilbert, I. Korf, H. Lapp, H. Lehväslaiho, C. Matsalla, C. J. Mungall, B. I. Osborne, M. R. Pocock, P. Schattner, M. Senger, L. D. Stein, E. Stupka, M. D. Wilkinson, and E. Birney. The bioperl toolkit: Perl modules for the life sciences. Genome research, 12(10):1611-1618, Oct. 2002. ISSN 1088-9051. doi: 10.1101/gr.361602. PMID: 12368254. 10, 17

M. Stanke and S. Waack. Gene prediction with a hidden markov model and a new intron submodel. Bioinformatics, 19(suppl 2):ii215-ii225, Sept. 2003. ISSN 1367-4803, 1460-2059. doi: 10.1093/bioinformatics/btg1080. URL http://bioinformatics . oxfordjournals.org/content/19/suppl_2/ii215. PMID: 14534192. 10

M. Stauber, H. Jäckle, and U. Schmidt-Ott. The anterior determinant bicoid of drosophila is a derived hox class 3 gene. Proceedings of the National Academy of Sciences of the United States of America, 96(7):3786-3789, Mar. 1999. ISSN 00278424. PMID: 10097115. 1

M. Sultan, M. H. Schulz, H. Richard, A. Magen, A. Klingenhoff, M. Scherf, M. Seifert, T. Borodina, A. Soldatov, D. Parkhomchuk, D. Schmidt, S. O'Keeffe, S. Haas, M. Vingron, H. Lehrach, and M.-L. Yaspo. A global view of gene activity and alternative splicing by deep sequencing of the human transcriptome. Science (New York, N.Y.), 321(5891):956-960, Aug. 2008. ISSN 1095-9203. doi: 10.1126/science.1160342. PMID: 18599741. 6 


\section{REFERENCES}

N. Snchez-Soriano and S. Russell. Regulatory mutations of the drosophila sox gene dichaete reveal new functions in embryonic brain and hindgut development. Developmental Biology, 220(2):307-321, Apr. 2000. ISSN 0012-1606. doi: 10. 1006/dbio.2000.9648. URL http://www.sciencedirect.com/science/article/ pii/S0012160600996489. 35

D. Tautz, M. Friedrich, and R. Schröder. Insect embryogenesis what is ancestral and what is derived? Development, 1994(Supplement):193-199, Jan. 1994. ISSN 09501991, 1477-9129. URL http://dev.biologists.org/content/1994/Supplement/ 193. 1

R. C. Team. R: A Language and Environment for Statistical Computing. Vienna, Austria, 2013. URL http://www.R-project.org/. 16

Tribolium Genome Sequencing Consortium, S. Richards, R. A. Gibbs, G. M. Weinstock, S. J. Brown, R. Denell, R. W. Beeman, R. Gibbs, R. W. Beeman, S. J. Brown, G. Bucher, M. Friedrich, C. J. P. Grimmelikhuijzen, M. Klingler, M. Lorenzen, S. Richards, S. Roth, R. Schröder, D. Tautz, E. M. Zdobnov, D. Muzny, R. A. Gibbs, G. M. Weinstock, T. Attaway, S. Bell, C. J. Buhay, M. N. Chandrabose, D. Chavez, K. P. Clerk-Blankenburg, A. Cree, M. Dao, C. Davis, J. Chacko, H. Dinh, S. Dugan-Rocha, G. Fowler, T. T. Garner, J. Garnes, A. Gnirke, A. Hawes, J. Hernandez, S. Hines, M. Holder, J. Hume, S. N. Jhangiani, V. Joshi, Z. M. Khan, L. Jackson, C. Kovar, A. Kowis, S. Lee, L. R. Lewis, J. Margolis, M. Morgan, L. V. Nazareth, N. Nguyen, G. Okwuonu, D. Parker, S. Richards, S.-J. Ruiz, J. Santibanez, J. Savard, S. E. Scherer, B. Schneider, E. Sodergren, D. Tautz, S. Vattahil, D. Villasana, C. S. White, R. Wright, Y. Park, R. W. Beeman, J. Lord, B. Oppert, M. Lorenzen, S. Brown, L. Wang, J. Savard, D. Tautz, S. Richards, G. Weinstock, R. A. Gibbs, Y. Liu, K. Worley, G. Weinstock, C. G. Elsik, J. T. Reese, E. Elhaik, G. Landan, D. Graur, P. Arensburger, P. Atkinson, R. W. Beeman, J. Beidler, S. J. Brown, J. P. Demuth, D. W. Drury, Y.-Z. Du, H. Fujiwara, M. Lorenzen, V. Maselli, M. Osanai, Y. Park, H. M. Robertson, Z. Tu, J.-j. Wang, S. Wang, S. Richards, H. Song, L. Zhang, E. Sodergren, D. Werner, M. Stanke, B. Morgenstern, V. Solovyev, P. Kosarev, G. Brown, H.-C. Chen, O. Ermolaeva, W. Hlavina, Y. Kapustin, B. Kiryutin, P. Kitts, D. Maglott, K. Pruitt, V. Sapojnikov, A. Souvorov, A. J. Mackey, R. M. Waterhouse, S. Wyder, E. M. Zdobnov, E. M. Zdobnov, S. Wyder, E. V. Kriventseva, T. Kadowaki, P. Bork, M. Aranda, R. Bao, A. Beermann, N. Berns, R. Bolognesi, F. Bonneton, D. Bopp, S. J. Brown, G. Bucher, T. Butts, A. Chaumot, R. E. Denell, D. E. K. Ferrier, M. Friedrich, C. M. Gordon, 


\section{REFERENCES}

M. Jindra, M. Klingler, Q. Lan, H. M. G. Lattorff, V. Laudet, C. von Levetsow, Z. Liu, R. Lutz, J. A. Lynch, R. N. da Fonseca, N. Posnien, R. Reuter, S. Roth, J. Savard, J. B. Schinko, C. Schmitt, M. Schoppmeier, R. Schröder, T. D. Shippy, F. Simonnet, H. Marques-Souza, D. Tautz, Y. Tomoyasu, J. Trauner, M. Van der Zee, M. Vervoort, N. Wittkopp, E. A. Wimmer, X. Yang, A. K. Jones, D. B. Sattelle, P. R. Ebert, D. Nelson, J. G. Scott, R. W. Beeman, S. Muthukrishnan, K. J. Kramer, Y. Arakane, R. W. Beeman, Q. Zhu, D. Hogenkamp, R. Dixit, B. Oppert, H. Jiang, Z. Zou, J. Marshall, E. Elpidina, K. Vinokurov, C. Oppert, Z. Zou, J. Evans, Z. Lu, P. Zhao, N. Sumathipala, B. Altincicek, A. Vilcinskas, M. Williams, D. Hultmark, C. Hetru, H. Jiang, C. J. P. Grimmelikhuijzen, F. Hauser, G. Cazzamali, M. Williamson, Y. Park, B. Li, Y. Tanaka, R. Predel, S. Neupert, J. Schachtner, P. Verleyen, F. Raible, P. Bork, M. Friedrich, K. K. O. Walden, H. M. Robertson, S. Angeli, S. Fort, G. Bucher, S. Schuetz, R. Maleszka, E. A. Wimmer, R. W. Beeman, M. Lorenzen, Y. Tomoyasu, S. C. Miller, D. Grossmann, and G. Bucher. The genome of the model beetle and pest tribolium castaneum. Nature, 452(7190):949955, Apr. 2008. ISSN 1476-4687. doi: 10.1038/nature06784. PMID: 18362917. 10

R. I. Tuxworth, V. Vivancos, M. B. O'Hare, and G. Tear. Interactions between the juvenile batten disease gene, CLN3, and the notch and JNK signalling pathways. Human molecular genetics, 18(4):667-678, Feb. 2009. ISSN 1460-2083. doi: 10. 1093/hmg/ddn396. PMID: 19028667. 36

A. Untergasser, I. Cutcutache, T. Koressaar, J. Ye, B. C. Faircloth, M. Remm, and S. G. Rozen. Primer3new capabilities and interfaces. Nucleic Acids Research, page gks596, June 2012. ISSN 0305-1048, 1362-4962. doi: 10.1093/nar/gks596. URL http://nar . oxfordjournals.org/content/early/2012/06/21/nar.gks596. PMID: 22730293. 9

R. Urbach. A procephalic territory in drosophila exhibiting similarities and dissimilarities compared to the vertebrate midbrain/hindbrain boundary region. Neural development, 2:23, 2007. ISSN 1749-8104. doi: 10.1186/1749-8104-2-23. PMID: 17983473. 5

Q. T. Wang and R. A. Holmgren. Nuclear import of cubitus interruptus is regulated by hedgehog via a mechanism distinct from ci stabilization and ci activation. Development (Cambridge, England), 127(14):3131-3139, July 2000. ISSN 0950-1991. PMID: 10862750. 35 
D. Weigel, G. Jürgens, M. Klingler, and H. Jäckle. Two gap genes mediate maternal terminal pattern information in drosophila. Science (New York, N.Y.), 248(4954): 495-498, Apr. 1990. ISSN 0036-8075. PMID: 2158673. 58

H. Wickham. ggplot2: elegant graphics for data analysis. Springer New York, 2009. ISBN 978-0-387-98140-6. URL http://had.co.nz/ggplot2/book. 17

B. T. Wilhelm, S. Marguerat, S. Watt, F. Schubert, V. Wood, I. Goodhead, C. J. Penkett, J. Rogers, and J. Bähler. Dynamic repertoire of a eukaryotic transcriptome surveyed at single-nucleotide resolution. Nature, 453(7199):1239-1243, June 2008. ISSN 1476-4687. doi: 10.1038/nature07002. PMID: 18488015. 6

E. A. Wimmer, S. M. Cohen, H. Jäckle, and C. Desplan. buttonhead does not contribute to a combinatorial code proposed for drosophila head development. Development, 124(8):1509-1517, Apr 1997. 3

W. Wurst and L. Bally-Cuif. Neural plate patterning: upstream and downstream of the isthmic organizer. Nature reviews. Neuroscience, 2(2):99-108, Feb. 2001. ISSN 1471-003X. doi: 10.1038/35053516. PMID: 11253000. 5

X. Yang, M. Weber, N. Zarinkamar, N. Posnien, F. Friedrich, B. Wigand, R. Beutel, W. G. M. Damen, G. Bucher, M. Klingler, and M. Friedrich. Probing the drosophila retinal determination gene network in tribolium (II): the pax6 genes eyeless and twin of eyeless. Developmental biology, 333(1):215-227, Sept. 2009. ISSN 1095-564X. doi: 10.1016/j.ydbio.2009.06.013. PMID: 19527703. 36, 58 
REFERENCES 


\section{Appendix A}

\section{Clones and Primer}

Table A.1: Primer to clone genes and for dsRNA templates

\begin{tabular}{|c|c|c|}
\hline Gene & Primer & Sequence \\
\hline \multirow[t]{4}{*}{ Tc-frizzled 1} & frz1up1 & ATGAAGCCGCTGCTCCTGCTG \\
\hline & frz1lo1 & TTAAACGTAAGCCGCATGTCTG \\
\hline & frz1up2 & CTCTGCGTGGAGAAACACAAC \\
\hline & frz1lo2 & GCGGACAAGGCACGGCATAG \\
\hline \multirow[t]{4}{*}{ Tc-frizzled2 } & frz2up1 & ATGATGGGGTACACGAGGTTATC \\
\hline & frz2lo1 & TCATACGTGACTCAACGGGGGC \\
\hline & frz2up2 & AGCGTTGCGAGGACATCACCATC \\
\hline & frz2lo2 & AGCATCCACTCGTTATGGAATGC \\
\hline \multirow[t]{6}{*}{ Tc-patched } & Ptcup1 & AGTCGGACCTGTACACCAGG \\
\hline & Ptclo1 & CTAGCTACTGGGGCACGGTTC \\
\hline & Ptcup2T7 & TAATACGACTCACTATAGGCAGGCGAATCTAACACCGAA \\
\hline & Ptclo2T7 & TAATACGACTCACTATAGGGCCAACGATCAAGTCTTCCG \\
\hline & ptcup2T7 & TAATACGACTCACTATAGGACTTGTTGACTGCGGAGGTGATC \\
\hline & ptclo2T7 & TAATACGACTCACTATAGGGCACGGCACGTCAGTCTTTCG \\
\hline \multirow{6}{*}{$\begin{array}{l}\text { Tc- } \\
\text { smoothened }\end{array}$} & Smoup1 & ATGTACCTCTTGTTTGTGCTAAT \\
\hline & Smolo1 & TCATAACAACTCTCTCAGTTCTGG \\
\hline & Smoup2T7 & TAATACGACTCACTATAGGGATCGTCTTCTGCATCATCAC \\
\hline & Smolo2T7 & TAATACGACTCACTATAGGGGACGCAGCTCATGTGCCA \\
\hline & smoup2T7 & TAATACGACTCACTATAGGGCGCGAAGACATCGTTTGCA \\
\hline & smolo2T7 & TAATACGACTCACTATAGGGGATGAACCAACCGCTGTACG \\
\hline \multirow[t]{6}{*}{ Tc-wntless } & wntlF1 & ATGCCGGGAACAATCCTCGA \\
\hline & wntlR1 & TTACTCAATACTAGCTTTTGAAGC \\
\hline & wntlF2 & CATGAACTACTGGGTCACTCG \\
\hline & wntlR2 & CCGGTAGATTATACCTTCGTAG \\
\hline & wntllo2T7 & TAATACGACTCACTATAGGGTGCGTGACAGCAAATGCAC \\
\hline & wntlup2T7 & TAATACGACTCACTATAGGTGCTCTCTTTCTGGCTCGTG \\
\hline
\end{tabular}




\section{A. CLONES AND PRIMER}

Table A.2: Primer for clones obtained from colleagues

\begin{tabular}{|c|c|c|c|}
\hline Gene & cloned by & Primer & Sequence \\
\hline \multirow[t]{3}{*}{ Tc-hedgehog } & Evgenia Ntini & Hhup1T7 & TAATACGACTCACTATAGGGAGATGTAAGGAGAAGTTGAAC \\
\hline & & Hhlo2T7 & TAATACGACTCACTATAGGCTGAAGCCAGGGTTATGTGG \\
\hline & & Hhup2T7 & TAATACGACTCACTATAGGCAGTGACCCCATCTCATTTG \\
\hline \multirow[t]{4}{*}{ Tc-axin } & Nico Posnien & Axlo2T7 & TAATACGACTCACTATAGGTCGGTCCTCGCATCTGAATG \\
\hline & & Axup2T7 & TAATACGACTCACTATAGGGAGAGCAATGTTGACGGCAG \\
\hline & & Axilo1T7 & TAATACGACTCACTATAGGCCTGGTCTGCCGTCAACATT \\
\hline & & Axiup1T7 & TAATACGACTCACTATAGGCGTTTCATCCCACGAACCCA \\
\hline \multirow[t]{2}{*}{ Tc-arrow } & Nico Posnien & Arrlo1T7 & TAATACGACTCACTATAGGCCTTGAACCACGACTAAAACATG \\
\hline & & Arrup1T7 & TAATACGACTCACTATAGGTGCCATCACGAAAGAAGCCGA \\
\hline
\end{tabular}

Table A.3: Primer for the RNAseq in situ screen candidates

\begin{tabular}{|c|c|c|c|}
\hline $\mathrm{Nr}$ & au2ID & FWD & REV \\
\hline 1 & 216 & GCAGGTGGTCTGAACTTCTGAGGTA & GCCGGGTCTTGCTTAATATGTCAGT \\
\hline 2 & 219 & GTCCGTTCTCTCATCATCAGTCAC & ATAATAGTTGCTGCCAAGTGCTACG \\
\hline 3 & 1265 & CGAAATGACCATTTACGGCAAGTTA & CTTGGTTAGCGTAGGAGTCCCAGTT \\
\hline 4 & 1272 & CGAATACATCACATTAAGGCTGACA & TCCAACTGAATATCTTCCAACTGTG \\
\hline 5 & 1604 & CTTGCTGCTACAACCACTACGAAT & GTCTACATGGACCTTGGTTGGTTTA \\
\hline 6 & 1826 & CACCTACATCAGAAATCCCAACTCG & TTTAAACAAATGACTCACCCCGTTG \\
\hline 7 & 1922 & ATCTATCATCGAGGCCGTAACTGT & ATCTACATGGACCTTGGTTGGTTTA \\
\hline 8 & 2437 & AACTATGTGCCACAAGCAAATGAC & TAAGTCCTCTTCAAATCATCCTTGC \\
\hline 9 & 2544 & GGGCCTGAATCCTTATCTCAATTAT & TAATATCACACAGTCACGTCCACAC \\
\hline 10 & 2727 & GAAACTATCCGACTGCTTGTGATTT & TTACTCTTGCСТTTAACCAAAGTCG \\
\hline 11 & 2852 & GAGCAAGACCACACATCAGTGATAC & GCTTTTGATCAGCAGTTAGGGATAA \\
\hline 12 & 3254 & GGAGGTGTAATAATCTCGCAATCC & GCTTGGGAGTAAGCGATGTAGAAA \\
\hline 13 & 3393 & ATCCCTGTATTACGAATCCCTTTGT & ATCATAGACCACAGTTGGAACGAAT \\
\hline 15 & 3432 & TTTAACAGCACCTGGTGAACACTAC & AATCACATCACGTGCTATGGAACTA \\
\hline 16 & 4667 & CTCCAGAAGAAGGGTGTGTAAATGT & ATTTGCCAAGAAGTAAACAGTCTCG \\
\hline 18 & 5115 & CCCCTACGAATGCATAGAAATATGA & TATTACACTTCCGCGTTATTACCTG \\
\hline 19 & 5536 & AGGGGTAGCTAGTTATCGTCTTCGT & CGGGTTTTCATCGCTATACACTTAT \\
\hline 20 & 5804 & AGAAGAAGAAGAAAGAGCGGAAAGT & AACACTGGTTACATAAACTCGTCCA \\
\hline 21 & 5809 & TCATCAGTTACGAGAGCAAAGAACA & CGAATTGTAAATACACTGAGCGTTG \\
\hline 22 & 6283 & CACATTTCTGATTATGCACAGCAC & TGGCAAGTAGACTAGCACGTATGAG \\
\hline 23 & 6578 & CGATAATTCACTATGAGGGAAATCG & CTTTGATCCTTGAATCGTTCAGTTC \\
\hline 24 & 6598 & AGTACGATTACGTGGAGGACAAATC & AACCACCCCTTTGTTATTATCAGGT \\
\hline 25 & 6823 & AACATACGTCGGTCAAGCCTACATC & GCGCTCATCGTCCTTGTCATTATAG \\
\hline
\end{tabular}


Table A.3 - continued from previous page

\begin{tabular}{|c|c|c|c|}
\hline $\mathbf{N r}$ & au2ID & FWD & REV \\
\hline 26 & 7373 & ССТTСТСТСТАGTCTACCCCAAGGA & AGTCACTCTCATGGCACTAACTGCT \\
\hline 28 & 7815 & TTCGCCCAAGTTCATGTGTAT & GTCCACCCGTTGAGTGAACTA \\
\hline 29 & 7984 & TCCGGTGAATATTACAACAGAAACC & TCAGTATCAATGGTCATTCCGTCTA \\
\hline 30 & 8329 & TTTCGTCTTCACCCTTTTTGTCGTTAC & GTGATGCTCCCCTTGTCCTGGTTTAT \\
\hline 31 & 8382 & GCAGGCAACGAAACACTTTAC & TAAATGGCACTGGCTGCTATC \\
\hline 32 & 8413 & TTTATTTGAGCGTAGACCCGTACAT & CTTCCССТCTTTGATCCACTTTTTA \\
\hline 33 & 8500 & ACTTTCTGCCTCTTCACAGTTCAGT & GACTCGCAACTTTACCAACAGAATC \\
\hline 34 & 8732 & GAAAATCAACACGCCATTACG & CAATCGGTACCACGACTCAAG \\
\hline 35 & 9385 & CACGACAAATACGCAGTTATCAGAA & ATATGTCCACAGCCAAATTTCCATC \\
\hline 36 & 10180 & AACTAGTCGATGGGTTCCTCTACCT & GCACAACCTTTCCACAACTTTACTT \\
\hline 37 & 10490 & CTCTGGAATACAACACTCCAGACAA & GCATCTTAGTCTTGTCACCAATTCC \\
\hline 38 & 10495 & ACGTACTTGCTATGTGTCCAAAGTG & ATAGACATGATAATGAGGCGATGGT \\
\hline 39 & 10496 & CTTACTCGACTTCTTCGTCAAGTCC & GGGTAGTTATTGCTTGATCGTTCTG \\
\hline 40 & 10497 & CAATTACGACTACTCGGACTTCACC & GACTGTCTCACCTCGTCTTCTGAC \\
\hline 41 & 11365 & ACTACGTTTCCGCTCGTTTGTATTA & CCTTGAATTTGGTTAAAGAGGAGGT \\
\hline 43 & 12008 & GATTATTTTCTCCAAAATGACGCTTCC & GTGTGAAGTCCAGACCACTCAATTTCT \\
\hline 44 & 12107 & GTGTCAGAAGTGAACGAGTTGAAGA & TCACTTTCTCGCTAACTTCAGGAAT \\
\hline 45 & 12145 & TTACTGCTAAAATCCGAGGACAAAG & CTTGACAAAAGCCTAACATGTCACT \\
\hline 47 & 12777 & CAGTGTTTCAATAGACATGCCAAGA & CTGATTGATTACTGACTTGGTGTGG \\
\hline 49 & 1134 & CGTTTCACAAGGAAGGAATCA & TTTCGACATTTTGTCCGTTTC \\
\hline 50 & 3101 & TGTCGAACAGATCCGTCACTTGTAA & TGTGTCTCAGACCTTTCTTGTCGTG \\
\hline 53 & 9100 & ACAAGAATTCCCACGCCTTAT & ACGGTTATGTCGAAGATGTCG \\
\hline 56 & 10282 & GTATCAAGTGCTTCCTTTCCTCGTC & AGAACGAACCGTTTTCGTACCATTA \\
\hline 57 & 1793 & AGTTTGGGATGTAACGATGCAAGAT & CACCAAAGACAGACTGGTGCATATC \\
\hline 58 & 2626 & CAAATTACTCGAGTCCGACACAATG & AGCCGGTATATTCATGTTTGCTGAT \\
\hline 59 & 5750 & TCGGCCAGTACTTTGAACTTTCTTC & ATAATGTTGCAACCGCTTTGTTTCT \\
\hline 61 & 604 & GTGGTTTTGTCCCATTGAATGTCTAC & GACTTGCGAGACTTTATCGTCAACTG \\
\hline 62 & 954 & AGAGATGACCTCACTTTACGCAACTG & GAACATACAGGTTCACATCGCTGATT \\
\hline 63 & 2283 & CTAGCTTCTCTTCACGAGTCTCATCG & GCTGTCCTTTATTCCAGACGTATTCAG \\
\hline 64 & 3946 & CGTCCTACTAAAGGAAGCCTCTCAAA & ATAAGACAAAGCAGATGGAAGTGGTG \\
\hline 65 & 9509 & ATGACCTCCTCAACACCTTCAGTTC & AGTCTGTGTAATGGGCACCACTCTT \\
\hline 66 & 11416 & GTACCAAATTAATCCCAGATCGCTGT & TGTAGATCGAGACCAAGTCCTTTCTTC \\
\hline $\mathrm{X} 1$ & 17 & ATCTTCGTTTTCGTTGTCGCTCTC & CAGTCTTGAGTCCATCATCCAGGTT \\
\hline $\mathrm{X} 2$ & 190 & TGGGTTTTACTTCTTGTGGGTGTTG & GTGTTGCAATCCTTCTTGAACGTCT \\
\hline $\mathrm{X} 3$ & 908 & GTGTTATCGGTGCCGTCTCATACTC & CTGCTTATTCTCTTCACGGACCTCA \\
\hline $\mathrm{X} 5$ & 11882 & ACTGCAACTCCAACTACATCGAACC & GCAATTGTCCCGTAACTCTCAACAC \\
\hline $\mathrm{X} 6$ & 4742 & TGAGGGTGTGTACAATACGTTCGTT & GATGTTTCATGGGATGTCGTCGTAG \\
\hline $\mathrm{X} 7$ & 12667 & CATGTGGGAGAAAATCGATACGAGA & TACAACGGTTTGAGGTCCAGACTTG \\
\hline
\end{tabular}




\section{A. CLONES AND PRIMER}

Table A.4: Primer for non overlapping fragments of RNAseq in situ screen candidates

\begin{tabular}{|c|c|c|}
\hline au2ID & $\mathrm{Nr}$ & Sequence \\
\hline 4667 & 16_FWDT7 & AATAATACGACTCACTATAGGAGGTGCGCTGTCAACTATTACGAGT \\
\hline 4667 & 16_REVT7 & AATAATACGACTCACTATAGGTCGCACTTATCTCCGGTGACTCCGG \\
\hline 7373 & 26_FWDT7 & AATAATACGACTCACTATAGGGTGCGGAAAGTCCTTCAAGCGCTCG \\
\hline 7373 & 26_REVT7 & AATAATACGACTCACTATAGGGCACACGAACTGCTTCTCGCCGCCC \\
\hline 8329 & 30_FWDT7 & AATAATACGACTCACTATAGGGAGACCTGGGGAACACCACCACGGG \\
\hline 8329 & 30_REVT7 & AATAATACGACTCACTATAGGCCTCTTCATGGTGGTGTTCGGGGCG \\
\hline 8732 & 34_FWDT7 & AATAATACGACTCACTATAGGCAGCCCCCAGGGGCACGGAACAGCC \\
\hline 8732 & 34_REVT7 & AATAATACGACTCACTATAGGTTGCTGAATTCTCGCACAAGGAGGC \\
\hline
\end{tabular}




\section{Appendix B}

\section{RNAseq}

\section{B.1 Candidate Genes}

The following tables contain the candidate genes from the Venn diagrams (figure 4.10). "au2ID" are the au2 gene set identifiers used in the RNAseq analysis. "TcasID" are the gene identifiers from the older Tcas3.0 gene set. "FBgnID" are the flybase identifiers (including link to FB) of the corresponding ortho/homologs. "Symbol" are the gene symbols from flybase. "fC" are fold change values when comparing treatment (arrow ${ }^{R N A i}$, hedgehog ${ }^{R N A i}$ ) to wild type. "FDR" is the false discovery rate (Benjamini Hochberg adjusted p-values).

Table B.1: Posterior Wnt targets

\begin{tabular}{|c|c|c|c|c|c|}
\hline au2ID & TcasID & FBgnID & Symbol & fC & FDR \\
\hline au2.g10073.t1 & TC012363 & FBgn0030616 & $R p L 37 a$ & 0.37 & $3.38 \mathrm{E}-007$ \\
\hline au2.g10180.t1 & TC012432 & FBgn0040342 & $N A$ & 0.40 & $9.62 \mathrm{E}-011$ \\
\hline au2.g10495.t1 & TC012620 & NA & $N A$ & 0.30 & $5.55 \mathrm{E}-024$ \\
\hline au2.g10592.t1 & TC011826 & FBgn0039844 & $C G 160^{r}$ & 0.25 & $4.68 \mathrm{E}-021$ \\
\hline au2.g10685.t1 & TC011748 & FBgn0011278 & lbe & 0.12 & $6.27 \mathrm{E}-009$ \\
\hline au2.g10873.t1 & TC012851 & FBgn0001168 & $h$ & 0.33 & $4.76 \mathrm{E}-041$ \\
\hline au2.g11365.t1 & TC005856 & FBgn0262954 & $R p b 12$ & 0.47 & $1.51 \mathrm{E}-007$ \\
\hline au2.g11387.t1 & TC005841 & FBgn0000409 & $C y t-c-p$ & 0.46 & $3.16 \mathrm{E}-013$ \\
\hline
\end{tabular}


Table B.1 - continued from previous page

\begin{tabular}{|c|c|c|c|c|c|}
\hline au2ID & TcasID & FBgnID & Symbol & $\mathrm{fC}$ & FDR \\
\hline au2.g11461.t1 & TC005792 & FBgn0013325 & $R p L 11$ & 0.46 & 4.79E-010 \\
\hline au2.g11465.t1 & TC005785 & FBgn0004893 & bowl & 0.28 & 5.32E-027 \\
\hline au2.g11578.t1 & TC006413 & FBgn0031645 & CG3036 & 0.23 & $1.71 \mathrm{E}-030$ \\
\hline au2.g11681.t1 & TC005648 & FBgn0037686 & $R p L 34 b$ & 0.42 & $1.73 \mathrm{E}-008$ \\
\hline au2.g11821.t1 & TC006567 & FBgn0261608 & $R p L 37 A$ & 0.48 & 4.91E-006 \\
\hline au2.g11882.t1 & TC005505 & NA & $N A$ & 0.47 & $2.44 \mathrm{E}-017$ \\
\hline au2.g12008.t1 & TC006707 & FBgn0039081 & Irk2 & 0.32 & $9.22 \mathrm{E}-014$ \\
\hline au2.g12141.t1 & TC006782 & FBgn0010408 & $R p S 9$ & 0.40 & $5.58 \mathrm{E}-012$ \\
\hline au2.g12331.t1 & TC011204 & FBgn0028697 & $R p L 15$ & 0.43 & $4.02 \mathrm{E}-013$ \\
\hline au2.g12498.t1 & TC011085 & FBgn0004403 & $R p S 14 a$ & 0.40 & $2.20 \mathrm{E}-011$ \\
\hline au2.g12523.t1 & ТC011406 & FBgn0000273 & $P k a-C 1$ & 0.48 & $1.46 \mathrm{E}-006$ \\
\hline au2.g1265.t1 & NA & NA & $N A$ & 0.32 & 2.93E-008 \\
\hline au2.g12777.t1 & NA & NA & $N A$ & 0.41 & 8.79E-018 \\
\hline au2.g1479.t1 & TC001756 & FBgn0011016 & SsRbeta & 0.41 & $2.96 \mathrm{E}-011$ \\
\hline au2.g1604.t1 & TC004178 & NA & $N A$ & 0.39 & $1.20 \mathrm{E}-010$ \\
\hline au2.g1783.t1 & TC002098 & FBgn0037328 & $R p L 35 A$ & 0.44 & $1.18 \mathrm{E}-008$ \\
\hline au2.g1826.t1 & TC016001 & $\mathrm{NA}$ & $N A$ & 0.42 & $1.57 \mathrm{E}-026$ \\
\hline au2.g1922.t1 & $\mathrm{NA}$ & $\mathrm{NA}$ & $N A$ & 0.42 & $1.22 \mathrm{E}-016$ \\
\hline au2.g1949.t1 & TC001524 & FBgn0086710 & $R p L 30$ & 0.38 & 2.77E-009 \\
\hline au2.g216.t1 & $\mathrm{NA}$ & $\mathrm{NA}$ & $N A$ & 0.23 & 8.35E-019 \\
\hline au2.g219.t1 & TC015494 & FBgn0023214 & $e d l$ & 0.49 & $1.76 \mathrm{E}-014$ \\
\hline au2.g2239.t1 & NA & FBgn0038834 & $R p S 30$ & 0.42 & $4.08 \mathrm{E}-009$ \\
\hline au2.g2250.t1 & TC004892 & FBgn0038834 & $R p S 30$ & 0.41 & 1.69E-009 \\
\hline au2.g2324.t1 & TC004444 & FBgn0063492 & GstE8 & 0.38 & 4.12E-019 \\
\hline au2.g2437.t1 & TC000813 & NA & $N A$ & 0.38 & $1.90 \mathrm{E}-018$ \\
\hline au2.g2544.t1 & TC000868 & FBgn0004858 & $e l B$ & 0.20 & $1.04 \mathrm{E}-080$ \\
\hline au2.g2727.t1 & TC000592 & NA & $N A$ & 0.27 & $1.68 \mathrm{E}-040$ \\
\hline au2.g279.t1 & TC015179 & FBgn0037723 & $S p d S$ & 0.44 & 5.62E-008 \\
\hline au2.g3132.t1 & TC000355 & FBgn0029868 & $C G 3446$ & 0.48 & 2.97E-007 \\
\hline
\end{tabular}


Table B.1 - continued from previous page

\begin{tabular}{|c|c|c|c|c|c|}
\hline $\mathrm{au} 2 \mathrm{ID}$ & TcasID & FBgnID & Symbol & $\mathrm{fC}$ & FDR \\
\hline au2.g3150.t1 & TC001225 & FBgn0003942 & $R p S 27 A$ & 0.44 & $4.82 \mathrm{E}-008$ \\
\hline au2.g3254.t1 & $\mathrm{NA}$ & NA & $N A$ & 0.28 & $9.77 \mathrm{E}-035$ \\
\hline au2.g3381.t1 & TC000180 & FBgn0032518 & RpL24 & 0.46 & $8.42 \mathrm{E}-009$ \\
\hline au2.g3484.t1 & TC002331 & FBgn0033961 & $C G 12859$ & 0.49 & $9.46 \mathrm{E}-006$ \\
\hline au2.g3829.t1 & TC003304 & FBgn0031066 & CoVIb & 0.46 & $1.07 \mathrm{E}-010$ \\
\hline au2.g491.t1 & ТC015654 & FBgn0015288 & $R p L 22$ & 0.44 & $2.60 \mathrm{E}-011$ \\
\hline au2.g5142.t1 & TC003967 & FBgn0017579 & $R p L 14$ & 0.42 & $3.77 \mathrm{E}-010$ \\
\hline au2.g5337.t1 & NA & $\mathrm{NA}$ & $N A$ & 0.49 & $5.54 \mathrm{E}-005$ \\
\hline au2.g5437.t1 & TC007795 & FBgn0031980 & $R p L 36 A$ & 0.44 & 7.40E-008 \\
\hline au2.g5472.t1 & TC007682 & FBgn0052238 & CG32238 & 0.46 & 2.63E-006 \\
\hline au2.g5557.t1 & TC007636 & FBgn0031505 & $C G 12400$ & 0.46 & 7.73E-008 \\
\hline au2.g5635.t1 & ТC007576 & FBgn0000251 & $c a d$ & 0.19 & $9.28 \mathrm{E}-092$ \\
\hline au2.g5804.t1 & $\mathrm{NA}$ & FBgn0032694 & MESR3 & 0.41 & $5.60 \mathrm{E}-020$ \\
\hline au2.g6369.t1 & TC008413 & FBgn0031645 & CG3036 & 0.44 & $4.21 \mathrm{E}-010$ \\
\hline au2.g6853.t1 & ТC013812 & FBgn0035280 & Cpr62Bb & 0.39 & $3.75 \mathrm{E}-007$ \\
\hline au2.g6993.t1 & TC013723 & FBgn0013753 & $B g b$ & 0.17 & $1.56 \mathrm{E}-106$ \\
\hline au2.g6995.t1 & TC014076 & FBgn0011723 & byn & 0.07 & $6.16 \mathrm{E}-044$ \\
\hline au2.g7373.t1 & ТC013474 & FBgn0002573 & sens & 0.18 & $2.36 \mathrm{E}-047$ \\
\hline au2.g7499.t1 & TC014405 & FBgn0010411 & $R p S 18$ & 0.43 & $2.56 \mathrm{E}-008$ \\
\hline au2.g7550.t1 & TC013365 & FBgn0010638 & Sec61beta & 0.42 & 3.72E-010 \\
\hline au2.g7723.t1 & ТC014598 & FBgn0003900 & twi & 0.39 & $4.59 \mathrm{E}-016$ \\
\hline au2.g7751.t1 & TC013195 & FBgn0053511 & CG33511 & 0.33 & $3.39 \mathrm{E}-010$ \\
\hline au2.g7815.t1 & TC013163 & FBgn0000411 & $D$ & 0.33 & $9.01 \mathrm{E}-043$ \\
\hline au2.g7865.t1 & TC014685 & FBgn0050045 & Cpr $49 A a$ & 0.25 & $6.31 \mathrm{E}-014$ \\
\hline au2.g791.t1 & TC014844 & NA & $N A$ & 0.42 & $2.80 \mathrm{E}-005$ \\
\hline au2.g8207.t1 & NA & FBgn0052495 & CG32495 & 0.34 & $1.15 \mathrm{E}-016$ \\
\hline au2.g8303.t1 & TC009469 & FBgn0000606 & eve & 0.25 & $2.20 \mathrm{E}-067$ \\
\hline au2.g8329.t1 & TC009480 & NA & $N A$ & 0.23 & $5.82 \mathrm{E}-029$ \\
\hline au2.g8342.t1 & ТC009485 & FBgn0010078 & RpL23 & 0.46 & $3.99 \mathrm{E}-009$ \\
\hline
\end{tabular}


Table B.1 - continued from previous page

\begin{tabular}{|c|c|c|c|c|c|}
\hline au2ID & TcasID & FBgnID & Symbol & $\mathrm{fC}$ & FDR \\
\hline au2.g8382.t1 & TC009307 & NA & $N A$ & 0.41 & $5.37 \mathrm{E}-014$ \\
\hline au2.g8413.t1 & TC009288 & NA & $N A$ & 0.43 & $1.26 \mathrm{E}-018$ \\
\hline au2.g8461.t1 & TC009260 & FBgn & $R p S 12$ & 0.43 & $1.01 \mathrm{E}-007$ \\
\hline au2.g8732.t1 & TC015888 & $\mathrm{NA}$ & $N A$ & 0.44 & $3.54 \mathrm{E}-006$ \\
\hline au2.g9275.t1 & NA & FBgn0086472 & $R p S 25$ & 0.50 & $1.04 \mathrm{E}-005$ \\
\hline au2.g9928.t1 & TC004308 & FBgn0015521 & $R p S 21$ & 0.44 & $2.67 \mathrm{E}-008$ \\
\hline
\end{tabular}

Table B.2: Anterior Wnt targets

\begin{tabular}{|c|c|c|c|c|c|}
\hline au2ID & TcasID & FBgnID & Symbol & $\mathrm{fC}$ & FDR \\
\hline au2.g10282.t1 & ТC011993 & NA & $N A$ & 0.43 & $3.89 \mathrm{E}-005$ \\
\hline au2.g1134.t1 & TC004646 & NA & $N A$ & 0.37 & $2.24 \mathrm{E}-030$ \\
\hline au2.g1793.t1 & TC005250 & FBgn0000052 & ade2 & 0.45 & $1.51 \mathrm{E}-025$ \\
\hline au2.g2626.t1 & TC000937 & FBgn0000078 & Amy-d & 0.33 & $2.79 \mathrm{E}-011$ \\
\hline au2.g2627.t1 & TC000638 & FBgn0020506 & Amyrel & 0.28 & 3.11E-009 \\
\hline au2.g3101.t1 & TC001186 & FBgn0044028 & Notum & 0.43 & 3.31E-022 \\
\hline au2.g5750.t1 & TC007996 & FBgn0031514 & $N A$ & 0.26 & $1.06 \mathrm{E}-032$ \\
\hline au2.g5990.t1 & TC008176 & FBgn0019650 & toy & 0.39 & 4.91E-007 \\
\hline
\end{tabular}

Table B.3: Posterior hedgehog targets

\begin{tabular}{|c|c|c|c|c|c|}
\hline au2ID & TcasID & FBgnID & Symbol & $\mathrm{fC}$ & FDR \\
\hline au2.g10304.t1 & TC012497 & FBgn0015032 & Сур4с3 & 0.20 & 0.037 \\
\hline au2.g10402.t1 & TC012546 & FBgn0032219 & $C G 4995$ & 0.20 & 0.001 \\
\hline au2.g10490.t1 & TC012616 & FBgn0031294 & $I A-2$ & 0.40 & 0.019 \\
\hline
\end{tabular}


Table B. 3 - continued from previous page

\begin{tabular}{|c|c|c|c|c|c|}
\hline au2ID & TcasID & FBgnID & Symbol & $\mathrm{fC}$ & FDR \\
\hline au2.g10496.t1 & TC011888 & NA & $N A$ & 0.37 & 0.016 \\
\hline au2.g10497.t1 & NA & NA & $N A$ & 0.43 & 0.073 \\
\hline au2.g1056.t1 & TC013596 & FBgn0000116 & Argk & 0.43 & 0.001 \\
\hline au2.g10672.t1 & TC011760 & NA & $N A$ & 0.14 & $5.37 \mathrm{E}-005$ \\
\hline au2.g10678.t1 & TC011756 & FBgn0038966 & pinta & 0.38 & 0.047 \\
\hline au2.g10709.t1 & TC012758 & FBgn0025697 & $\begin{array}{l}\text { santa- } \\
\text { maria }\end{array}$ & 0.33 & 0.003 \\
\hline au2.g10716.t1 & NA & FBgn0025697 & $\begin{array}{l}\text { santa- } \\
\text { maria }\end{array}$ & 0.34 & 0.002 \\
\hline au2.g10739.t1 & TC011714 & FBgn0052473 & $C G 32473$ & 0.28 & 0.004 \\
\hline au2.g11036.t1 & NA & NA & $N A$ & 0.12 & $3.36 \mathrm{E}-006$ \\
\hline au2.g11038.t1 & TC006091 & NA & $N A$ & 0.23 & 0.017 \\
\hline au2.g11146.t1 & TC005995 & FBgn0025866 & CalpB & 0.43 & 0.044 \\
\hline au2.g11302.t1 & TC006255 & FBgn0265137 & Spn $42 D a$ & 0.42 & 0.012 \\
\hline au2.g11360.t1 & TC005860 & FBgn0030968 & $C G 7322$ & 0.39 & 0.048 \\
\hline au2.g11652.t1 & TC005669 & FBgn0036995 & $N A$ & 0.36 & 0.002 \\
\hline au2.g12010.t1 & TC005437 & FBgn0034394 & $C G 15096$ & 0.31 & 0.034 \\
\hline au2.g12107.t1 & TC005380 & NA & $N A$ & 0.31 & 0.017 \\
\hline au2.g12145.t1 & TC005344 & FBgn0264493 & $r d x$ & 0.39 & 0.023 \\
\hline au2.g12173.t1 & TC005327 & FBgn0050106 & CCHa1r & 0.30 & 0.019 \\
\hline au2.g12247.t1 & TC012946 & $\mathrm{NA}$ & $N A$ & 0.31 & 0.001 \\
\hline au2.g12667.t1 & TC011481 & NA & $N A$ & 0.32 & 0.008 \\
\hline au2.g1272.t1 & TC010864 & FBgn0031538 & $N A$ & 0.38 & 0.015 \\
\hline au2.g1382.t1 & TC004597 & FBgn0029896 & CG3168 & 0.32 & 0.004 \\
\hline au2.g17.t1 & TC015373 & NA & $N A$ & 0.44 & 0.047 \\
\hline au2.g190.t1 & TC015478 & NA & $N A$ & 0.32 & 0.008 \\
\hline au2.g1938.t1 & TC005191 & FBgn0036857 & CG9629 & 0.34 & 0.001 \\
\hline au2.g216.t1 & NA & NA & $N A$ & 0.50 & 0.033 \\
\hline au2.g257.t1 & TC015522 & FBgn0032287 & $C G 6415$ & 0.41 & 0.033 \\
\hline
\end{tabular}


Table B.3 - continued from previous page

\begin{tabular}{|c|c|c|c|c|c|}
\hline au2ID & TcasID & FBgnID & Symbol & $\mathrm{fC}$ & FDR \\
\hline au2.g2727.t1 & TC000592 & $\mathrm{NA}$ & $N A$ & 0.48 & 0.057 \\
\hline au2.g279.t1 & ТC015179 & FBgn0037723 & $S p d S$ & 0.38 & 0.008 \\
\hline au2.g2852.t1 & TC000520 & FBgn0003495 & $s p z$ & 0.36 & 0.010 \\
\hline au2.g2952.t1 & TC000459 & FBgn0033913 & $C G 8468$ & 0.45 & 0.031 \\
\hline au2.g3393.t1 & $\mathrm{NA}$ & FBgn0038149 & $N A$ & 0.37 & 0.043 \\
\hline au2.g3395.t1 & NA & FBgn0038149 & $N A$ & 0.25 & 0.022 \\
\hline au2.g3396.t1 & NA & FBgn0038149 & $N A$ & 0.25 & 0.022 \\
\hline au2.g3415.t1 & TC000174 & FBgn0038149 & $N A$ & 0.38 & 0.023 \\
\hline au2.g3417.t1 & TC000172 & FBgn0038149 & $N A$ & 0.23 & 0.010 \\
\hline au2.g3418.t1 & $\mathrm{NA}$ & FBgn0038149 & $N A$ & 0.27 & 0.024 \\
\hline au2.g3432.t1 & TC001375 & FBgn0051974 & $C G 31974$ & 0.34 & 0.010 \\
\hline au2.g3731.t1 & TC003228 & FBgn0036316 & CG10960 & 0.31 & 0.036 \\
\hline au2.g3759.t1 & TC003203 & FBgn0030574 & $C G 9413$ & 0.39 & 0.024 \\
\hline au2.g4120.t1 & TC002989 & FBgn0036169 & Fuca & 0.28 & 0.000 \\
\hline au2.g4122.t1 & TC002988 & FBgn0036169 & Fuca & 0.28 & 0.002 \\
\hline au2.g4461.t1 & TC000091 & FBgn0025592 & Gyk & 0.49 & 0.068 \\
\hline au2.g4667.t1 & TC010540 & FBgn0002528 & LanB2 & 0.40 & 0.000 \\
\hline au2.g4688.t1 & TC010496 & FBgn0037801 & CG3999 & 0.35 & 0.000 \\
\hline au2.g46.t1 & TC015389 & FBgn0038695 & $N A$ & 0.20 & 0.000 \\
\hline au2.g4742.t1 & TC003773 & FBgn0030334 & Karl & 0.31 & 0.016 \\
\hline au2.g505.t1 & TC015039 & NA & $N A$ & 0.30 & 0.050 \\
\hline au2.g5092.t1 & TC003919 & NA & $N A$ & 0.29 & 0.020 \\
\hline au2.g5115.t1 & ТC003939 & NA & $N A$ & 0.36 & 0.035 \\
\hline au2.g5129.t1 & TC002464 & FBgn0027611 & $L M 408$ & 0.31 & 0.000 \\
\hline au2.g5536.t1 & $\begin{array}{l}\text { TC007646- } \\
\text { GA }\end{array}$ & FBgn0085424 & $n u b$ & 0.45 & 0.047 \\
\hline au2.g5809.t1 & TC008043 & FBgn0026403 & $N d g$ & 0.30 & 0.000 \\
\hline au2.g5840.t1 & TC008063 & FBgn0004567 & slp2 & 0.38 & 0.050 \\
\hline
\end{tabular}


Table B. 3 - continued from previous page

\begin{tabular}{|c|c|c|c|c|c|}
\hline au2ID & TcasID & FBgnID & Symbol & $\mathrm{fC}$ & FDR \\
\hline au2.g5953.t1 & TC007351 & FBgn0026721 & $\begin{array}{l}\text { fat- } \\
\text { spondin }\end{array}$ & 0.33 & 0.006 \\
\hline au2.g6037.t1 & TC008202 & FBgn0031381 & Npc2a & 0.34 & 0.011 \\
\hline au2.g6199.t1 & TC007187 & FBgn0051871 & CG31871 & 0.26 & 0.000 \\
\hline au2.g6283.t1 & TC007141 & FBgn0000242 & $B x$ & 0.34 & 0.011 \\
\hline au2.g6578.t1 & TC016044 & FBgn0000313 & $\operatorname{chp}$ & 0.35 & 0.001 \\
\hline au2.g6598.t1 & TC002354 & FBgn0261243 & $P s a$ & 0.49 & 0.040 \\
\hline au2.g6712.t1 & TC010939 & FBgn0035665 & Jon65Aiii & 0.30 & 0.066 \\
\hline au2.g6823.t1 & TC013978 & NA & $N A$ & 0.25 & $3.07 \mathrm{E}-005$ \\
\hline au2.g6909.t1 & TC014022 & FBgn0035575 & $N A$ & 0.33 & 0.004 \\
\hline au2.g7000.t1 & TC013718 & FBgn0024150 & $A c^{r} 78 C$ & 0.37 & 0.019 \\
\hline au2.g7384.t1 & TC013464 & FBgn0031913 & $C G 5958$ & 0.43 & 0.036 \\
\hline au2.g7869.t1 & TC013128 & FBgn0050045 & Cpr49Aa & 0.46 & 0.078 \\
\hline au2.g7901.t1 & TC014706 & FBgn0033629 & Tsp47F & 0.28 & 0.007 \\
\hline au2.g7984.t1 & TC013049 & FBgn0038257 & smp-30 & 0.33 & 0.001 \\
\hline au2.g832.t1 & TC011529 & FBgn0040322 & $G N B P 2$ & 0.40 & 0.073 \\
\hline au2.g8486.t1 & TC009570 & FBgn0037736 & $N A$ & 0.21 & 0.071 \\
\hline au2.g8500.t1 & TC009233 & $\mathrm{NA}$ & $N A$ & 0.20 & $3.28 \mathrm{E}-005$ \\
\hline au2.g8529.t1 & TC009593 & FBgn0034999 & CG3394 & 0.31 & 0.001 \\
\hline au2.g9014.t1 & TC009808 & FBgn0041630 & Hexo1 & 0.39 & 0.006 \\
\hline au2.g908.t1 & TC001613 & FBgn0036756 & $\operatorname{cln} 3$ & 0.44 & 0.049 \\
\hline au2.g9100.t1 & TC009861 & FBgn0003174 & pwn & 0.44 & 0.012 \\
\hline au2.g9261.t1 & TC008883 & FBgn0086909 & CG31751 & 0.35 & 0.025 \\
\hline au2.g9385.t1 & TC008806 & NA & $N A$ & 0.35 & 0.006 \\
\hline au2.g9440.t1 & TC008778 & FBgn0041630 & Hexo1 & 0.32 & 0.001 \\
\hline au2.g9492.t1 & TC008734 & FBgn0039527 & $C G 5639$ & 0.49 & 0.011 \\
\hline au2.g9629.t1 & TC010171 & FBgn0037215 & $C G 12582$ & 0.31 & 0.022 \\
\hline au2.g9865.t1 & TC012282 & FBgn0040251 & $U g t 86 D i$ & 0.47 & 0.072 \\
\hline au2.g9867.t1 & TC012285 & FBgn0040257 & $U g t 86 D c$ & 0.36 & 0.001 \\
\hline
\end{tabular}


Table B.3 - continued from previous page

\begin{tabular}{|c|c|c|c|c|c|}
\hline au2ID & TcasID & FBgnID & Symbol & fC & FDR \\
\hline au2.g9915.t1 & TC012152 & FBgn0032612 & $C G 13282$ & 0.35 & 0.070 \\
\hline au2.g993.t1 & TC014177 & FBgn0036975 & $C G 5618$ & 0.40 & 0.015 \\
\hline
\end{tabular}

Table B.4: Anterior hedgehog targets

\begin{tabular}{|c|c|c|c|c|c|}
\hline au2ID & TcasID & FBgnID & Symbol & $\mathrm{fC}$ & FDR \\
\hline au2.g11416.t1 & TC005822 & NA & $N A$ & 0.38 & 0.044 \\
\hline au2.g2229.t1 & ТC004494 & FBgn0034093 & $N A$ & 0.23 & 0.023 \\
\hline au2.g2283.t1 & TC004922 & FBgn0043791 & $C G 8147$ & 0.48 & 0.070 \\
\hline au2.g2626.t1 & TC000937 & FBgn0000078 & Amy-d & 0.43 & 0.027 \\
\hline au2.g3946.t1 & TC003369 & FBgn0011576 & Cyp4d2 & 0.37 & 0.026 \\
\hline au2.g604.t1 & TC015713 & FBgn0030598 & $C G 9503$ & 0.42 & 0.035 \\
\hline au2.g9509.t1 & TC010105 & FBgn0011296 & l(2)efl & 0.31 & 0.005 \\
\hline au2.g954.t1 & TC004121 & FBgn0034089 & $N A$ & 0.50 & 0.078 \\
\hline
\end{tabular}

\section{B.2 Gene ontology enrichment}

The following tables contain all the significantly enriched GO terms found with the AMIGO term enrichment tool [Carbon et al., 2009]. Since in Tribolium only a small subset of genes has GO annotations, I used the annotations of Drosophila. Enrichment was only found in the posterior Wnt target gene set. This set contained 72 genes, however, for only 52 a Drosophila ortho/homolog was found. The tool compares the sample frequency, i.e. the frequency of a given GO term among the 52 genes of the target gene set, to the background frequency. The background frequency is the frequency of a given GO term in the whole Drosophila 


\section{B.2 Gene ontology enrichment}

genome. The calculated p-value is adjusted for multiple testing with the Bonferroni correction.

Table B.5: Biological Process

\begin{tabular}{|c|c|c|c|}
\hline GO term & $\begin{array}{l}\mathrm{P}- \\
\text { value }\end{array}$ & $\begin{array}{l}\text { Sample } \\
\text { frequency }\end{array}$ & $\begin{array}{l}\text { Background } \\
\text { frequency }\end{array}$ \\
\hline $\begin{array}{l}\text { GO: } 0034645 \text { cellular macromolecule } \\
\text { biosynthetic process }\end{array}$ & $4.67 \mathrm{e}-12$ & $\begin{array}{l}32 / 52 \\
(61.5 \%)\end{array}$ & $\begin{array}{l}1971 / 13796 \\
(14.3 \%)\end{array}$ \\
\hline $\begin{array}{l}\text { GO:0009059 macromolecule biosyn- } \\
\text { thetic process }\end{array}$ & $5.18 \mathrm{e}-12$ & $\begin{array}{l}32 / 52 \\
(61.5 \%)\end{array}$ & $\begin{array}{l}1978 / 13796 \\
(14.3 \%)\end{array}$ \\
\hline $\begin{array}{l}\text { GO:0000022 mitotic spindle elonga- } \\
\text { tion }\end{array}$ & $5.83 \mathrm{e}-12$ & $\begin{array}{l}11 / 52 \\
(21.2 \%)\end{array}$ & $80 / 13796(0.6 \%)$ \\
\hline GO:0051231 spindle elongation & $7.74 \mathrm{e}-12$ & $\begin{array}{l}11 / 52 \\
(21.2 \%)\end{array}$ & $82 / 13796(0.6 \%)$ \\
\hline $\begin{array}{l}\text { GO:0044249 cellular biosynthetic } \\
\text { process }\end{array}$ & $1.35 \mathrm{e}-11$ & $\begin{array}{l}34 / 52 \\
(65.4 \%)\end{array}$ & $\begin{array}{l}2374 / 13796 \\
(17.2 \%)\end{array}$ \\
\hline $\begin{array}{l}\text { GO:1901576 organic substance } \\
\text { biosynthetic process }\end{array}$ & $1.81 \mathrm{e}-11$ & $\begin{array}{l}34 / 52 \\
(65.4 \%)\end{array}$ & $\begin{array}{l}2397 / 13796 \\
(17.4 \%)\end{array}$ \\
\hline GO:0010467 gene expression & $3.42 \mathrm{e}-11$ & $\begin{array}{l}33 / 52 \\
(63.5 \%)\end{array}$ & $\begin{array}{l}2276 / 13796 \\
(16.5 \%)\end{array}$ \\
\hline GO:0009058 biosynthetic process & $3.49 \mathrm{e}-11$ & $\begin{array}{l}34 / 52 \\
(65.4 \%)\end{array}$ & $\begin{array}{l}2449 / 13796 \\
(17.8 \%)\end{array}$ \\
\hline GO:0006412 translation & $3.02 \mathrm{e}-09$ & $\begin{array}{l}20 / 52 \\
(38.5 \%)\end{array}$ & $800 / 13796(5.8 \%)$ \\
\hline $\begin{array}{l}\text { GO:0044237 cellular metabolic pro- } \\
\text { cess }\end{array}$ & $1.66 \mathrm{e}-08$ & $\begin{array}{l}41 / 52 \\
(78.8 \%)\end{array}$ & $\begin{array}{l}4580 / 13796 \\
(33.2 \%)\end{array}$ \\
\hline $\begin{array}{l}\text { GO:0007052 mitotic spindle organi- } \\
\text { zation }\end{array}$ & $2.62 \mathrm{e}-07$ & $\begin{array}{l}11 / 52 \\
(21.2 \%)\end{array}$ & $210 / 13796(1.5 \%)$ \\
\hline GO:0009987 cellular process & $9.21 \mathrm{e}-07$ & $\begin{array}{l}49 / 52 \\
(94.2 \%)\end{array}$ & $\begin{array}{l}7741 / 13796 \\
(56.1 \%)\end{array}$ \\
\hline GO:0007051 spindle organization & $1.60 \mathrm{e}-06$ & $\begin{array}{l}11 / 52 \\
(21.2 \%)\end{array}$ & $249 / 13796(1.8 \%)$ \\
\hline
\end{tabular}

Continued on next page 


\section{B. RNASEQ}

Table B.5 - continued from previous page

\begin{tabular}{|c|c|c|c|}
\hline GO term & $\begin{array}{l}\mathrm{P}- \\
\text { value }\end{array}$ & $\begin{array}{l}\text { Sample } \\
\text { frequency }\end{array}$ & $\begin{array}{l}\text { Background } \\
\text { frequency }\end{array}$ \\
\hline $\begin{array}{l}\text { GO:0044260 cellular macromolecule } \\
\text { metabolic process }\end{array}$ & $7.45 \mathrm{e}-06$ & $\begin{array}{l}33 / 52 \\
(63.5 \%)\end{array}$ & $\begin{array}{l}3507 / 13796 \\
(25.4 \%)\end{array}$ \\
\hline GO:0008152 metabolic process & $2.29 \mathrm{e}-05$ & $\begin{array}{l}42 / 52 \\
(80.8 \%)\end{array}$ & $\begin{array}{l}5916 / 13796 \\
(42.9 \%)\end{array}$ \\
\hline $\begin{array}{l}\text { GO:0000226 microtubule cytoskele- } \\
\text { ton organization }\end{array}$ & $2.58 \mathrm{e}-05$ & $\begin{array}{l}12 / 52 \\
(23.1 \%)\end{array}$ & $408 / 13796(3.0 \%)$ \\
\hline $\begin{array}{ll}\text { GO:0043170 } & \text { macromolecule } \\
\text { metabolic process } & \end{array}$ & $2.51 \mathrm{e}-04$ & $\begin{array}{l}34 / 52 \\
(65.4 \%)\end{array}$ & $\begin{array}{l}4238 / 13796 \\
(30.7 \%)\end{array}$ \\
\hline $\begin{array}{l}\text { GO:0044267 cellular protein } \\
\text { metabolic process }\end{array}$ & $3.23 \mathrm{e}-04$ & $\begin{array}{l}22 / 52 \\
(42.3 \%)\end{array}$ & $\begin{array}{l}1875 / 13796 \\
(13.6 \%)\end{array}$ \\
\hline $\begin{array}{l}\text { GO:0007017 microtubule-based } \\
\text { process }\end{array}$ & $3.46 \mathrm{e}-04$ & $\begin{array}{l}12 / 52 \\
(23.1 \%)\end{array}$ & $518 / 13796(3.8 \%)$ \\
\hline $\begin{array}{l}\text { GO:0007010 cytoskeleton organiza- } \\
\text { tion }\end{array}$ & $4.41 \mathrm{e}-04$ & $\begin{array}{l}13 / 52 \\
(25.0 \%)\end{array}$ & $637 / 13796(4.6 \%)$ \\
\hline $\begin{array}{l}\text { GO:0071704 organic substance } \\
\text { metabolic process }\end{array}$ & $1.31 \mathrm{e}-03$ & $\begin{array}{l}37 / 52 \\
(71.2 \%)\end{array}$ & $\begin{array}{l}5264 / 13796 \\
(38.2 \%)\end{array}$ \\
\hline $\begin{array}{l}\text { GO:0044238 primary metabolic } \\
\text { process }\end{array}$ & $3.25 \mathrm{e}-03$ & $\begin{array}{l}35 / 52 \\
(67.3 \%)\end{array}$ & $\begin{array}{l}4924 / 13796 \\
(35.7 \%)\end{array}$ \\
\hline $\begin{array}{l}\text { GO:0007442 hindgut morphogene- } \\
\text { sis }\end{array}$ & $3.56 \mathrm{e}-03$ & $5 / 52(9.6 \%)$ & $63 / 13796(0.5 \%)$ \\
\hline GO:0061525 hindgut development & $3.56 \mathrm{e}-03$ & $5 / 52(9.6 \%)$ & $63 / 13796(0.5 \%)$ \\
\hline GO:0000278 mitotic cell cycle & $4.53 \mathrm{e}-03$ & $\begin{array}{l}11 / 52 \\
(21.2 \%)\end{array}$ & $547 / 13796(4.0 \%)$ \\
\hline $\begin{array}{l}\text { GO:0048546 digestive tract mor- } \\
\text { phogenesis }\end{array}$ & $7.39 \mathrm{e}-03$ & $5 / 52(9.6 \%)$ & $73 / 13796(0.5 \%)$ \\
\hline $\begin{array}{l}\text { GO:0006366 transcription from } \\
\text { RNA polymerase II promoter }\end{array}$ & $8.00 \mathrm{e}-03$ & $\begin{array}{l}10 / 52 \\
(19.2 \%)\end{array}$ & $470 / 13796(3.4 \%)$ \\
\hline GO:0007369 gastrulation & $1.85 \mathrm{e}-02$ & $5 / 52(9.6 \%)$ & $88 / 13796(0.6 \%)$ \\
\hline
\end{tabular}




\section{B.2 Gene ontology enrichment}

Table B.5 - continued from previous page

\begin{tabular}{|c|c|c|c|}
\hline GO term & $\begin{array}{l}\mathrm{P}- \\
\text { value }\end{array}$ & $\begin{array}{l}\text { Sample } \\
\text { frequency }\end{array}$ & $\begin{array}{l}\text { Background } \\
\text { frequency }\end{array}$ \\
\hline $\begin{array}{l}\text { GO:0071822 protein complex sub- } \\
\text { unit organization }\end{array}$ & $2.47 \mathrm{e}-02$ & $\begin{array}{l}11 / 52 \\
(21.2 \%)\end{array}$ & $655 / 13796(4.7 \%)$ \\
\hline $\begin{array}{l}\text { GO:0019538 protein metabolic pro- } \\
\text { cess }\end{array}$ & $2.56 \mathrm{e}-02$ & $\begin{array}{l}22 / 52 \\
(42.3 \%)\end{array}$ & $\begin{array}{l}2427 / 13796 \\
(17.6 \%)\end{array}$ \\
\hline $\begin{array}{l}\text { GO:0006357 regulation of transcrip- } \\
\text { tion from RNA polymerase II pro- } \\
\text { moter }\end{array}$ & $2.83 \mathrm{e}-02$ & $9 / 52(17.3 \%)$ & $432 / 13796(3.1 \%)$ \\
\hline $\begin{array}{l}\text { GO:0048619 embryonic hindgut } \\
\text { morphogenesis }\end{array}$ & $4.72 \mathrm{e}-02$ & $4 / 52(7.7 \%)$ & $54 / 13796(0.4 \%)$ \\
\hline $\begin{array}{l}\text { GO:0055123 digestive system devel- } \\
\text { opment }\end{array}$ & $4.75 \mathrm{e}-02$ & $5 / 52(9.6 \%)$ & $107 / 13796(0.8 \%)$ \\
\hline $\begin{array}{l}\text { GO:0048565 digestive tract devel- } \\
\text { opment }\end{array}$ & $4.75 \mathrm{e}-02$ & $5 / 52(9.6 \%)$ & $107 / 13796(0.8 \%)$ \\
\hline $\begin{array}{l}\text { GO:0009880 embryonic pattern } \\
\text { specification }\end{array}$ & $5.99 \mathrm{e}-02$ & $7 / 52(13.5 \%)$ & $269 / 13796(1.9 \%)$ \\
\hline $\begin{array}{l}\text { GO:0043933 macromolecular com- } \\
\text { plex subunit organization }\end{array}$ & $6.17 \mathrm{e}-02$ & $\begin{array}{l}11 / 52 \\
(21.2 \%)\end{array}$ & $724 / 13796(5.2 \%)$ \\
\hline $\begin{array}{l}\text { GO:0045944 positive regulation of } \\
\text { transcription from RNA polymerase } \\
\text { II promoter }\end{array}$ & $7.68 \mathrm{e}-02$ & $6 / 52(11.5 \%)$ & $192 / 13796(1.4 \%)$ \\
\hline $\begin{array}{l}\text { GO:0044271 cellular nitrogen com- } \\
\text { pound biosynthetic process }\end{array}$ & $9.95 \mathrm{e}-02$ & $\begin{array}{l}14 / 52 \\
(26.9 \%)\end{array}$ & $\begin{array}{l}1205 / 13796 \\
(8.7 \%)\end{array}$ \\
\hline
\end{tabular}


Table B.6: Cellular Component

\begin{tabular}{|c|c|c|c|}
\hline GO term & $\begin{array}{l}\mathrm{P}- \\
\text { value }\end{array}$ & $\begin{array}{l}\text { Sample } \\
\text { frequency }\end{array}$ & $\begin{array}{l}\text { Background } \\
\text { frequency }\end{array}$ \\
\hline GO:0044391 ribosomal subunit & $3.66 \mathrm{e}-22$ & $\begin{array}{l}20 / 52 \\
(38.5 \%)\end{array}$ & $176 / 13796(1.3 \%)$ \\
\hline GO:0022626 cytosolic ribosome & $3.40 \mathrm{e}-21$ & $\begin{array}{l}20 / 52 \\
(38.5 \%)\end{array}$ & $196 / 13796(1.4 \%)$ \\
\hline GO:0044445 cytosolic part & $7.52 \mathrm{e}-20$ & $\begin{array}{l}20 / 52 \\
(38.5 \%)\end{array}$ & $228 / 13796(1.7 \%)$ \\
\hline GO:0005840 ribosome & $5.94 \mathrm{e}-18$ & $\begin{array}{l}20 / 52 \\
(38.5 \%)\end{array}$ & $283 / 13796(2.1 \%)$ \\
\hline $\begin{array}{l}\text { GO:0022625 cytosolic large riboso- } \\
\text { mal subunit }\end{array}$ & $1.97 \mathrm{e}-15$ & $\begin{array}{l}12 / 52 \\
(23.1 \%)\end{array}$ & $59 / 13796(0.4 \%)$ \\
\hline $\begin{array}{l}\text { GO:0030529 ribonucleoprotein } \\
\text { complex }\end{array}$ & $1.80 \mathrm{e}-12$ & $\begin{array}{l}21 / 52 \\
(40.4 \%)\end{array}$ & $620 / 13796(4.5 \%)$ \\
\hline $\begin{array}{l}\text { GO:0015934 large ribosomal sub- } \\
\text { unit }\end{array}$ & $4.30 \mathrm{e}-12$ & $\begin{array}{l}12 / 52 \\
(23.1 \%)\end{array}$ & $108 / 13796(0.8 \%)$ \\
\hline GO:0005829 cytosol & $1.40 \mathrm{e}-10$ & $\begin{array}{l}20 / 52 \\
(38.5 \%)\end{array}$ & $678 / 13796(4.9 \%)$ \\
\hline $\begin{array}{l}\text { GO:0022627 cytosolic small riboso- } \\
\text { mal subunit }\end{array}$ & $2.91 \mathrm{e}-09$ & $8 / 52(15.4 \%)$ & $43 / 13796(0.3 \%)$ \\
\hline GO:0043229 intracellular organelle & $4.03 \mathrm{e}-09$ & $\begin{array}{l}40 / 52 \\
(76.9 \%)\end{array}$ & $\begin{array}{l}4158 / 13796 \\
(30.1 \%)\end{array}$ \\
\hline GO:0043226 organelle & $4.90 \mathrm{e}-09$ & $\begin{array}{l}40 / 52 \\
(76.9 \%)\end{array}$ & $\begin{array}{l}4181 / 13796 \\
(30.3 \%)\end{array}$ \\
\hline $\begin{array}{l}\text { GO:0015935 small ribosomal sub- } \\
\text { unit }\end{array}$ & $2.77 \mathrm{e}-07$ & $8 / 52(15.4 \%)$ & $74 / 13796(0.5 \%)$ \\
\hline GO:0044424 intracellular part & $2.78 \mathrm{e}-07$ & $\begin{array}{l}42 / 52 \\
(80.8 \%)\end{array}$ & $\begin{array}{l}5228 / 13796 \\
(37.9 \%)\end{array}$ \\
\hline GO:0005622 intracellular & $5.34 \mathrm{e}-07$ & $\begin{array}{l}42 / 52 \\
(80.8 \%)\end{array}$ & $\begin{array}{l}5323 / 13796 \\
(38.6 \%)\end{array}$ \\
\hline
\end{tabular}


Table B.6 - continued from previous page

\begin{tabular}{|c|c|c|c|}
\hline GO term & $\begin{array}{l}\mathrm{P}- \\
\text { value }\end{array}$ & $\begin{array}{l}\text { Sample } \\
\text { frequency }\end{array}$ & $\begin{array}{l}\text { Background } \\
\text { frequency }\end{array}$ \\
\hline $\begin{array}{l}\text { GO:0044446 intracellular organelle } \\
\text { part }\end{array}$ & $5.61 \mathrm{e}-07$ & $\begin{array}{l}29 / 52 \\
(55.8 \%)\end{array}$ & $\begin{array}{l}2428 / 13796 \\
(17.6 \%)\end{array}$ \\
\hline GO:0044422 organelle part & $7.27 \mathrm{e}-07$ & $\begin{array}{l}29 / 52 \\
(55.8 \%)\end{array}$ & $\begin{array}{l}2454 / 13796 \\
(17.8 \%)\end{array}$ \\
\hline GO:0005737 cytoplasm & $3.50 \mathrm{e}-06$ & $\begin{array}{l}31 / 52 \\
(59.6 \%)\end{array}$ & $\begin{array}{l}3002 / 13796 \\
(21.8 \%)\end{array}$ \\
\hline $\begin{array}{l}\text { GO:0032991 macromolecular com- } \\
\text { plex }\end{array}$ & $3.68 \mathrm{e}-06$ & $\begin{array}{l}29 / 52 \\
(55.8 \%)\end{array}$ & $\begin{array}{l}2624 / 13796 \\
(19.0 \%)\end{array}$ \\
\hline $\begin{array}{l}\text { GO:0043228 non-membrane- } \\
\text { bounded organelle }\end{array}$ & $5.77 \mathrm{e}-06$ & $\begin{array}{l}22 / 52 \\
(42.3 \%)\end{array}$ & $\begin{array}{l}1503 / 13796 \\
(10.9 \%)\end{array}$ \\
\hline $\begin{array}{l}\text { GO:0043232 intracellular non- } \\
\text { membrane-bounded organelle }\end{array}$ & $5.77 \mathrm{e}-06$ & $\begin{array}{l}22 / 52 \\
(42.3 \%)\end{array}$ & $\begin{array}{l}1503 / 13796 \\
(10.9 \%)\end{array}$ \\
\hline GO:0044444 cytoplasmic part & $1.08 \mathrm{e}-05$ & $\begin{array}{l}27 / 52 \\
(51.9 \%)\end{array}$ & $\begin{array}{l}2376 / 13796 \\
(17.2 \%)\end{array}$ \\
\hline GO:0044464 cell part & $2.86 \mathrm{e}-05$ & $\begin{array}{l}42 / 52 \\
(80.8 \%)\end{array}$ & $\begin{array}{l}5954 / 13796 \\
(43.2 \%)\end{array}$ \\
\hline GO:0005623 cell & $2.86 \mathrm{e}-05$ & $\begin{array}{l}42 / 52 \\
(80.8 \%)\end{array}$ & $\begin{array}{l}5954 / 13796 \\
(43.2 \%)\end{array}$ \\
\hline
\end{tabular}

Table B.7: Molecular Function

\begin{tabular}{|c|c|c|c|}
\hline GO term & $\begin{array}{l}\mathrm{P}- \\
\text { value }\end{array}$ & $\begin{array}{l}\text { Sample } \\
\text { frequency }\end{array}$ & $\begin{array}{l}\text { Background } \\
\text { frequency }\end{array}$ \\
\hline $\begin{array}{l}\text { GO:0003735 structural constituent } \\
\text { of ribosome }\end{array}$ & $2.68 \mathrm{e}-18$ & $\begin{array}{l}20 / 52 \\
(38.5 \%)\end{array}$ & $272 / 13796(2.0 \%)$ \\
\hline $\begin{array}{l}\text { GO:0005198 structural molecule ac- } \\
\text { tivity }\end{array}$ & $7.13 \mathrm{e}-14$ & $\begin{array}{l}22 / 52 \\
(42.3 \%)\end{array}$ & $606 / 13796(4.4 \%)$ \\
\hline
\end{tabular}

Continued on next page 
Table B.7 - continued from previous page

\begin{tabular}{|c|c|c|c|}
\hline GO term & $\begin{array}{l}\mathrm{P}- \\
\text { value }\end{array}$ & $\begin{array}{l}\text { Sample } \\
\text { frequency }\end{array}$ & $\begin{array}{l}\text { Background } \\
\text { frequency }\end{array}$ \\
\hline $\begin{array}{l}\text { GO:0000981 sequence-specific DNA } \\
\text { binding RNA polymerase II tran- } \\
\text { scription factor activity }\end{array}$ & $3.17 \mathrm{e}-04$ & $8 / 52(15.4 \%)$ & $180 / 13796(1.3 \%)$ \\
\hline $\begin{array}{l}\text { GO:0003705 RNA polymerase II } \\
\text { distal enhancer sequence-specific } \\
\text { DNA binding transcription factor } \\
\text { activity }\end{array}$ & $6.44 \mathrm{e}-03$ & $5 / 52(9.6 \%)$ & $71 / 13796(0.5 \%)$ \\
\hline $\begin{array}{l}\text { GO:0003700 sequence-specific DNA } \\
\text { binding transcription factor activity }\end{array}$ & $4.18 \mathrm{e}-02$ & $9 / 52(17.3 \%)$ & $454 / 13796(3.3 \%)$ \\
\hline $\begin{array}{l}\text { GO:0001071 nucleic acid binding } \\
\text { transcription factor activity }\end{array}$ & $4.18 \mathrm{e}-02$ & $9 / 52(17.3 \%)$ & $454 / 13796(3.3 \%)$ \\
\hline
\end{tabular}




\section{B.3 Tc-senseless phylogenetic tree}

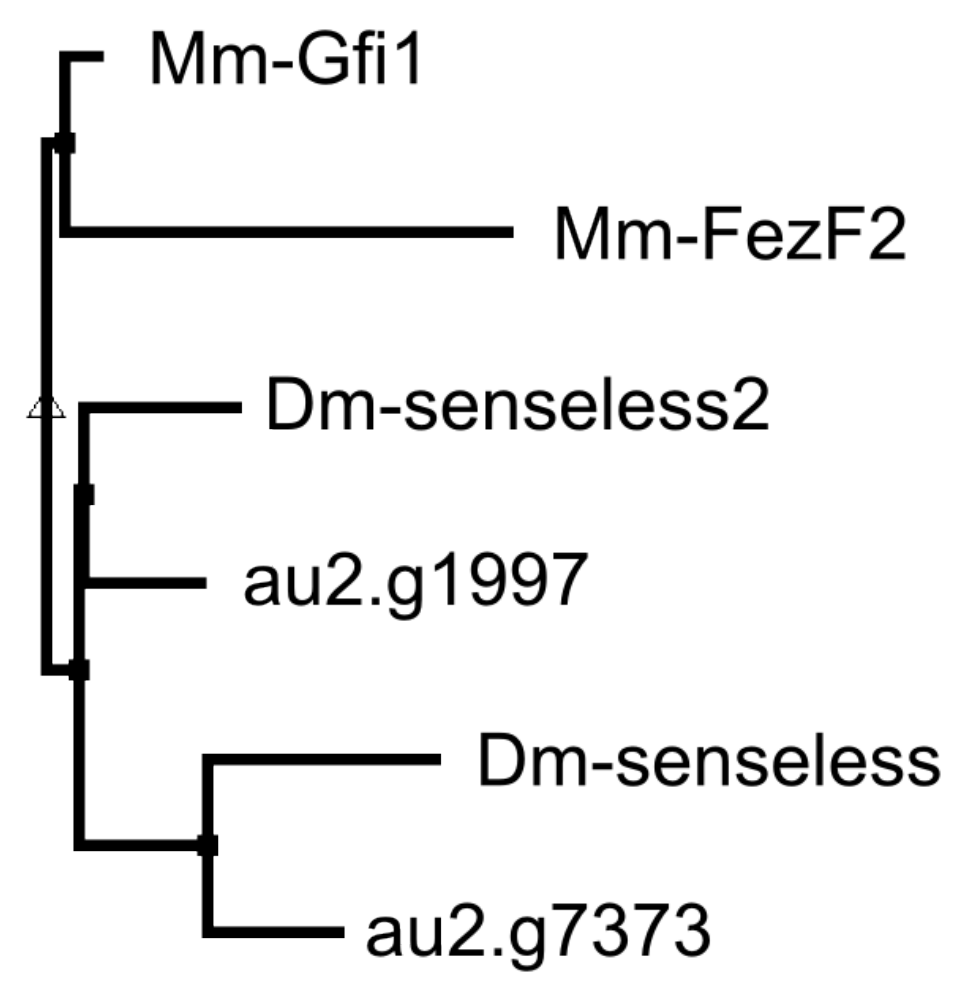

Figure B.1: Tc-senseless phylogenetic tree - Alignments of the best two hits of the gene au2.g7373 show that it is the ortholog of Dm-senseless. Sequences were identified using BLAST. Alignment and tree construction was done with Multalin [Corpet, 1988] 
B. RNASEQ 


\section{Appendix C}

\section{Scripts}

\section{C.1 R}

\section{C.1.1 DESeq Analysis}

This script contains all the relevant parameters which were used in the statistical analysis of the RNAseq data using the Tc-arrow ${ }^{R N A i}$ as example.

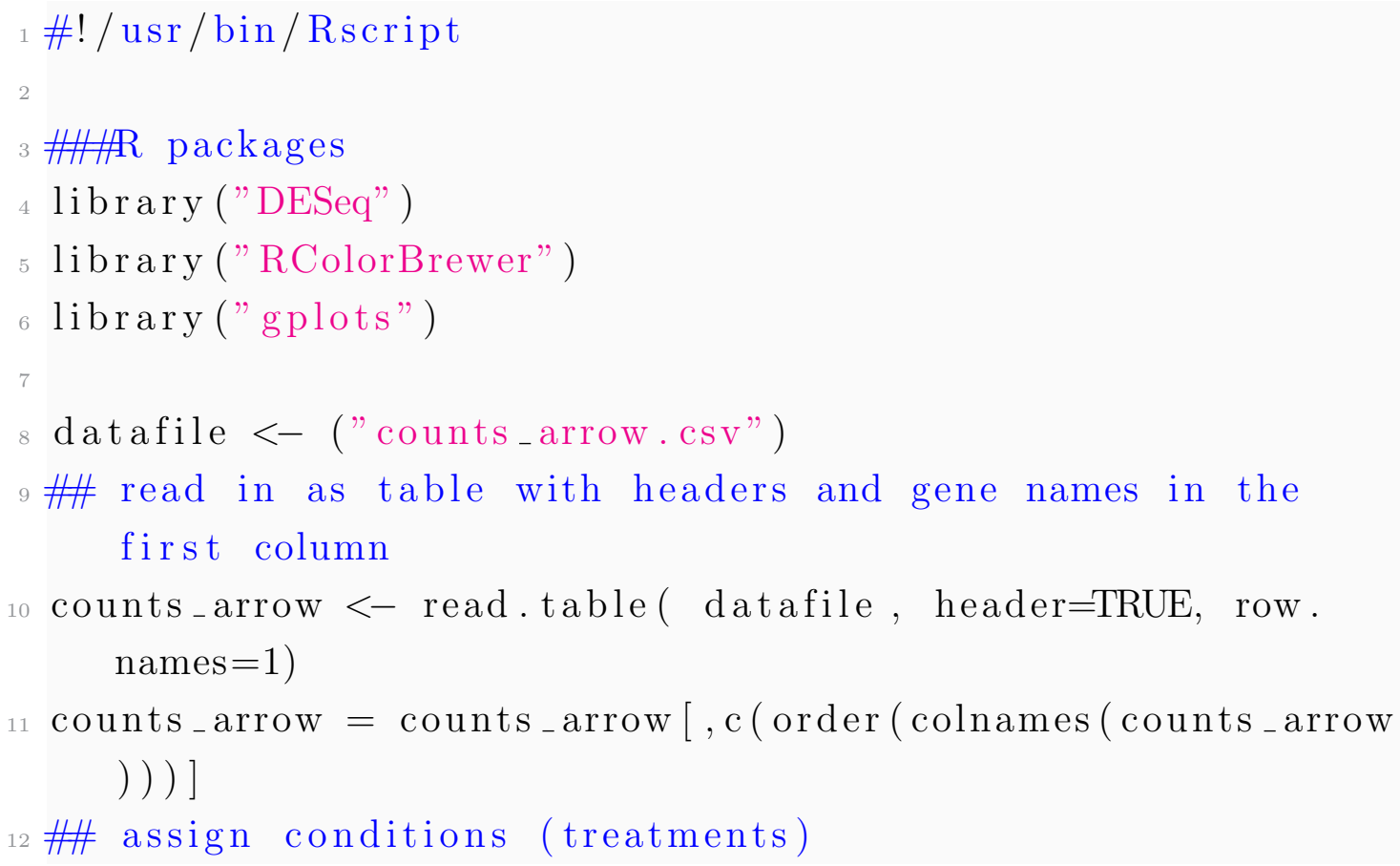




\section{SCRIPTS}

conds_arrow $=$ as. factor $\left(\operatorname{gsub}\left({ }^{\prime}[0-9]^{\prime},{ }^{\prime},{ }^{\prime}\right.\right.$, colnames $(\operatorname{counts}-$ arrow ), perl=T))

14

\#\#\#arrow

cds_arrow $<-$ newCountDataSet (counts_arrow, conds_arrow )

cds_arrow <- estimateSizeFactors ( cds_arrow )

cds_arrow $<-$ estimateDispersions ( cds_arrow )

pdf ( file=" DispersionPlot_arrow.pdf")

plotDispEsts ( cds_arrow)

dev. off ()

\#\#\#\#|\#CA

cdsBlind arrow $_{-}$estimateDispersions ( cds arrow $_{-}$method=" blind" )

${ }_{26}$ vsd $_{-}$arrow $=$varianceStabilizingTransformation ( cdsBlind arrow )

${ }_{27}$ dists_arrow $=\operatorname{dist}\left(\mathrm{t}\left(\operatorname{exprs}\left(\operatorname{vsd}_{-}\right.\right.\right.$arrow $\left.\left.)\right)\right)$

$\operatorname{pdf}(\mathrm{file}=$ "PCA_plot_arrow.pdf" )

plotPCA (vsd_arrow, intgroup="condition")

dev. off ()

\#\#\#\#heatmap

hmcol = colorRampPalette (brewer $\cdot \operatorname{pal}(9$, "GnBu" $))(100)$

mat $=$ as.matrix ( dists_arrow )

rownames $($ mat $)=$ colnames $($ mat $)=$ with $\left(\right.$ pData $\left(\operatorname{cdsBlind} \_\right.$arrow $)$

, paste(condition))

pdf ( file=" heatmap_arrow $\cdot$ pdf" )

heatmap.2(mat, trace="none", $\operatorname{col}=\operatorname{rev}($ hmcol $), \operatorname{margin}=\mathrm{c}$

$(13,13))$

dev. off ()

\#\#filtering independent

rs $=$ rowSums $\left(\operatorname{counts}\left(\operatorname{cds}_{-}\right.\right.$arrow $\left.)\right)$ 


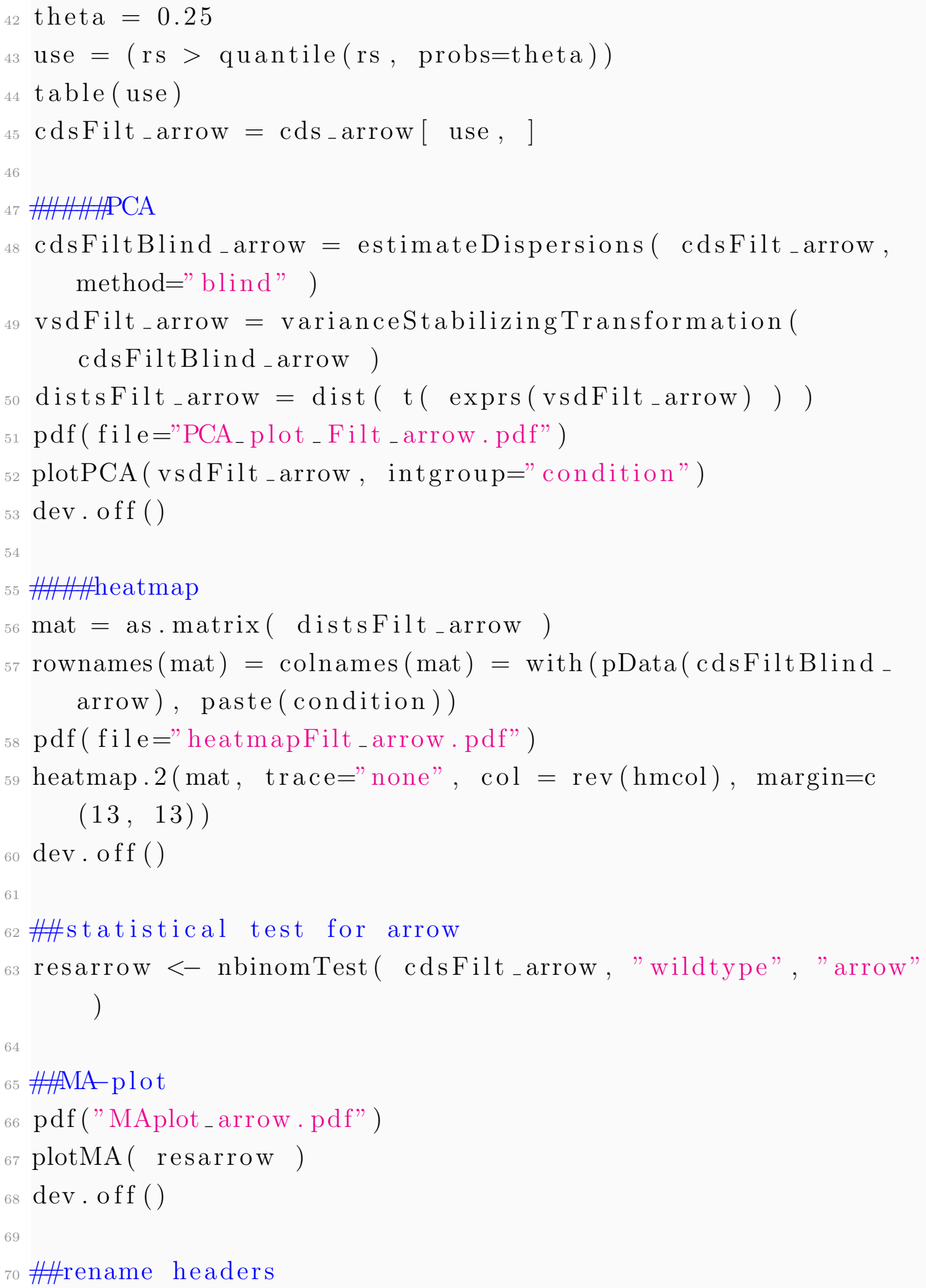




\section{SCRIPTS}

71 colnames $($ resarrow $)=c("$ id" , "arrowbaseMean" , " arrowbaseMeanA" , "arrowbaseMeanB" , "arrowfoldChange" , " arrowlog2FoldChange" , "arrowpval" , "arrowpadj")

72

\#\#significance criteria

74 resSigarrow $<-$ resarrow $[$ resarrow $\$$ arrowpadj $<0.1$, ]

5 \#\#up and down regulated

6 resSigarrowup <- resSigarrow [resSigarrow $\$$

arrowlog 2 FoldChange $>1$,

77 resSigarrowdown $<-$ resSigarrow [resSigarrow $\$$

arrowlog 2 FoldChange $<-1$, ]

78 \#\#)utput data tables

79 write.csv( resarrow, file="resarrow.csv")

owrite.csv( resSigarrow, file="resarrow_sig.csv")

1 write.csv(resSigarrowdown, file="arrow_down.csv")

82 write.csv(resSigarrowup, file="arrow_up.csv")

\section{C.1.2 Miscellaneous}

The following scripts contain the GO annotations, modified MA-plots from DESeq, Venn diagrams and a barplot example used in cuticle analysis.

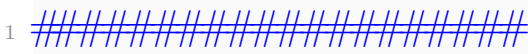

2\# GO annotations \#\#

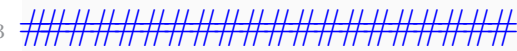

\#\#read in association table

Tc_GO $<-$ read.csv (file=" TCau2association.csv", header=T, f i ll $=\mathrm{T}$ )

7 \#\#add FB and $\mathrm{BB}$ links

${ }_{8} \mathrm{fb} . \mathrm{FBlink}=$ paste ('=HYPERLINK(" http://www.flybase.org/ reports / ',Tc_GO\$FBgnID, '. html") )', sep=' ')

9 $\mathrm{Tc} \_\mathrm{GO}<-$ cbind $\left(\mathrm{Tc} \_\mathrm{GO}\right.$, fb. FBlink $)$

10

\#\#\#\#remove NA rows, merge to Dm ortho/hom GO annotations 
12 resSigarrowdown _NA $<-$ resSigarrowdown [ complete. cases ( resSigarrowdown ),]

13 resSigarrowdown_GO <- merge (resSigarrowdown_NA, Tc_GO, by . $\mathrm{x}=$ " id" , by $\cdot \mathrm{y}=$ " au2ID" , all=F)

14 write.csv(resSigarrowdown_GO, file=" resSigarrowdown_GO.csv $"$ )

resSigarrowup_NA $<-$ resSigarrowup [ complete. cases ( resSigarrowup ), ]

resSigarrowup _GO <- merge (resSigarrowup _NA, Tc_GO, by . $x=$ =" id" , by $\cdot y="$ au2ID" , all=F)

write.csv (resSigarrowup_GO, file="resSigarrowup _GO.csv" )

\#\#\#\#\#\#\#\#\#\#\#\#|\#

\#\# MA-plots \#\#

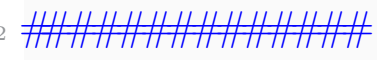

24 \#\# rename headers for plotMA function

25 resarrow $2<-$ resarrow

26 colnames $($ resarrow 2$)=\mathrm{c}("$ id" , "baseMean", "baseMeanA" , " baseMeanB", "foldChange", "log2FoldChange" , "pval" , " $\operatorname{padj} ")$

27

28 \#\#\#modified plotMA function from DESeq package

29 plotMAnew $<-$ function $(x$, ylim, col $=$ ifelse $(x \$$ padj $<=0.1$

$\& \operatorname{abs}(x \$ \log 2$ FoldChange $)>=1$, "red3", "gray32"),

linecol = "\#ff000080", xlab = NULL,

ylab $=$ NULL, $\log =" x ", \quad$ cex $=0.45$,

$\ldots)$

33

if (! (is . data.frame (x) \&\& all (c ("baseMean", "

$\log 2$ FoldChange") \%in\%

colnames (x)))) 


\section{SCRIPTS}

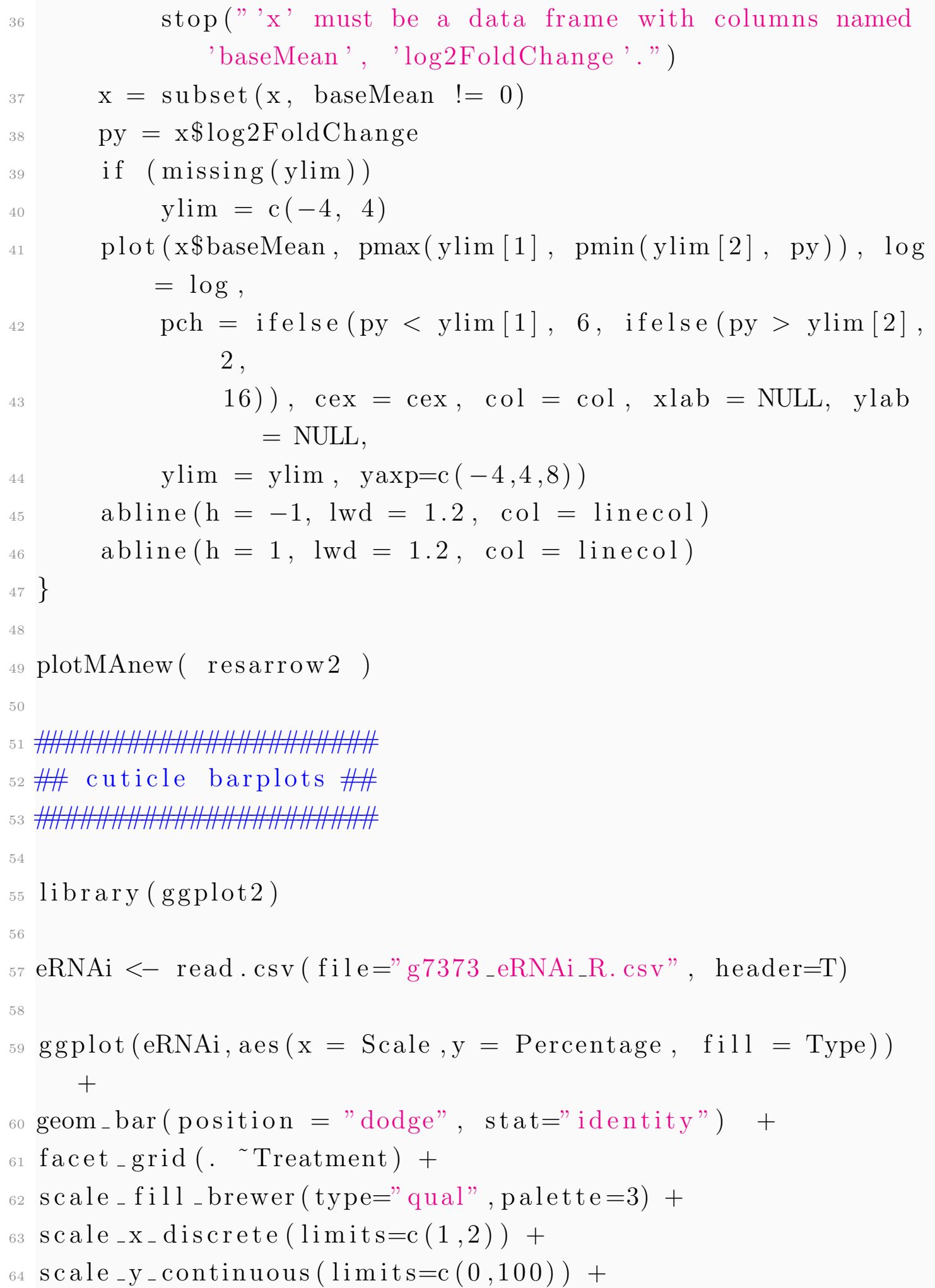


65 xlab ("injection timepoint") +

66 ylab ("Percentage")

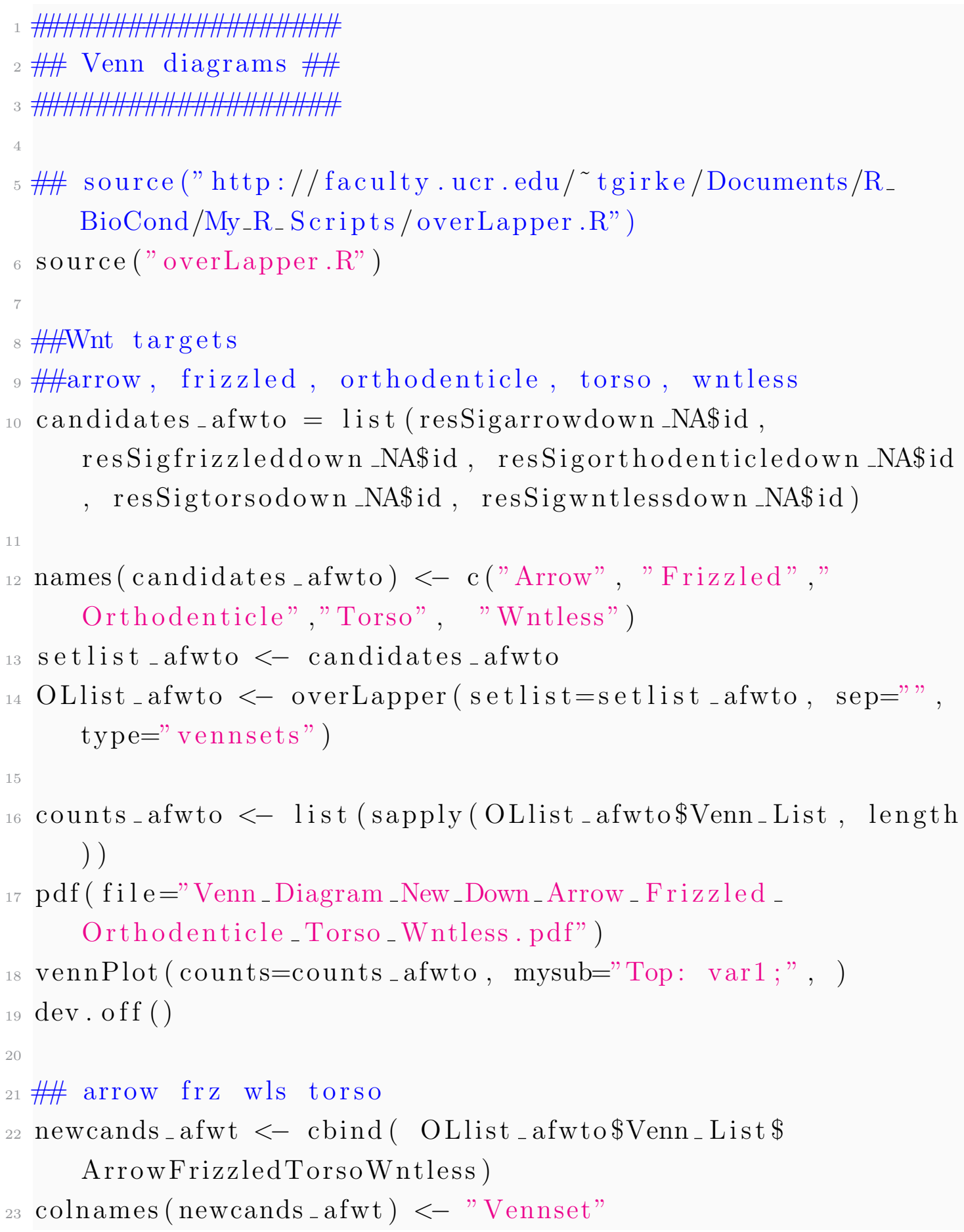




\section{SCRIPTS}

24 newcands _afwt_a $<-$ merge ( newcands_afwt, resSigarrowdown GO, by $\cdot x="$ Vennset" , by $\cdot y="$ id" , all=F)

25 write.csv (newcands_afwt_a, file="Venn_New_Down_Arrow _

Frizzled_Wntless_Torso.csv")

26

${ }_{27}$ \#\# arrow frz wls otd

28 newcands_afwo $<-$ cbind $($ OLlist_afwto $\$$ Venn_List $\$$

ArrowFrizzledOrthodenticleWntless)

29 colnames (newcands_afwo ) <- "Vennset"

newcands_afwo _a $<-$ merge ( newcands_afwo, resSigarrowdown

GO, by $\cdot \mathrm{x}=$ "Vennset", by $\cdot \mathrm{y}=$ "id", all=F)

write.csv (newcands_afwo_a, file="Venn_New_Down_Arrow_

Frizzled_Wntless_Orthodenticle.csv")

32

34 \#\#\#HH targets

5 \#\# hedgehog, torso, orthodenticle

candidates_hto $=$ list (resSighedgehogdown_NA $\$$ id , resSigorthodenticledown_NA $\$$ id ，resSigtorsodown_NA $\$$ id )

37

3 names (candidates_hto) <- c ( "Hedgehog", "Orthodenticle" , "Torso")

setlist_hto $<-$ candidates_hto

OLlist_hto $<-$ overLapper ( setlist=setlist_hto, sep=" , type $="$ vennsets")

counts_hto $<-$ list (sapply (OLlist_hto $\$$ Venn_List, length))

$\operatorname{pdf}(\mathrm{file}=$ "Venn_New_Down_Hedgehog_Orthodenticle_Torso.pdf")

vennPlot ( counts=counts_hto, mysub="Top: var1;")

dev. off ()

7 \#hedgehog torso

cands_hto $<-$ cbind ( OLlist_hto $\$$ Venn_List $\$$ HedgehogTorso)

colnames $($ cands_hto $)<-$ "Vennset" 
50 cands_hto_a $<-$ merge ( cands_hto, resSighedgehogdown_GO, by . $\mathrm{x}=$ "Vennset" , by $\cdot \mathrm{y}=$ " id" , all=F)

51 write.csv (cands_hto_a, file="Venn_Down_New_Hedgehog_Torso . csv")

52

53 \#\# hedgehog orthodenticle

${ }_{54}$ cands_ho $<-$ cbind $($ OLlist_hto $\$$ Venn_List $\$$

HedgehogOrthodenticle )

55 colnames (cands_ ho) $<-$ "Vennset"

${ }_{56}$ cands_ho_a $<-$ merge ( cands_ho, resSighedgehogdown_GO, by.x $="$ Vennset", by $\cdot \mathrm{y}=$ "id", all=F)

57 write.csv (cands_ho_a, file="Venn_Down_New_Hedgehog

Orthodenticle.csv") 


\section{SCRIPTS}

\section{C.2 Console}

\section{C.2.1 Mapping and counting RNAseq reads}

This script contains the mapping parameters used within bowtie2 and the steps in samtools to get the final counts and mapping statistics.

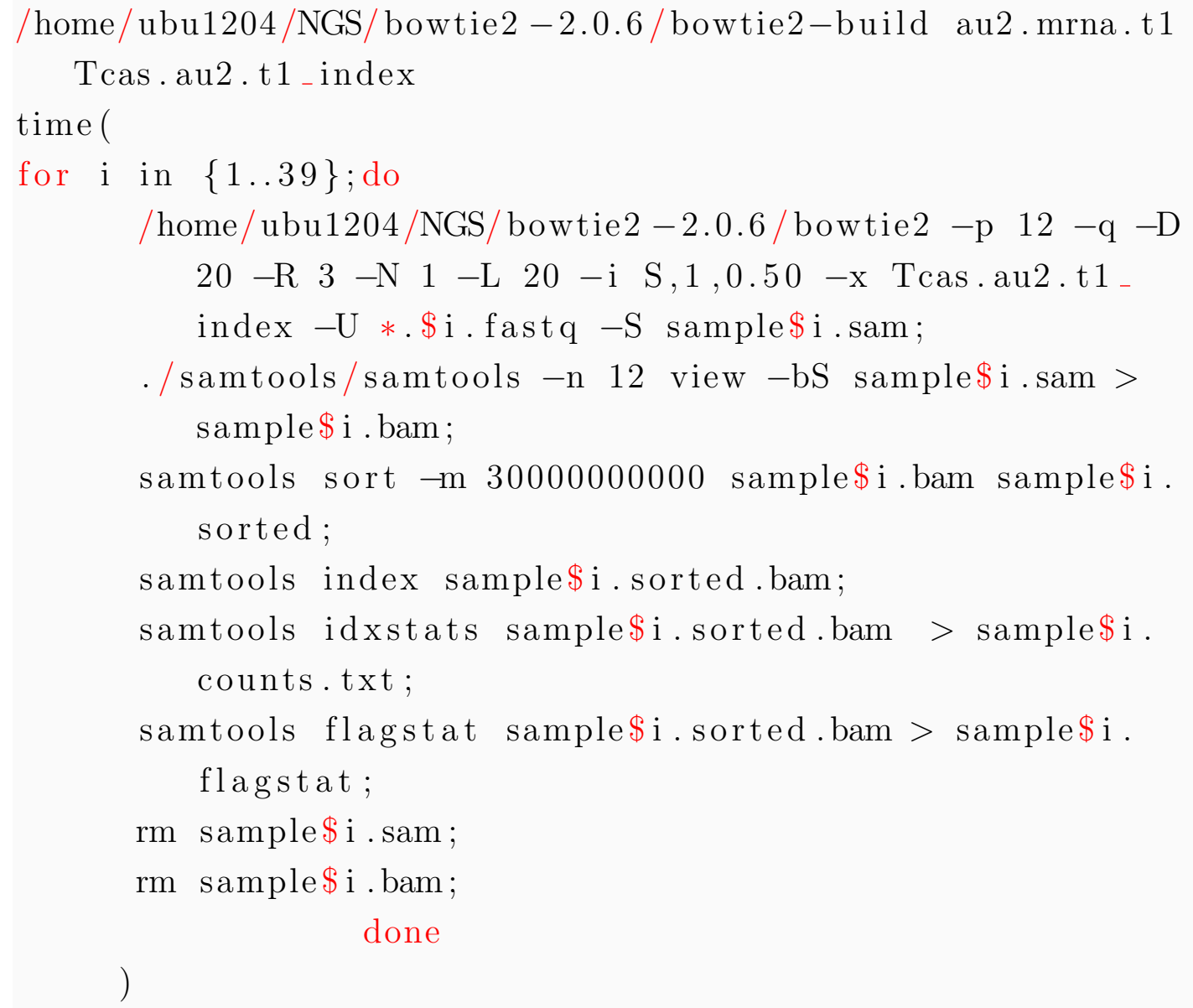

\section{C.2.2 BLAST}

This is an example on how to use BLAST. First the reference database is formatted. Then the query sequences, stored in a fasta file, can be BLASTed. Blastn was used to check the sequences of clones. Blastp was used to identify homologous sequences in other species. 


\section{C.2 Console}

1 \# protein blast, reference is a fasta file with amino acid sequences

formatdb -i reference_amino_acid.fasta $-\mathrm{o} T-\mathrm{p} T$

\# -b, number of alignments shown -d, reference - i query sequences

4 blastall - p blastp - b $1-$ d reference.fasta $-i$ query.fasta -o output_file

6 \#nucleotide blast, reference is a fasta file with nucleotide sequences

formatdb -i reference_nucleotide.fasta $-\mathrm{p} F$

blastall - p blastn - b $1-$ d reference.fasta - i query.fasta -o output_file 
C. SCRIPTS 


\title{
Curriculum Vitae
}

\author{
Georg Oberhofer
}

\section{Address}

Petrikirchstrasse 12

37077 Göttingen

Germany

Email: goberho@gwdg.de

\section{Personal Details}

Gender: Male

Date of birth: 22nd of April, 1981

Place of birth: Innichen, Italy

Present Citizenship: Italian

\section{Education}

1987 - 1992 Elementary school, Innichen

1992 - 1995 Grammar school, Innichen

1995 - 2000 Highschool, Bruneck

07/2000 Graduation from high school in "Technical Engineering"

10/2000 Studies of chemistry, University of Innsbruck 
03/2004 Studies of chemistry, University of Vienna, specialisation part with focus on biochemistry and inorganic chemistry

05/2009 Diploma thesis at the department of biochemistry in Vienna

01/2010 PhD thesis at the department of developmental biology in Göttingen

\section{Language Knowledge}

German

Italian

English 


\section{List of Publications}

J. Fu, N. Posnien, R. Bolognesi, T. D. Fischer, P. Rayl, G. Oberhofer, P. Kitzmann, S. J. Brown, and G. Bucher. Asymmetrically expressed axin required for anterior development in tribolium. Proceedings of the National Academy of Sciences of the United States of America, 109(20):77827786, May 2012. ISSN 1091-6490. doi:10.1073/pnas.1116641109. PMID: 22552230.

A. D. Peel, J. Schanda, D. Grossmann, F. Ruge, G. Oberhofer, A. F. Gilles, J. B. Schinko, M. Klingler, and G. Bucher. Tc-knirps plays different roles in the speci- fication of antennal and mandibular parasegment boundaries and is regulated by a pair-rule gene in the beetle tribolium castaneum. BMC developmental biology, 13: 25, 2013. ISSN 1471-213X. doi: 10.1186/1471-213X-13-25. PMID: 23777260 .

K. A. Panfilio, G. Oberhofer, and S. Roth. High plasticity in epithelial morphogenesis during insect dorsal closure. Biology open, 2(11):11081118, 2013. ISSN 2046-6390. doi: 10.1242/bio.20136072. PMID: 24244847.

G.Oberhofer, D. Grossmann, T. Beissbarth and G. Bucher. RNAseq after RNAi reveals differences in anterior and posterior target genes in Tribolium. (in preparation). 